\title{
NONLINEAR MODELING OF A
}

\section{SUSTAINABLE MATERIAL}

\author{
A Thesis \\ presented to \\ the Faculty of California Polytechnic State University, \\ San Luis Obispo
}

In Partial Fulfillment

of the Requirements for the Degree

Master of Science in Architecture with a Specialization in Architectural Engineering

by

Jorien G. Baza

December 2010 


\section{(C) 2010 \\ Jorien G. Baza \\ ALL RIGHTS RESERVED}




\section{COMMITTEE MEMBERSHIP}

TITLE:

AUTHOR:

DATE SUBMITTED: December 2010

COMMITTEE CHAIR: $\quad$ Edmond Saliklis, Ph.D., P.E.

COMMITTEE MEMBER: Kevin Dong, P.E., S.E.

COMMITTEE MEMBER: Mary Forte, M.A. 


\section{ABSTRACT \\ Nonlinear Modeling of a Sustainable Material \\ Jorien G. Baza}

This study developed a nonlinear constitutive model for a sustainable orthotropic material. Existing methods for constitutive models of wood were improved upon to include the nonlinear stress-strain response not only in the two orthogonal axes but any orientation to the strong axis of this material. This method also simplifies the nonlinear stress-strain relationships into bilinear stress-strain curves which can be valuable in hand calculations as well as finite-element analyses. The effectiveness of the proposed constitutive model is demonstrated by comparing bilinear stress-strain predictions to experimental data. 


\section{TABLE OF CONTENTS}

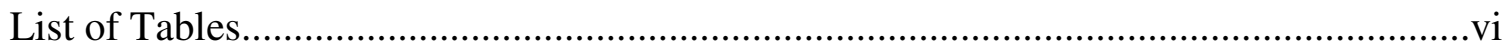

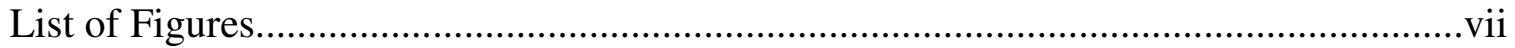

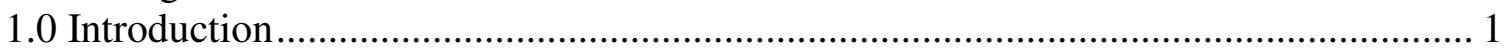

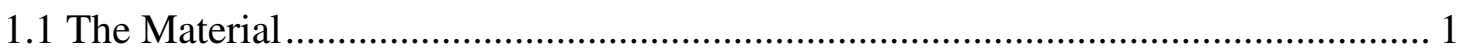

1.2 The Experimental Testing ............................................................................ 3

1.3 The Mathematical Model ............................................................................ 3

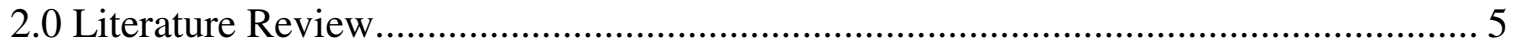

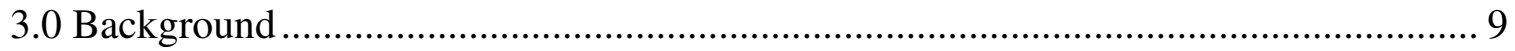

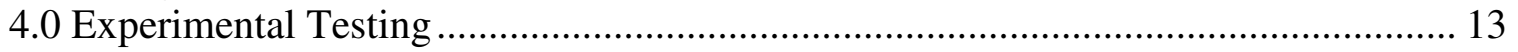

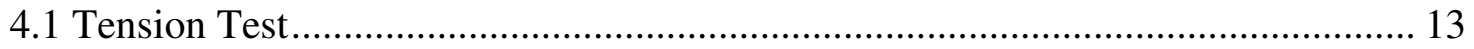

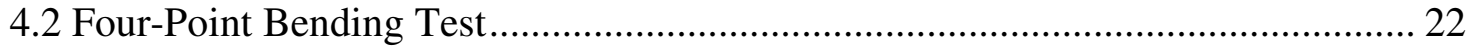

4.3 Simulated Four-Point Bending Test.................................................................. 32

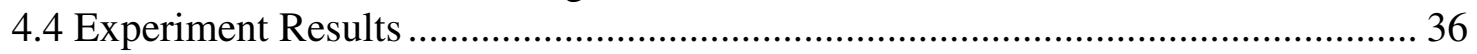

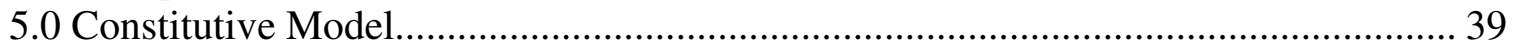

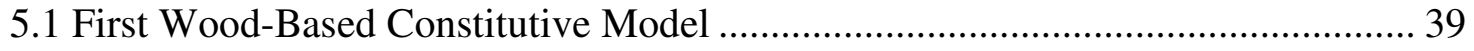

5.2 Second Wood-Based Constitutive Model........................................................... 41

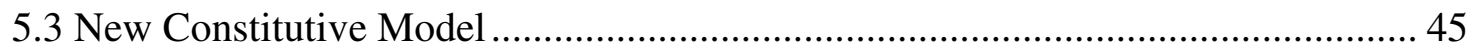

6.0 Application in Finite Element Analysis .............................................................. 53

6.1 Computer Modeling .................................................................................... 53

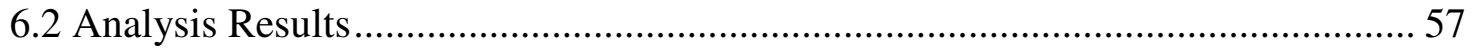

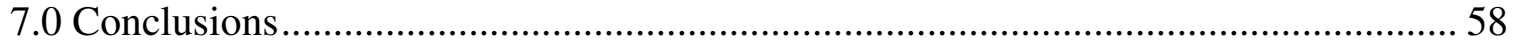

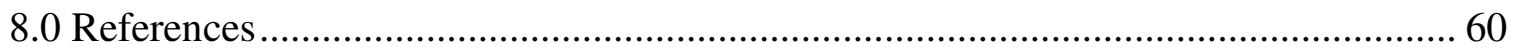

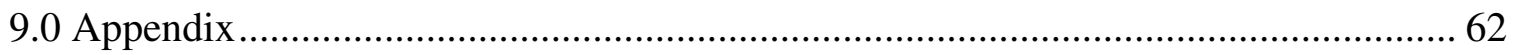

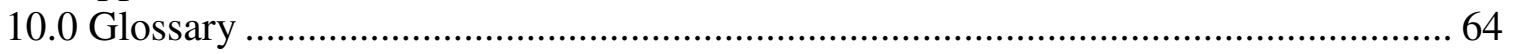

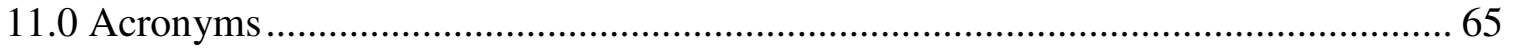




\section{LIST OF TABLES}

Table 1: Flexural Strength (Modulus of Rupture) for Wood and Composite................... 5

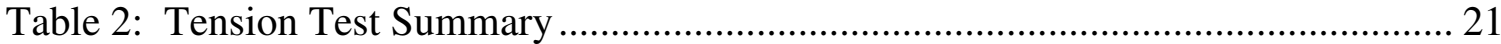

Table 3: 4pt Bending Experiment Specimen Summary .............................................. 30

Table 4: 4pt Bending Simulated Experiment Specimen Summary ............................... 34 


\section{LIST OF FIGURES}

Figure A: PP/kenaf Extrusion Process ........................................................................ 2

Figure B: Elastic Modulus with Respect to UV Exposure Hours...................................... 9

Figure C: Sample Compression Specimen ………………….................................... 10

Figure D: HDPE/Fiber Compression Tests................................................................ 11

Figure E: Similar Material Stress-Strain Curve with Bilinear Approximation................. 12

Figure F: Labeled Axes of a Board.................................................................... 14

Figure G: Tension Test Specimens ....................................................................... 15

Figure H: Measuring Test Specimen Dimensions ………………………………….... 16

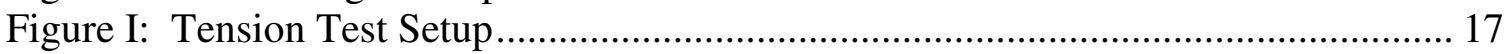

Figure J: Desirable Failure Mechanism ............................................................... 18

Figure K: Undesirable Failure Mechanism............................................................. 18

Figure L: Tension Specimen after Failure …………............................................... 19

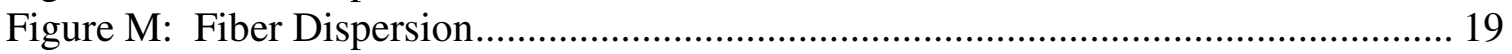

Figure N: Sample Tension Test Stress-Strain Curve ……………………………….... 20

Figure O: Initial Linear Young's Modulus Summary ………….................................... 21

Figure P: Nonlinear Young's Modulus Summary ........................................................ 22

Figure Q: Four-Point Bending Test Setup ……………………………………….... 23

Figure R: Shear and Moment Diagrams for 4-Point Bending Test ................................. 24

Figure S: Four-Point Bending Test Setup ............................................................... 26

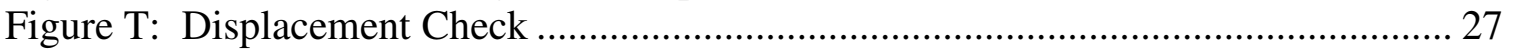

Figure U: Universal Testing Machine Readouts........................................................ 28

Figure V: Cross-Section of Unplaned Board ........................................................... 29

Figure W: Sample Plot of $\delta$ vs. $P l^{3} / 432 I$, Eq. 6, for a Specimen .................................... 30

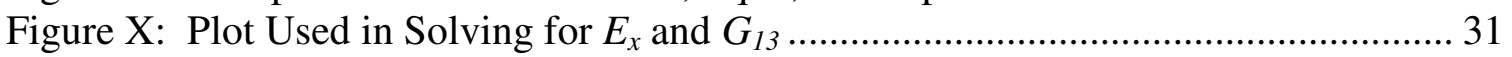

Figure Y: Simulated Experiment Test Setup and Beam Mesh ...................................... 33

Figure Z: Plot Used in Solving for $E_{x}$ and $G$ for Simulated 4pb Experiment $(s=1.2) \ldots 34$

Figure AA: Plot Used in Solving for $E_{x}$ and $G$ for Simulated $4 \mathrm{pb}$ Experiment $(s=1.5) 35$

Figure BB: Fitting Eq. 8 to Experimental Data ……………........................................ 37

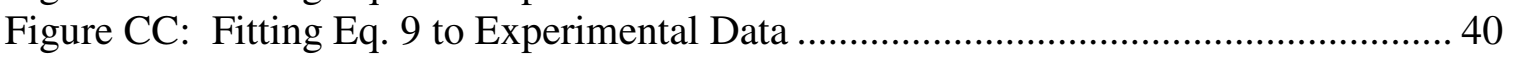

Figure DD: Fitting Eq. 10 to Experimental Data........................................................ 41

Figure EE: Bilinear Constitutive Model ............................................................ 42

Figure FF: Fitting a Bilinear Curve to the 1-Axis......................................................... 44

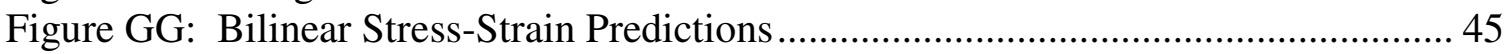

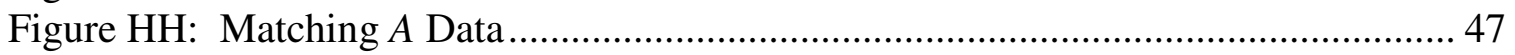

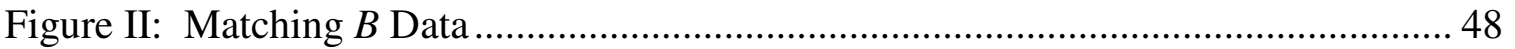

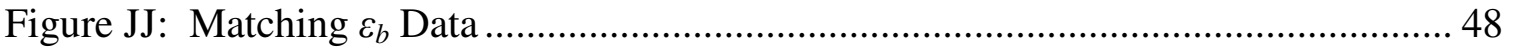

Figure KK: Bilinear Prediction for $33^{\circ}$ Data ........................................................... 50

Figure LL: Bilinear Prediction for $45^{\circ}$ Data ........................................................... 51

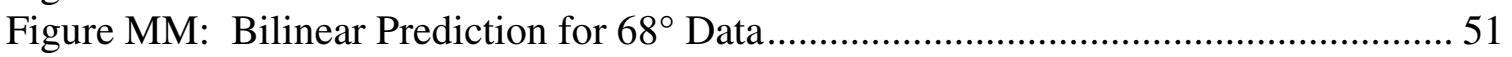

Figure NN: Summary of Bilinear Stress-Strain Predictions ........................................... 52

Figure OO: Analyzed Frame.............................................................................. 53

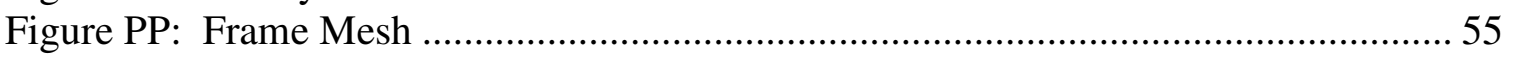

Figure QQ: Close-up of Element Local Axes................................................................ 56

Figure RR: Force-Displacement Relationship for the Analyzed Frame........................... 57 


\section{LIST OF NOMENCLATURE}

A Cross-sectional area

E Young's modulus

$E_{\text {linear }}$ Young's modulus in the linear range

$E_{\text {nonlin }}$ Young's modulus in the nonlinear range

$E_{s} \quad$ Apparent Young's modulus

$E_{x} \quad$ Young's modulus in the $0^{\circ}$ axis

$G \quad$ Shear modulus

I Moment of inertia of the cross-section

$l \quad$ Length of the specimen

MOR Modulus of rupture

$P \quad$ Applied load

$\alpha \quad$ Constant used in calculating bilinear point strain

$\gamma \quad$ Shear strain

$\delta \quad$ Maximum vertical deflection

$\delta_{b} \quad$ Vertical deflection due to bending

$\delta_{s} \quad$ Vertical deflection due to shear

$\varepsilon \quad$ Strain

$\sigma \quad$ Stress

$\tau \quad$ Shear stress

$v \quad$ Poisson's ratio

$h / l \quad$ Depth/Span ratio 


\subsection{INTRODUCTION}

This thesis is part of a larger research project called The Emergency Shelter Project. The Emergency Shelter Project is being developed by the Department of Architectural Engineering, the Department of Architecture, and the Department of Materials Engineering at California Polytechnic State University, San Luis Obispo. The overall goal of the larger project is to design a temporary shelter that is durable, economical, rapidly assembled, and made out of a sustainable material. The purpose of this thesis was to find the material's stress-strain properties for use in nonlinear analyses. These properties were found experimentally and then further explored via finite element analyses. The gathered data was used for the development of a new semi-empirical constitutive model.

\subsection{The Material}

The sustainable material that was used is a $65 \%$ plastic and $35 \%$ fiber composite. In this study, the plastic was a polypropylene (PP) homopolymer, but a high-density polyethylene (HDPE) has also been used. Polypropylene can be recycled from used plastic products such as grocery bags and milk jugs. The fibers are $\approx 1 \mathrm{~mm}$ long kenaf fibers and are used as reinforcement within the recycled plastic matrix. Kenaf is a plant that is not normally used in current wood/plastic products. The renewable resources for the fibers and the plastic matrix make this material sustainable. The larger Emergency Shelter Project team investigated the use of this material to create rapidly assembled shelters. 
The plastic is mixed with the organic fibers and is extruded out of a machine as shown in Figure A.

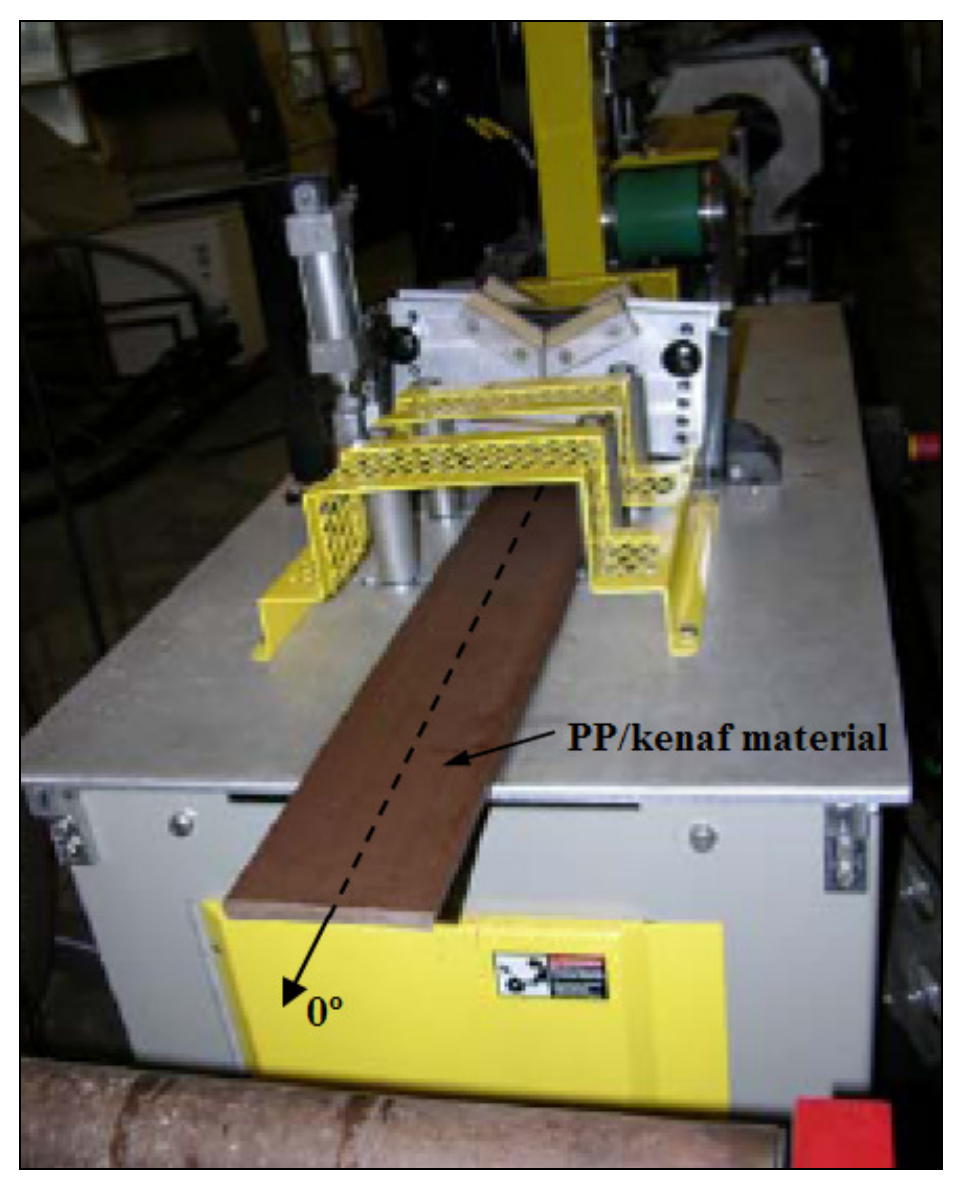

Figure A: PP/kenaf Extrusion Process

Source: Saliklis 2009

Usually, the PP/kenaf material is extruded into planks, but it can also be injection molded into virtually any shape or size. Both processes align most of the fibers along the long or longitudinal axes, creating an orthotropic ${ }^{1}$ material similar to wood since the other axes have fewer aligned fibers. All test specimens in this study came from extruded planks

${ }^{1}$ Key terms in bold are defined in the glossary 
with the dimensions of 5.125" wide, 1.125 " thick, and 48" long. Throughout this study, the $0^{\circ}$ axis will refer to this longitudinal extrusion axis.

\subsection{The Experimental Testing}

Experiments were conducted on this material to find the shear modulus $G$ and Young's modulus $E$ at varying angles to the strong axis in both the linear-elastic range and nonlinear range. Fifty-six tension tests were performed to solve for Young's modulus, and nine four-point bending tests were performed to solve for the shear modulus. These experimentally obtained values formed the basis of the body of data used to create a new constitutive model.

\subsection{The Mathematical Model}

The proposed constitutive model developed for this material is an extension of two previously derived constitutive models for orthotropic wood based materials. The first wood-based model, derived by Saliklis and Falk (2000), predicts the shear modulus and Young's modulus at varying angles to the strong axis in an orthotropic material. The second model, derived by Saliklis, Urbanik, and Tokyay (2003), predicts the shear modulus and Young's modulus in the nonlinear range in the strong axis and at ninety degrees to the strong axis. A weakness of the first model is that it applies only to the linear region. A weakness of the second model is that it does not capture any response other than $0^{\circ}$ and $90^{\circ}$. The newly proposed constitutive model is a combination of the two wood-based models as it predicts both a linear and nonlinear Young's modulus at any orientation to the strong axis. The advantage of this model is the application of a simple bilinear stress-strain relationship for a clearly nonlinear material. A bilinear 
stress-strain curve simplifies hand analyses and may be necessary when inputting material properties into many structural analysis programs. This model's ease of implementation will be demonstrated by a nonlinear finite element analysis (FEA) of a lateral load resisting frame. A weakness of the new model is that it does not mechanistically link the shear response to the axial response as did the previous two models. Further experimental testing would be required to create such a mechanistic linkage. 


\subsection{LITERATURE REVIEW}

A growing focus of the building industry is to create "greener" buildings. In addition to designers creating better energy-saving methods, greater attention is being given to the use of recyclable materials such as steel and plastics. The high-density polyethylene (HDPE) or polypropylene (PP) composites are attractive because they can be recycled from some of the enormous waste stream currently being generated.

However, further research of the design properties is necessary to give designers the confidence they need to begin using the product in structural systems.

Compared to wood, the industrial scale fabrication of the HDPE ensures a more consistent material makeup: fewer knots, less grain inclination, etc. According to Saliklis' and Arens' paper “Rapidly assembled emergency shelters made from 'green' materials" (2009), the more consistent makeup results in a lower standard deviation and comparable design values. Saliklis' and Arens' paper gives experimental modulus of rupture $M O R$ data for an HDPE material similar to that used in this study and is shown in Table 1 below.

\begin{tabular}{|c|c|c|c|c|}
\hline Material & $\begin{array}{c}\text { Modulus of Rupture } \\
\text { (MOR) }\end{array}$ & $\begin{array}{c}\text { MOR } \\
\text { coeff of variation }\end{array}$ & $\begin{array}{c}\text { MOR } \\
\text { std. deviation }\end{array}$ & Design strength * \\
\hline \hline $\begin{array}{c}\text { Loblolly Pine } \\
\text { ag. fiber/plastic composite }\end{array}$ & $\begin{array}{c}88.2 \mathrm{MPa}(12,800 \mathrm{psi}) \\
55.2 \mathrm{Mpa}(8,000 \mathrm{psi})\end{array}$ & $25 \%$ & $22.0 \mathrm{MPa}(3,200 \mathrm{psi})$ & $24.7 \mathrm{MPa}(3589 \mathrm{psi})$ \\
$2.2 \mathrm{MPa}(320 \mathrm{psi})$ & $24.5 \mathrm{MPa}(3559 \mathrm{psi})$ \\
\hline \hline
\end{tabular}

\section{Table 1: Flexural Strength (Modulus of Rupture) for Wood and Composite Source: Saliklis 2009}

Their data shows that pine has a modulus of rupture of 12,800 psi, with a standard deviation of 3,200 psi and design strength of 3589 psi. In comparison, the modulus of 
rupture data for their fiber reinforced HDPE is 8,000 psi, with a standard deviation of 320 psi and design strength of 3559 psi. The comparable design strengths of wood and HDPE make it possible to use HDPE as a structural material.

However, before HDPE can be considered in structural uses, allowable design values and material properties need to be established such as the elastic moduli and yield stresses.

Young's modulus can be experimentally derived through a number of tests including bending, compression, and tension tests. American Society for Testing and Materials (ASTM) standards prescribes known procedures to solve for Young's modulus. However, finding the shear modulus is not as straightforward. Difficulties arise in test setups and trying to obtain data in the nonlinear range. The shear modulus can be derived many different ways including bending theory, torsion tests, and pure shear tests, as stated in "A New Test Method for Measuring the Longitudinal and Shear Moduli of Fiber Reinforced Composites” by S. Javad Jalali and Farid Taheri (1999). Possible shear tests as suggested by the literature review include the following:

- ASTM 3044

- Torsion test

- Iosipescu shear test (Yoshihara 1999)

- Three-point bending test (Yoshihara 1998)

- Four-point bending test (Yoshihara 2002)

It is not certain whether the ASTM shear test could be used for this plastic-fiber composite because it is intended for wood-based panels. In addition, the ASTM test for shear cannot produce data past the linear-elastic range because the shear properties found are derived from elastic theory. Both the torsion test and Iosipescu shear test would be 
ideal since they load the member in pure shear with no bending deflection. However, the torsion test assumes the shear modulus is the same for all planes parallel to the strong axis, which is correct to assume for an isotropic material but incorrect to assume for this orthotropic material. The Iosipescu shear test consists of a complicated test setup requiring a special test fixture.

Yoshihara's article "Measurement of the shear modulus of wood by asymmetric four-point bending tests" gives a comparison among the four-point bending test, threepoint bending test, and a pure shear test. The results of Yoshihara's study show that a four-point bending test can properly solve for the shear modulus when using a shear factor of 1.5 and a specimen length at least 20 times greater than its depth (Yoshihara 2002). Using shallow beams in a shear test seems counter-intuitive; however, Yoshihara's study demonstrated that smaller depth/span ratios reduce the extra deflection caused by stress concentrations. This study also compared the use of 1.2 and 1.5 for the shear factor, and showed that a value of 1.2 overestimated the shear modulus and a value of 1.5 was more appropriate. Other research has also investigated using a shear factor of 1.5 (Bindzi and Samson 1995). Yoshihara's paper provides a relationship between the shear modulus and Young's modulus, such that both can be obtained through a least squares error minimization.

Each of Yoshihara's bending tests applies Timoshenko Beam Theory, which assumes that the deflection of a beam is composed of bending deflection and shear deflection. Because these tests rely on elemental bending theory, they are not used for obtaining data past the linear-elastic range. The inherent difficulty of obtaining sound 
nonlinear experimental shear data explains why this thesis did not attempt to mechanistically link nonlinear shear response to nonlinear axial response.

The model proposed here is an extension of the bilinear model proposed by Saliklis (2003). A key idea in this model is a distinct yield point on each of the three orthogonal axes. Shih and Lee (1978) also used this approach which allowed for different but linked yield points on the orthogonal axes. It is this criterion that ANSYS has adopted for nonlinear orthotropic materials and this was the reason for the extension of the previous constitutive models. The bilinear model works extremely well for materials that do not exhibit large nonlinearity. 


\subsection{BACKGROUND}

Part of the basis of this project is testing that has been done on earlier generations of the HDPE/PP composite material. Figure B below shows elastic modulus results from a study that used UV light to simulate aging (Carpenter 2009).

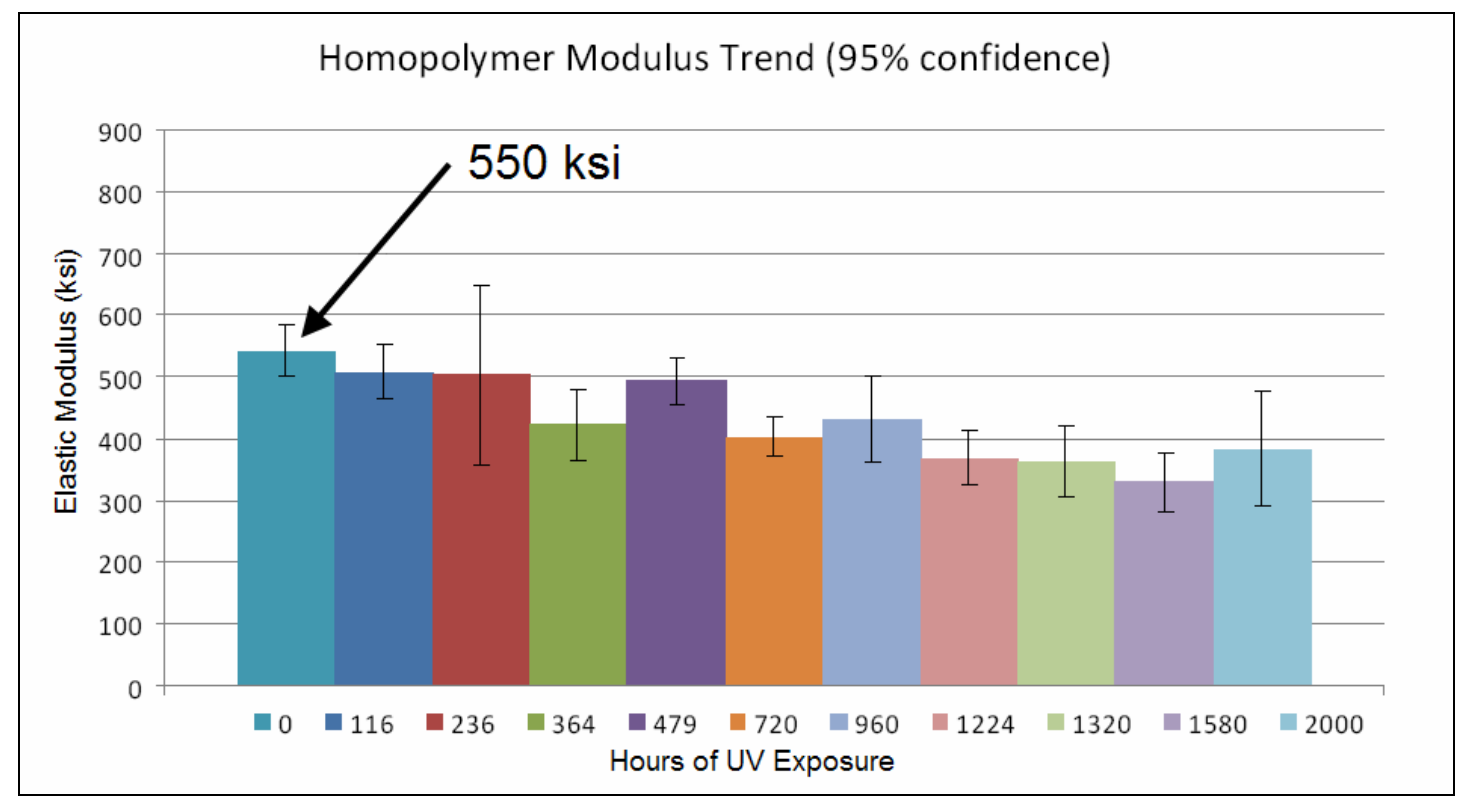

Figure B: Elastic Modulus with Respect to UV Exposure Hours Source: Saliklis 2009

The graph above shows experimentally-found elastic moduli in relation to hours of exposure to UV light. Another study that investigated the bending and compressive strength of the HDPE/PP material has shown the material to be orthotropic and very nonlinear (Lee 2007). On the next page is a sample of a compression specimen after testing, Figure C. 


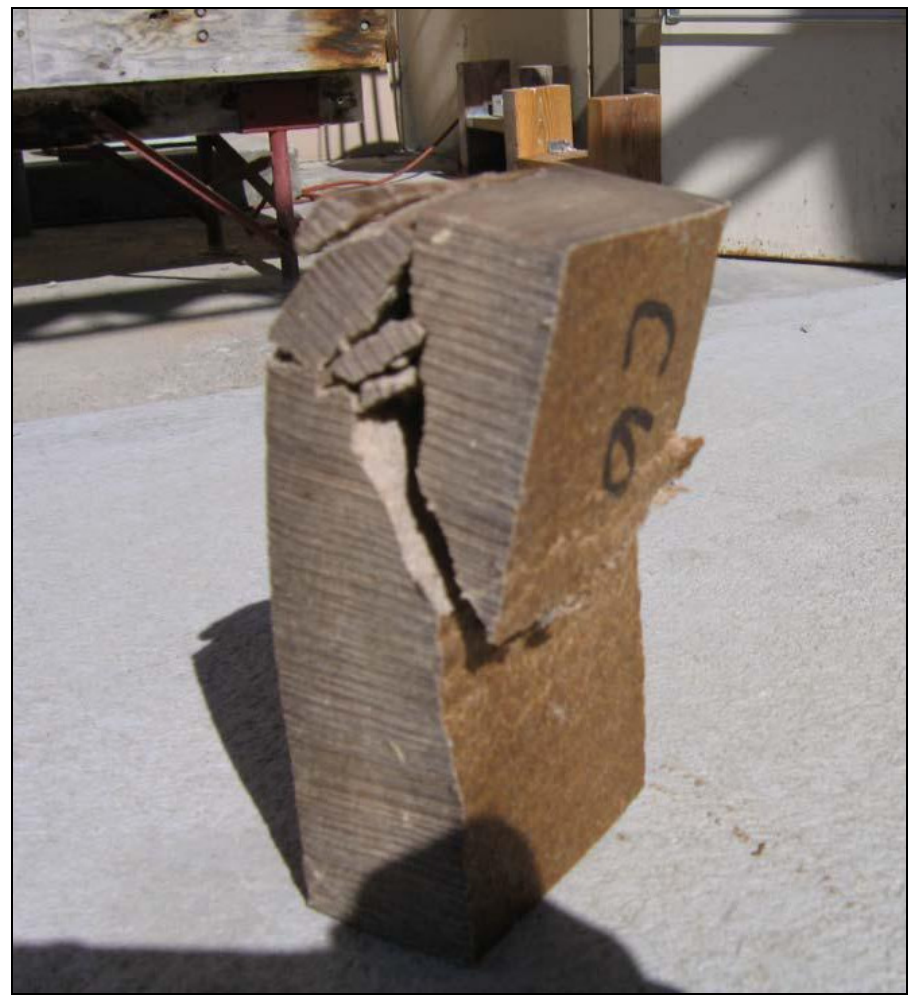

Figure C: Sample Compression Specimen

Source: Lee 2007

Figure D, on the following page, shows sample stress-strain curves for a similar HDPE material subject to compression. 


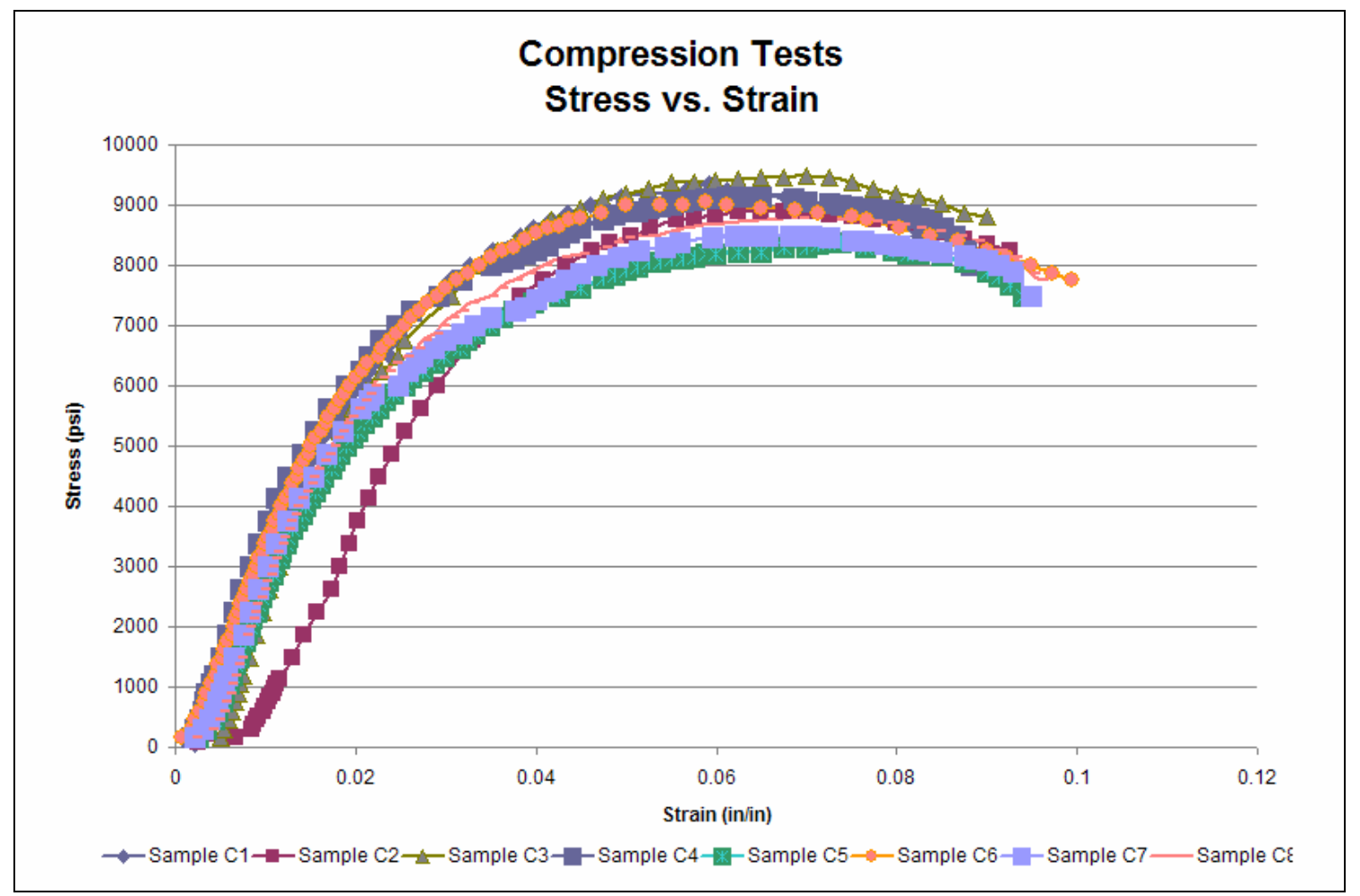

Figure D: HDPE/Fiber Compression Tests

Source: Lee 2007

The stress-strain relationship shows that the material is very non-linear. Yet even for such markedly nonlinear curves, a bilinear curve fit can still determine the initial elastic and nonlinear moduli. A sample stress strain curve with a bilinear approximation is shown in Figure E on the following page. 


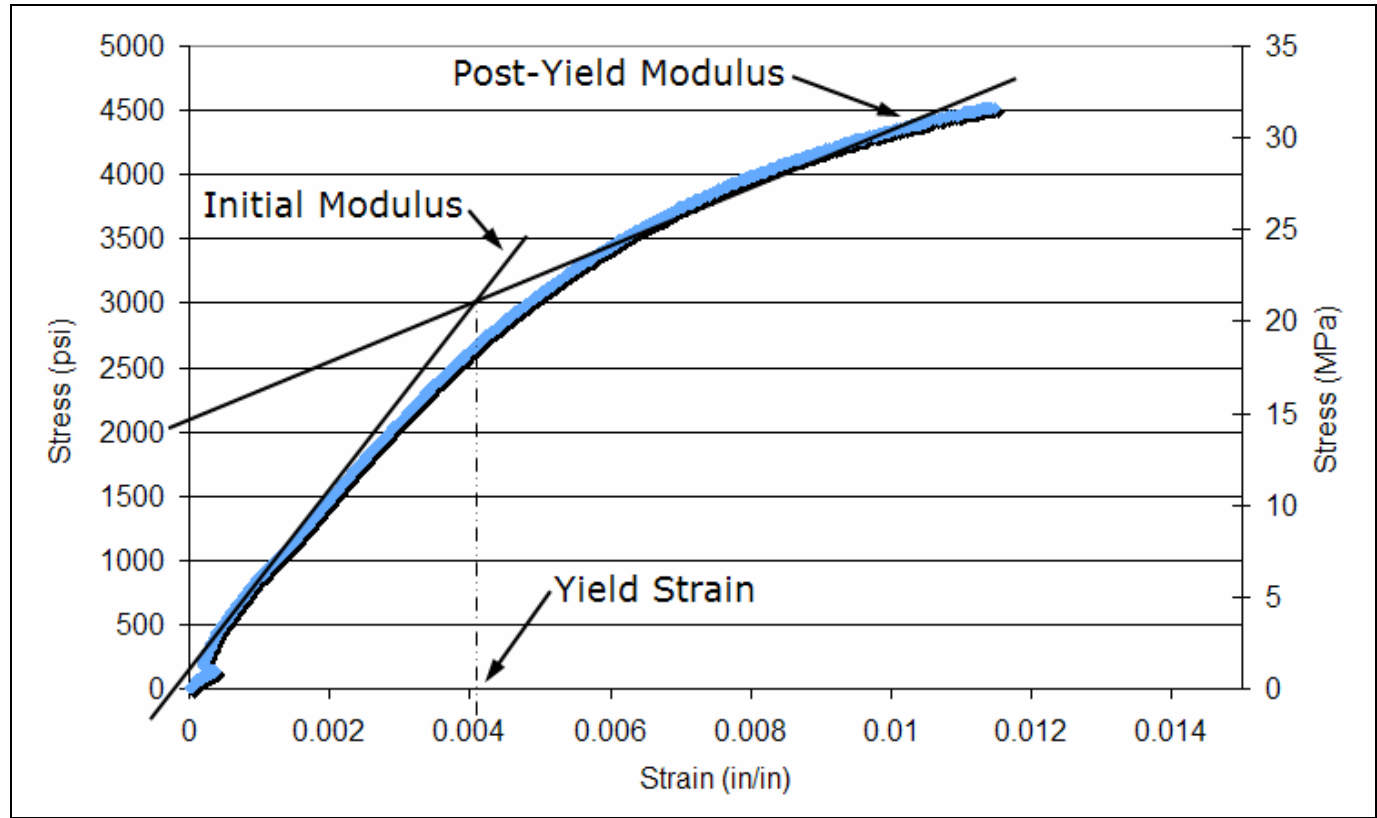

Figure E: Similar Material Stress-Strain Curve with Bilinear Approximation Source: Saliklis 2009

In the figure above, the slopes of the two curve-fitted lines represent the modulus, one in the linear and one in the nonlinear range. Literature often refers to the nonlinear range as the post-yield range for many materials, defining the point at which the stress-strain curve changes slope as the yield point. However, testing was not done to determine whether this PP/kenaf material actually yields at this apparent yield point. Therefore, this study defines the point at which the slope changes as the bilinear point and the subsequent modulus as the nonlinear modulus. 


\subsection{EXPERIMENTAL TESTING}

This section will discuss the experimental test methods used to solve for the material properties of the PP/kenaf composite material.

\subsection{Tension Test}

Pure tension tests were performed to determine Young's modulus in both the linear-elastic and nonlinear range. Young's modulus $E$ can be found using the experimental stress $\sigma$ and strain $\varepsilon$ data along with the relation known as Hooke's law, shown below.

$$
\sigma=E \varepsilon
$$

When the initial stress-strain data is fit with straight lines, the slopes of the lines can be recorded as the resulting moduli.

Thin test specimens were cut from planks in the 1-2 plane, see Figure F, at angles $\theta$ of $0^{\circ}, 33^{\circ}, 45^{\circ}, 68^{\circ}$, and $90^{\circ}$ from the 1 axis. 


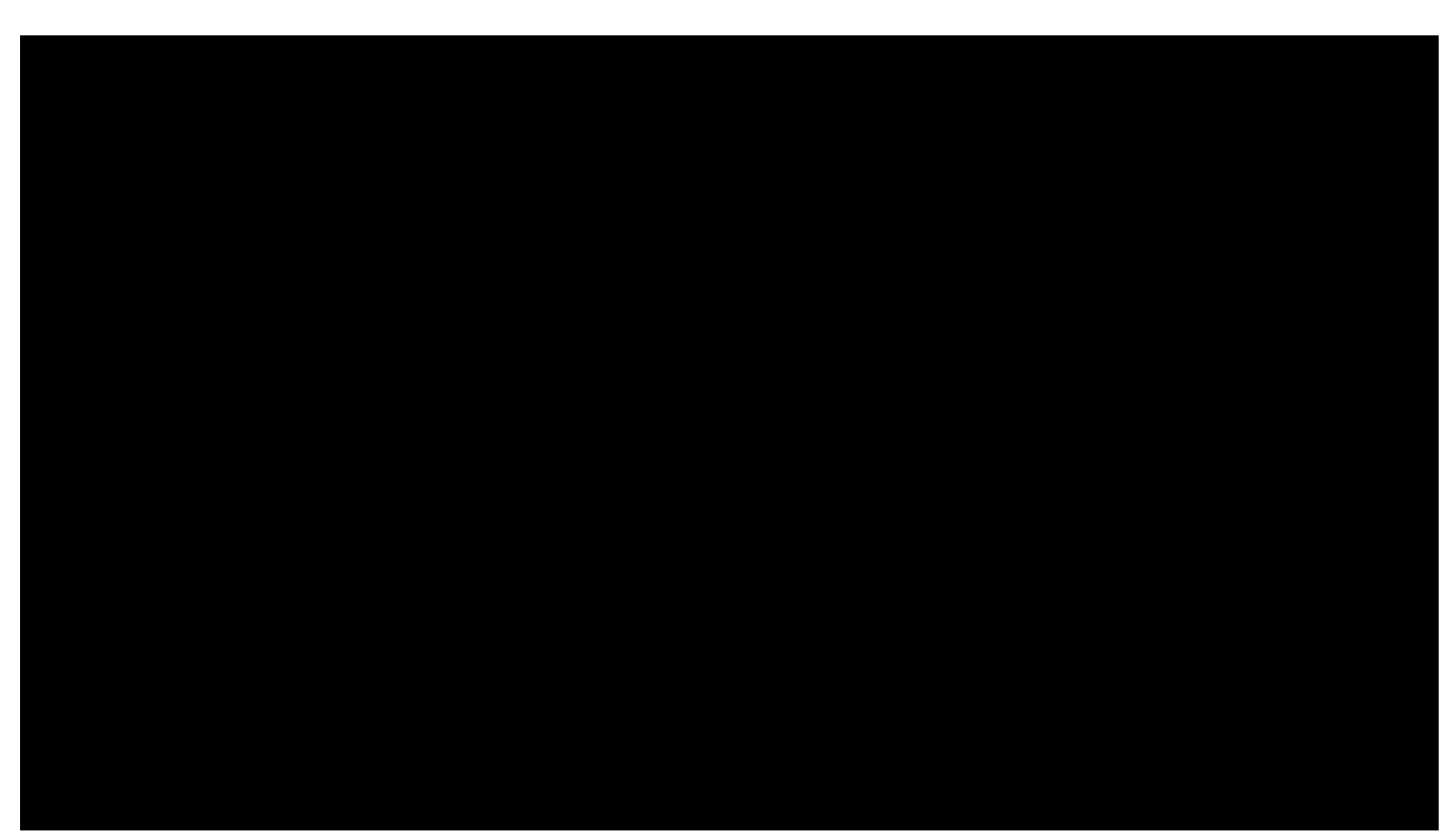

\section{Figure F: Labeled Axes of a Board}

The 12 plane was chosen because it could yield samples that were at least 5" long, or long enough to perform the tension test. Test specimens had relatively similar rectangular cross-sectional areas ranging from 0.251 to $0.396 \mathrm{in}^{2}$. The dimensions were measured using a vernier scale caliper. Samples of the specimens are shown in Figures G and $\mathrm{H}$ on the following pages. 


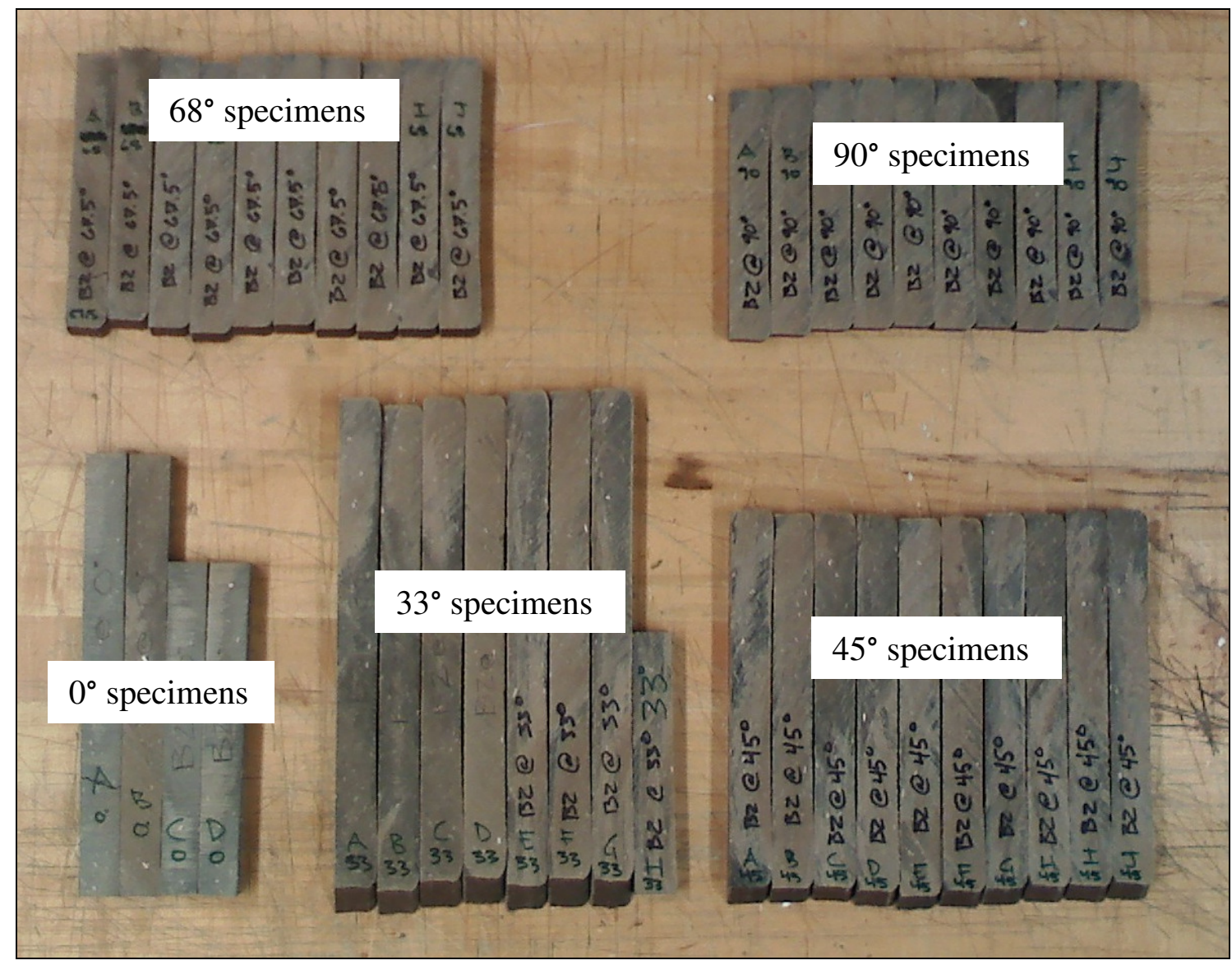

Figure G: Tension Test Specimens 


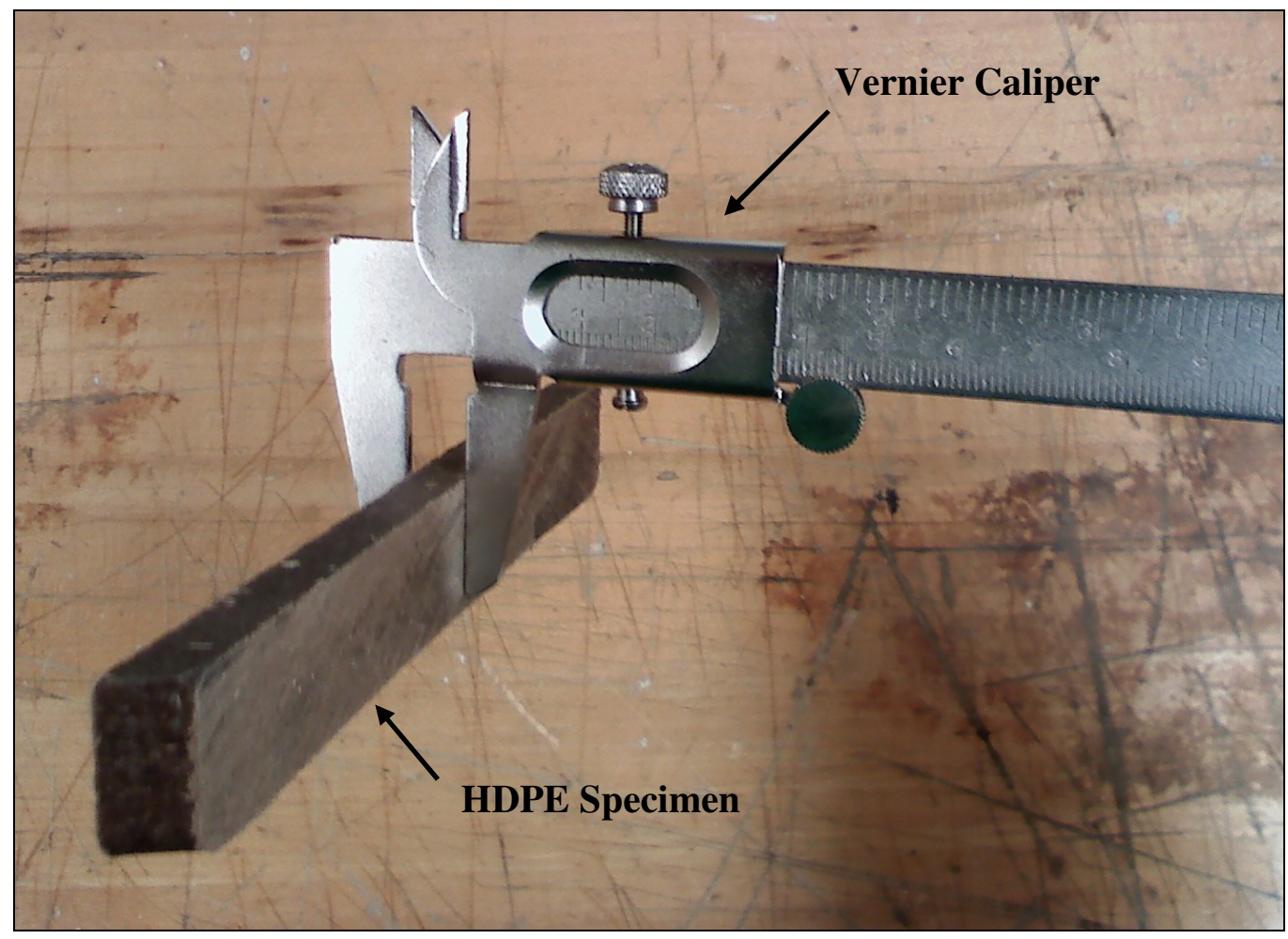

Figure H: Measuring Test Specimen Dimensions

The length and width of the original planks were $48^{\prime \prime}$ and 5.125" respectively. The $0^{\circ}$ specimens could be cut up to $48^{\prime \prime}$ long. However, the length of the specimens cut at $90^{\circ}$ could only be $5.125 "$ long, making it difficult to adequately clamp the specimen with the attached extensometer. Figure I on the following page shows the test setup. 


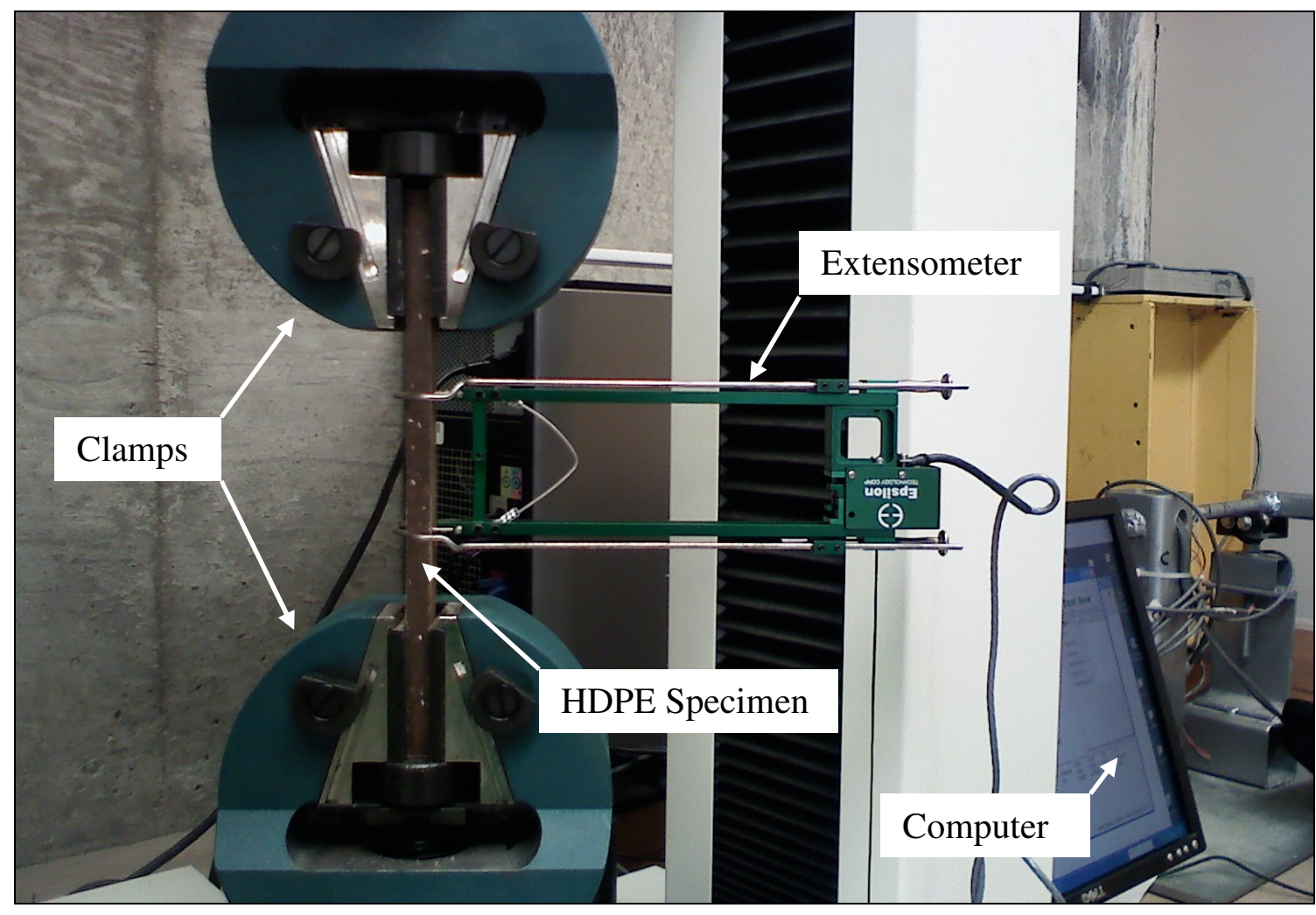

Figure I: Tension Test Setup

The Epsilon model 3542 axial extensometer was plugged into the Tinius Olsen High Force Electromechanical Tester, which is connected to the computer. The specimen was then clamped into the machine and the extensometer was connected to the specimen between the clamps. The Tinius Olsen software, along with the extensometer peripheral, recorded the tensile force and corresponding strain at each load in tabular form.

The longer specimens were easier to test because the resulting data was more consistent. Many of the shorter specimens didn't have enough material in the clamps and slipped or broke in the clamp leading to unreliable data near failure. The desirable failure mechanism is shown in Figure $\mathbf{J}$ on page 18, and the undesirable failure mechanism is shown in Figure K on page 18. 


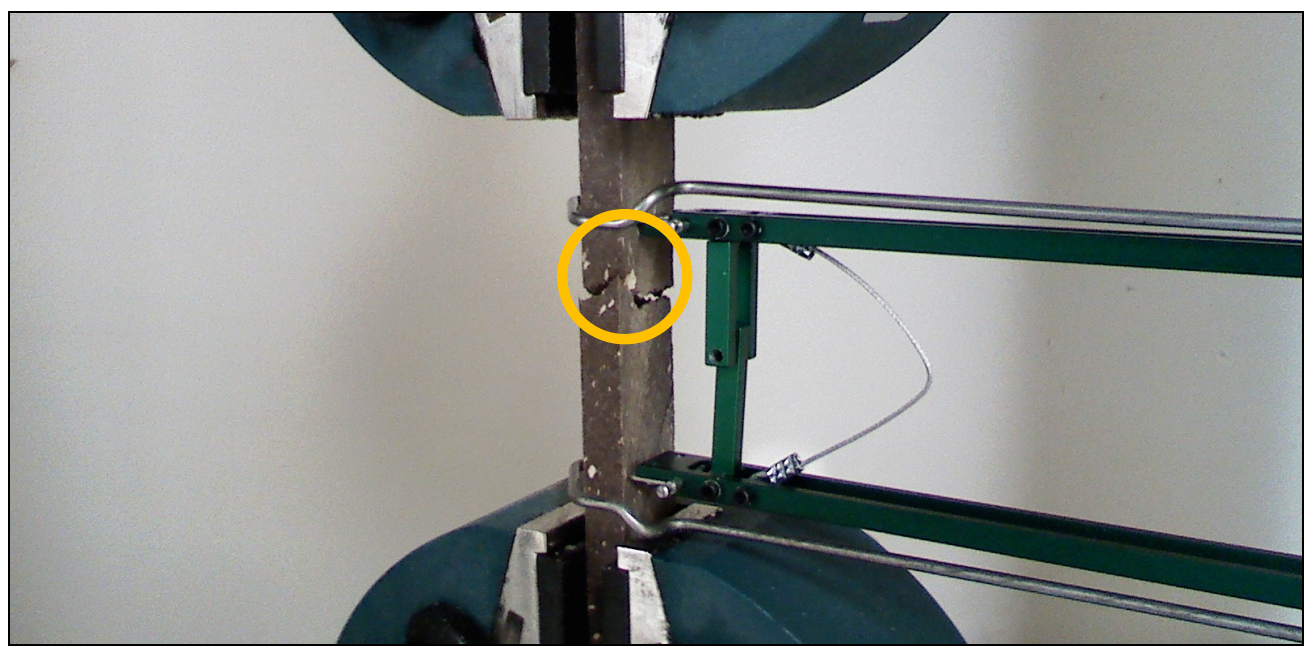

Figure J: Desirable Failure Mechanism

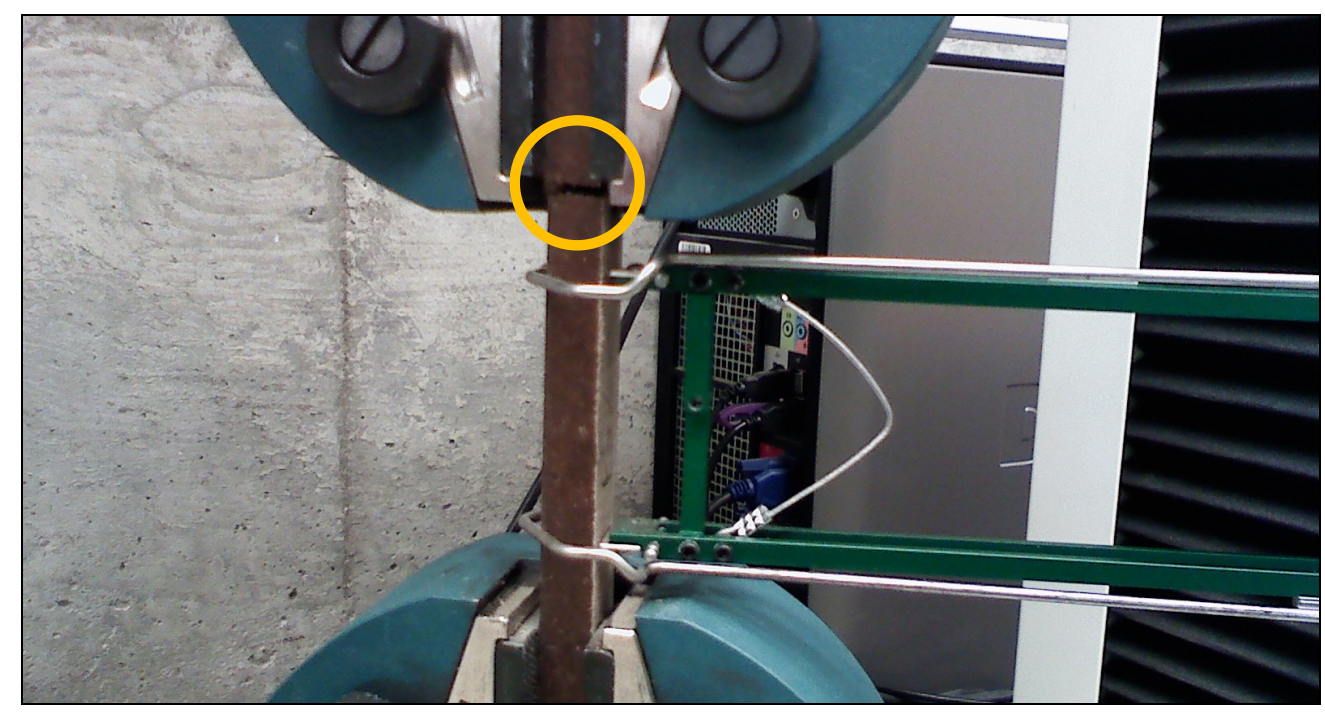

Figure K: Undesirable Failure Mechanism

Stress concentrations near the clamps led to an unreliable ultimate stress, and slippage often caused jumps in the strain data.

Another factor that affected ultimate stress was the cross-sectional area.

Specimens with smaller cross-sections failed at lower stresses than specimens with larger cross sections. A reason for this behavior may be that during production of the material, some fibers settle too close together in the matrix creating weak spots called fiber pockets. 
As a result, larger cross-sections are more uniform than smaller ones. Studies have been done on similar fiber-reinforced composites showing the relationship between fiber dispersion and tensile strength (Raj and Kotka 1991). Fiber dispersion can be clearly seen in Figures L and M below.

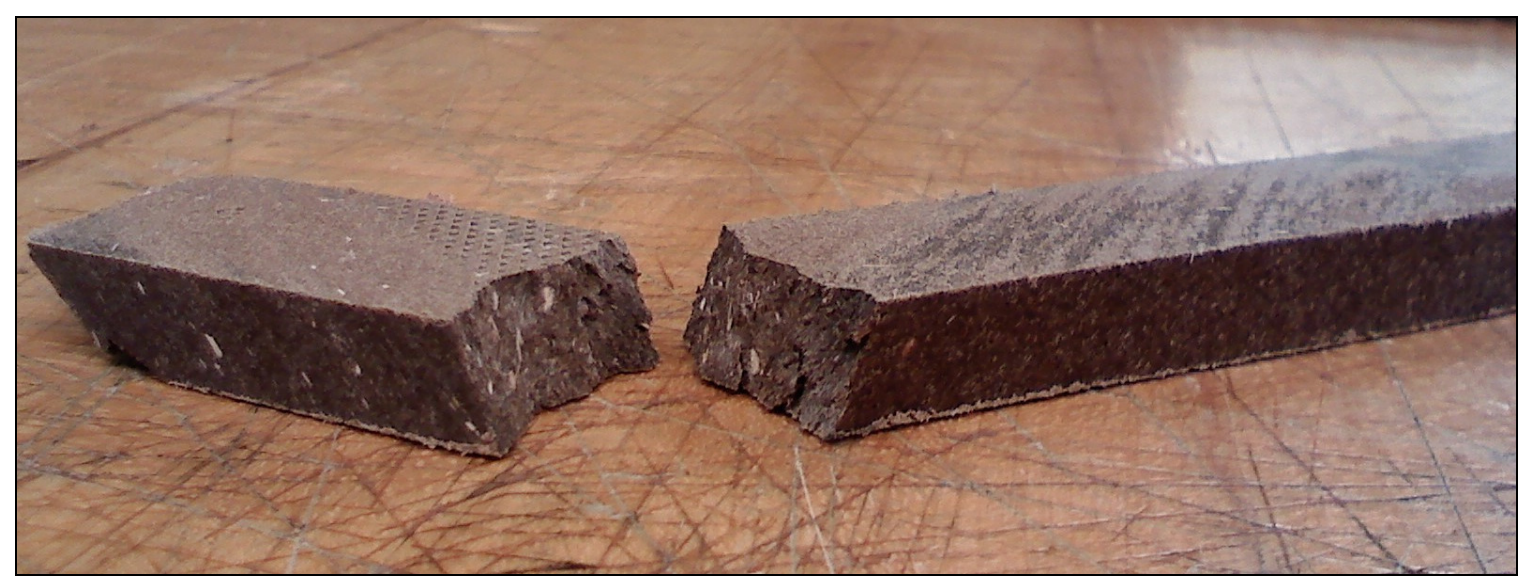

Figure L: Tension Specimen after Failure

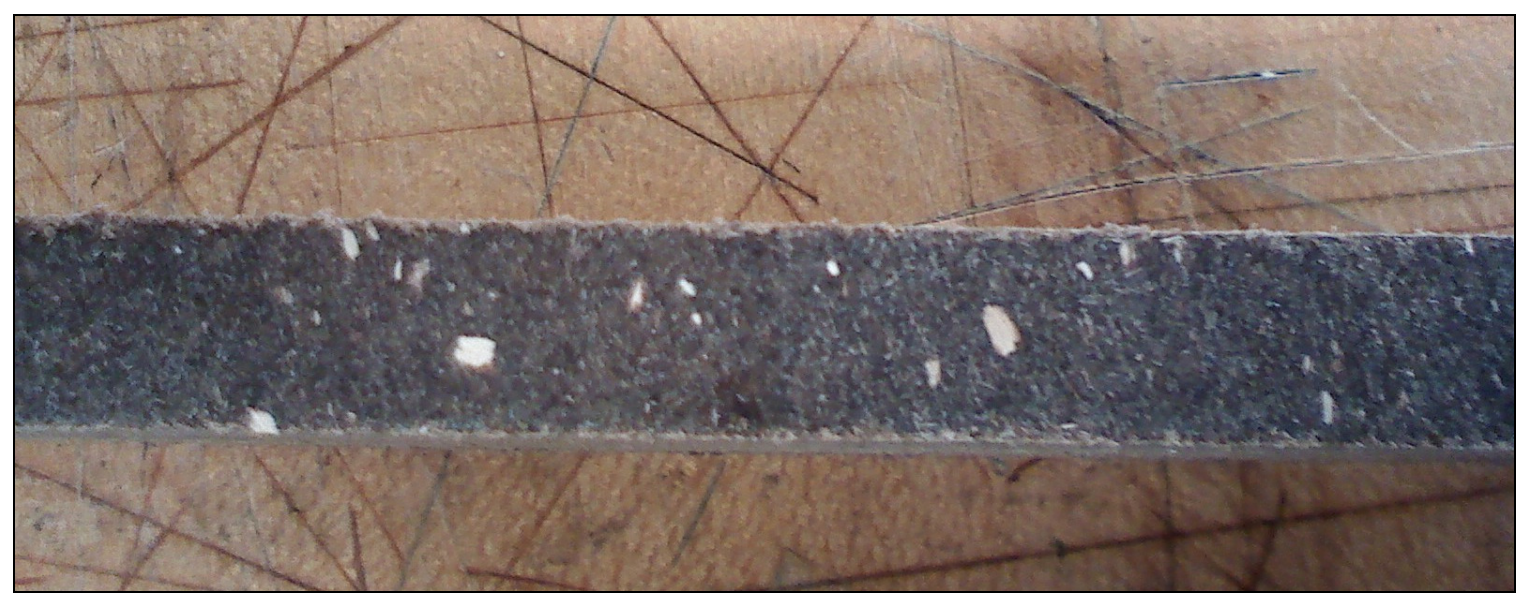

Figure M: Fiber Dispersion

Although some specimens failed prematurely due to fiber pockets, the stress-strain data was still usable when calculating Young's modulus. However, the same data could not be used to calculate ultimate stress. 
The force data was divided by the specimen area to change the forces to nominal stresses. The end result of each tension test was a stress-strain curve which was then fitted with a bilinear curve. A sample stress-strain curve and line fitting is shown in Figure $\mathrm{N}$ below.

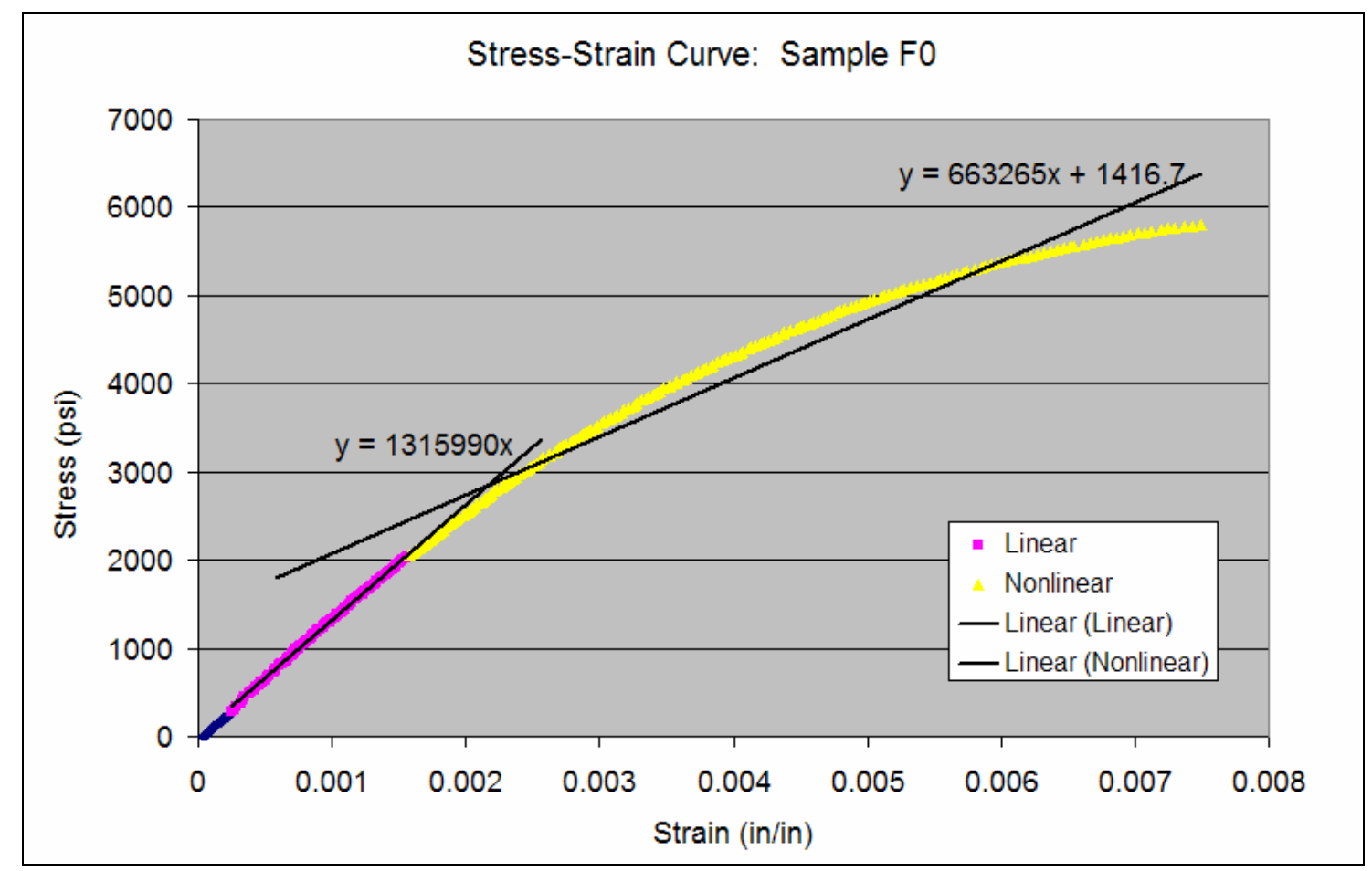

\section{Figure N: Sample Tension Test Stress-Strain Curve}

The slopes of the best fit lines are the values of Young's modulus in both the linearelastic and nonlinear range. All of the modulus values were collected and averaged for each angle. Table 2 on the next page shows the results for the experimental linear modulus $E_{\text {linear }}$, the nonlinear modulus $E_{\text {nonlin }}$, and the bilinear point stress $\sigma_{b}$ as a function of orientation to the strong axis. Graphical summaries of $E_{\text {linear }}$ and $E_{\text {nonlin }}$ are shown in Figures $\mathrm{O}$ and $\mathrm{P}$. 


\begin{tabular}{|c|c|c|c|}
\hline $\begin{array}{c}\text { Angle } \\
\text { (degrees) }\end{array}$ & $\begin{array}{c}\text { Average E } \\
\text { (psinear }\end{array}$ & $\begin{array}{c}\text { Average } \mathbf{E}_{\text {nonlin }} \\
(\mathbf{p s i})\end{array}$ & $\begin{array}{c}\text { Average } \boldsymbol{\sigma}_{\mathbf{b}} \\
(\mathbf{p s i})\end{array}$ \\
\hline \hline 0 & 1064000 & 588000 & 4650 \\
\hline 33 & 735000 & 511000 & 3180 \\
\hline 45 & 634000 & 415000 & 2690 \\
\hline 68 & 517000 & 389000 & 2310 \\
\hline 90 & 495000 & 360000 & 2220 \\
\hline
\end{tabular}

Table 2: Tension Test Summary

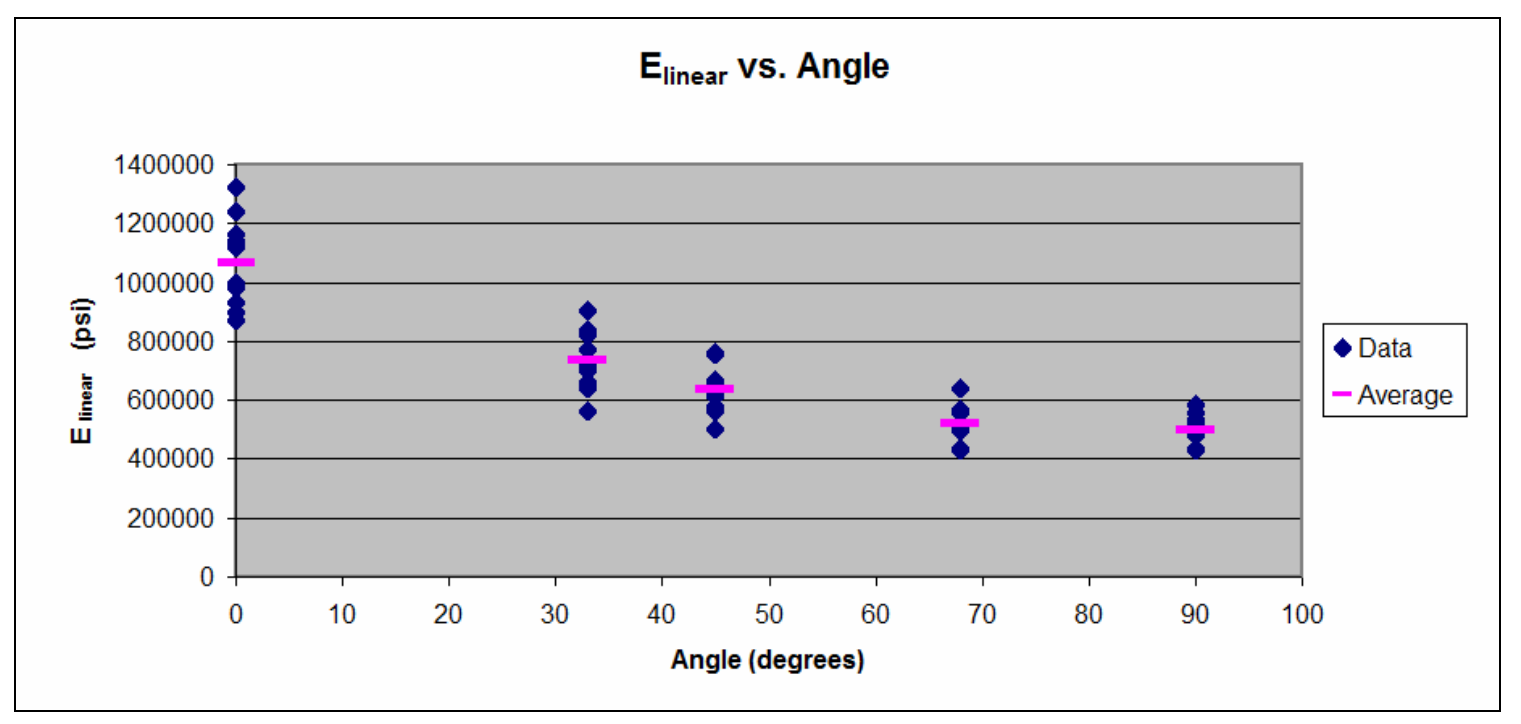

Figure O: Initial Linear Young's Modulus Summary 


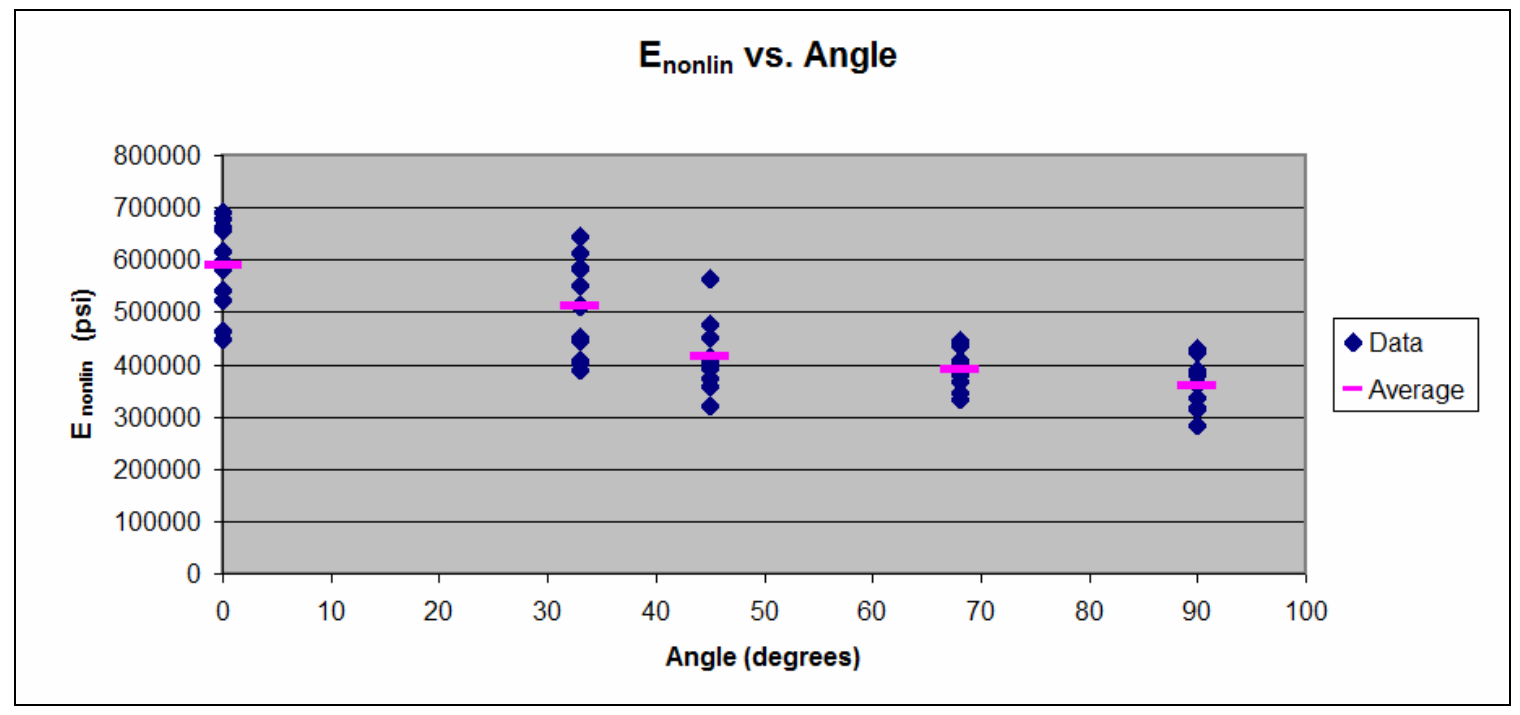

Figure P: Nonlinear Young's Modulus Summary

The most important values to note are the linear-elastic Young's moduli in the strong $0^{\circ}$ and weak $90^{\circ}$ directions, which are 1,064,000 psi and 495,000 psi respectively.

Fifty-six total specimens were tested: 12 at $0^{\circ}, 13$ at $33^{\circ}, 11$ at $45^{\circ}, 10$ at $68^{\circ}$, and 10 at $90^{\circ}$. For each angle, the sample size was increased until the standard deviation was less than $20 \%$ of the value of Young's modulus. As expected, the samples cut closer to $0^{\circ}$ had higher values for Young's modulus and the samples cut closer to $90^{\circ}$ had lower values for Young's modulus. The PP/kenaf planks have a clear strong axis running the direction of the long axis of the planks. Significantly, the values of Young's modulus found for PP homopolymer are almost at the same level as those found for structural timber.

\subsection{Four-Point Bending Test}

Four-point bending tests were performed to determine the linear-elastic shear modulus $G$ of the material. The advantage of this test is that the shear modulus $G$ can be 
found even when Young's modulus $E_{x}$ is unknown. The test setup is shown in Figure Q below.

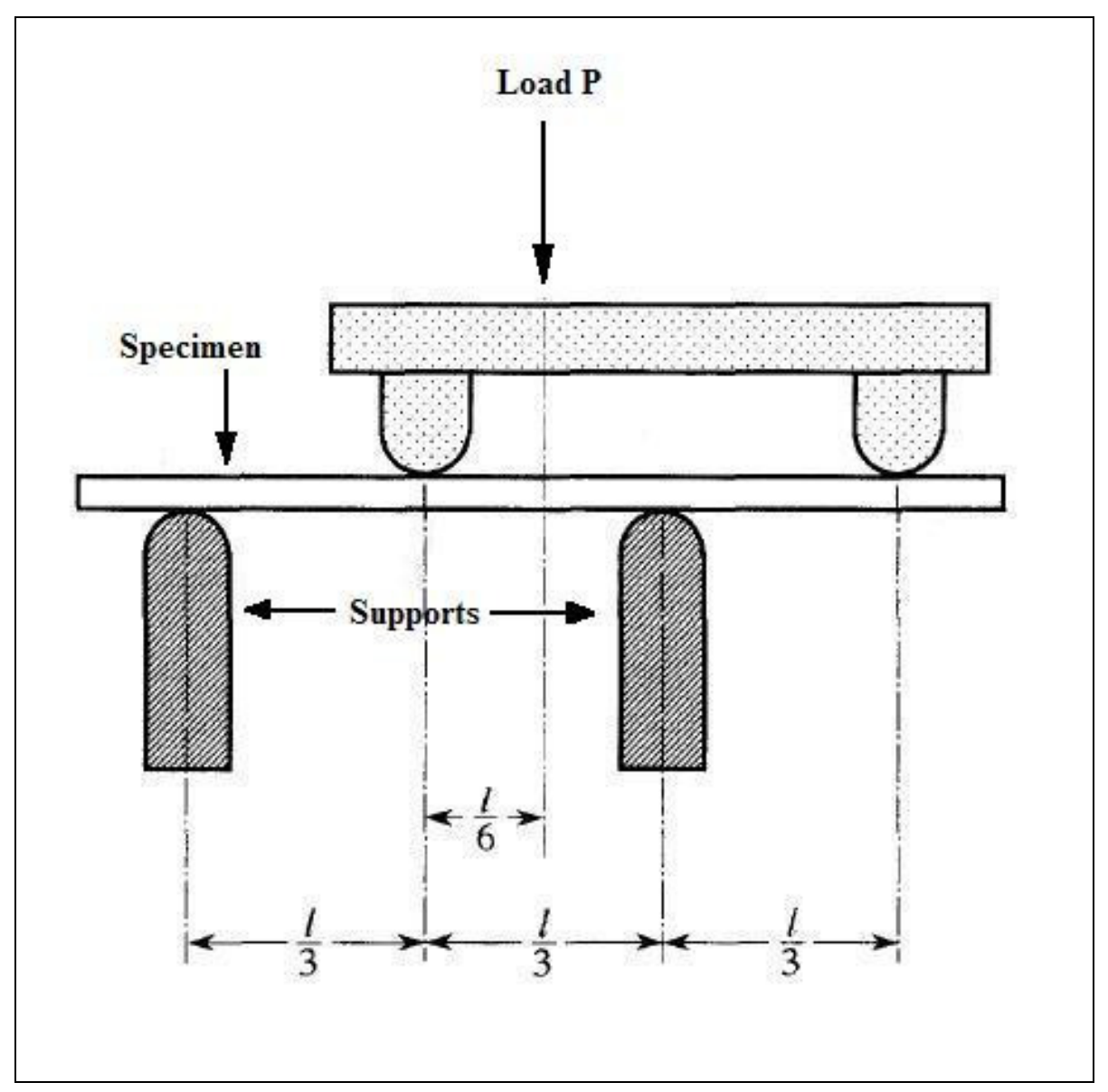

Figure Q: Four-Point Bending Test Setup

Source: Yoshihara 2002

The deflection for the four-point bending test is derived from elastic bending theory and can be found by means of virtual work. The real and virtual shear and moment diagrams are shown in Figure $\mathrm{R}$ on the following page. 


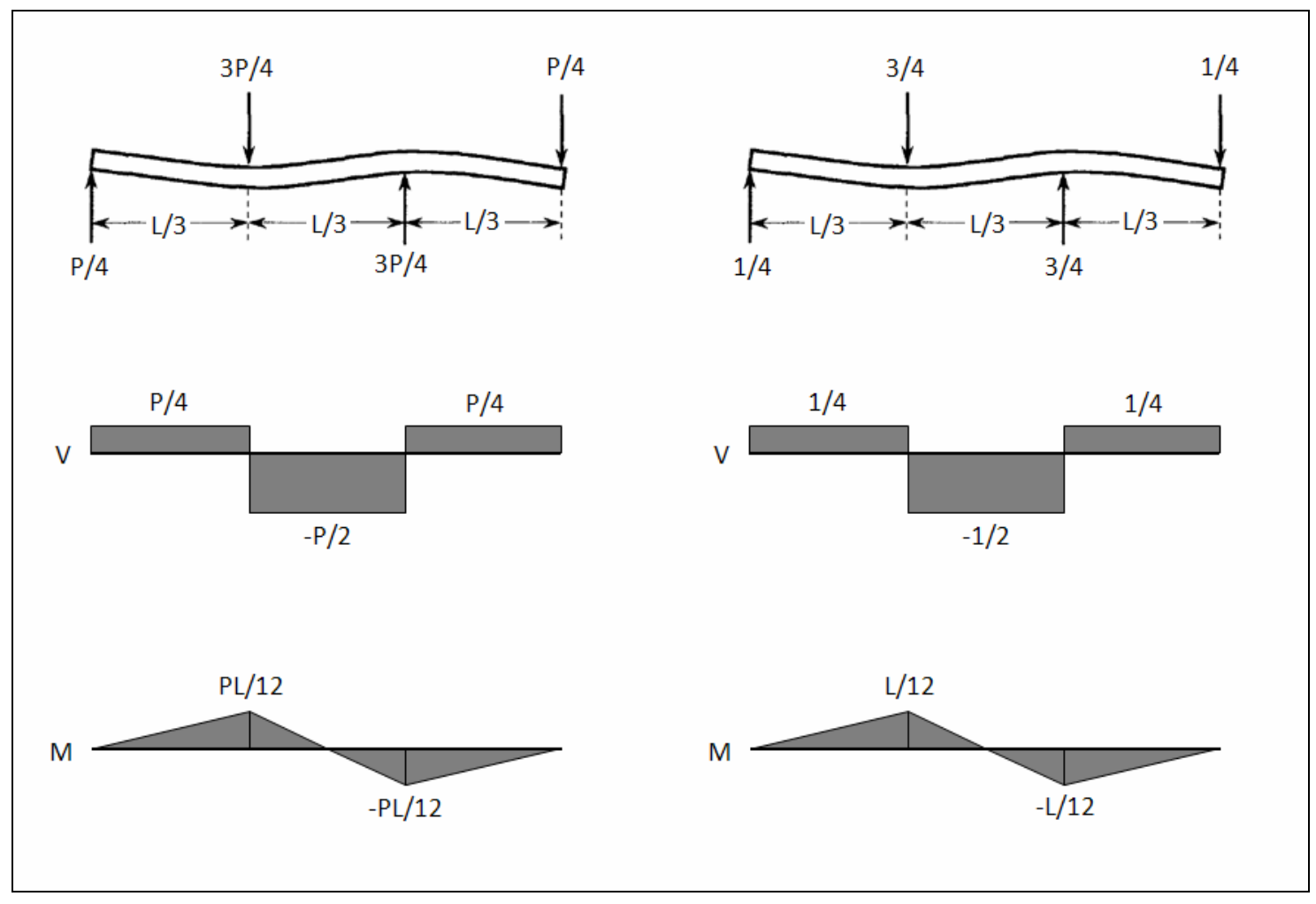

Figure R: Shear and Moment Diagrams for 4-Point Bending Test

The deflection due to bending $\delta_{b}$ and the deflection due to shear $\delta_{s}$ at the points of loading are calculated through integration of the real and virtual shear and moment diagrams as follows:

$$
\begin{gathered}
\delta_{b}=\frac{1}{E I} \int m(x) M(x) d x=\frac{1}{E I}\left[2 \frac{1}{3}\left(\frac{P l}{12}\right)\left(\frac{l}{12}\right)\left(\frac{l}{3}\right)+2 \frac{1}{3}\left(\frac{P l}{12}\right)\left(\frac{l}{12}\right)\left(\frac{l}{6}\right)\right]=\frac{P l^{3}}{432 E I}, \\
\delta_{s}=\frac{s}{G A} \int v(x) V(x) d x=\frac{s}{G A}\left[\left(\frac{P}{4}\right)\left(\frac{1}{4}\right)\left(\frac{l}{3}\right)+\left(-\frac{P}{2}\right)\left(-\frac{1}{2}\right)\left(\frac{l}{3}\right)+\left(\frac{P}{4}\right)\left(\frac{1}{4}\right)\left(\frac{l}{3}\right)\right]=\frac{s P l}{8 G A},
\end{gathered}
$$

Eq. 2

and the total vertical deflection $\delta$ at the points of loading, as shown in Eq. 3, is (Yoshihara 2002) 


$$
\delta=\delta_{b}+\delta_{s}=\frac{P l^{3}}{432 E_{x} I}+\frac{s P l}{8 G A}
$$

Where $P$ is the total load applied,

$l$ is the overall length of the specimen,

$I$ is the moment of inertia of the cross section,

$A$ is the cross-sectional area,

$s$ is Timoshenko's shear factor (1.5 for this experiment),

$E_{x}$ is Young's modulus in the longitudinal direction, and

$G$ is the shear modulus.

If the shear deformation is ignored, the displacement is defined in terms of the apparent Young's modulus $E_{s}$ in Eq. 4 as follows (Yoshihara 2002):

$$
\delta=\frac{P l^{3}}{432 E_{s} I} .
$$

The following relation (Eq. 5) is then obtained from Eq.'s 3 and 4 (Yoshihara 2002):

$$
\frac{1}{E_{s}}=\frac{1}{E_{x}}+4.5 \frac{s}{G}\left(\frac{h}{l}\right)^{2} .
$$

The shear modulus $G$ can be found even if Young's modulus $E_{x}$ is unknown. If $E_{x}$ is known, then $G$ can be solved using simple algebra. However, if $E_{x}$ is not known then $E_{x}$ and $G$ can be solved for simultaneously using varying height-to-length ratios and the method of least squares. This experiment solved for $E_{x}$ and $G$ simultaneously and then compared $E_{x}$ to the $E_{\text {linear }}$ from the tension tests to assess the accuracy of the results.

Two series of tests were performed using the four-point bending setup described by Yoshihara (2002). The first series of tests consisted of four unplaned boards and four planed boards tested at their full length. For this series, only two different $h / l$ (depth/span) ratios were explored and more testing was needed. Series two of testing consisted of eight more specimens with varying $h / l$ ratios. 
The boards were tested using the universal testing machine (UTM). The UTM is controlled manually and displays force and vertical displacement values. The test setup is shown in Figure $\mathrm{S}$ below.

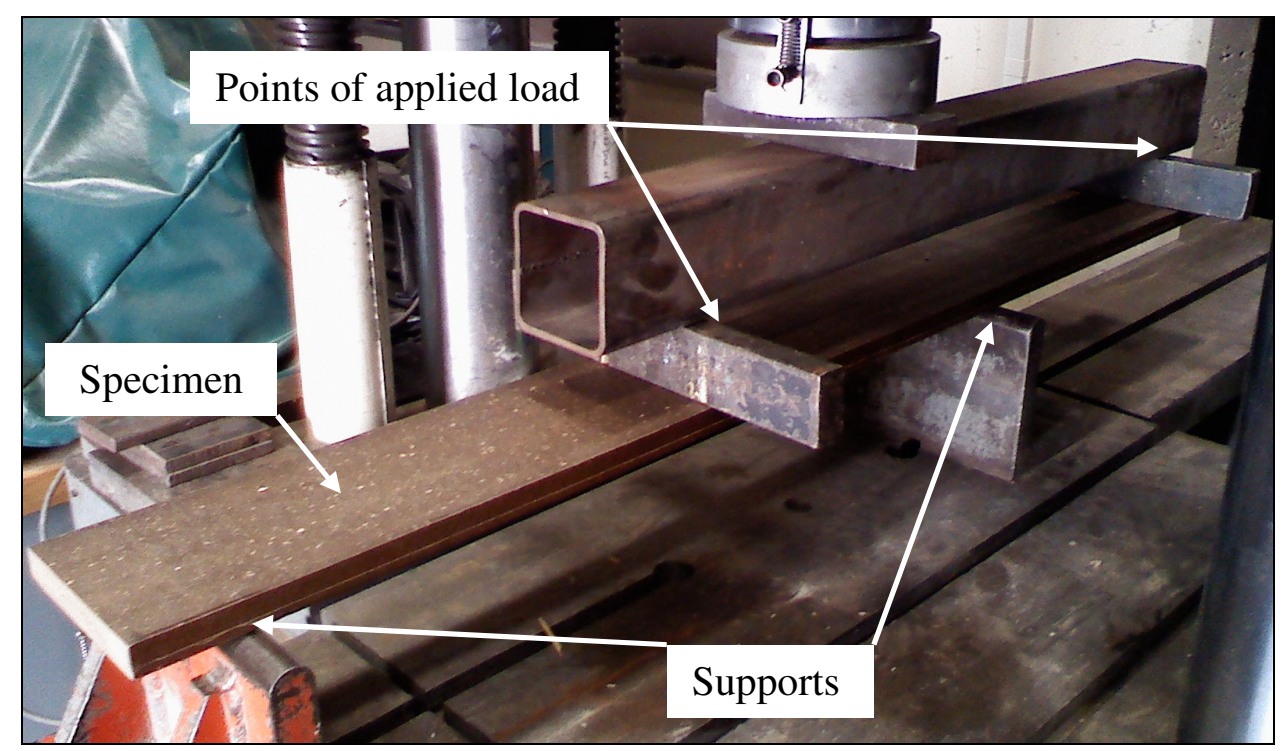

Figure S: Four-Point Bending Test Setup

In this experiment, the specimens were tested in shear in the 1-3 plane; see Figure $\mathrm{F}$ on page 14. This orientation was chosen because it required the least specimen preparation, since the span was suggested to be 20 times larger than the depth (Yoshihara 2002). Therefore this experiment would yield a shear modulus for the 1 axis in the 3 direction $G_{13}$

The supports and loads were alternated and placed at third-points of the beam. Total beam lengths that were used were 18", 25.5" and 42". The internal loading point takes $3 / 4$ of the total load and the cantilevered loading point takes $1 / 4$ of the total load. In theory, the two loading points displace the exact same amount. The two load point 
displacements were checked to be the same using dial gauges as shown in Figure T, below.

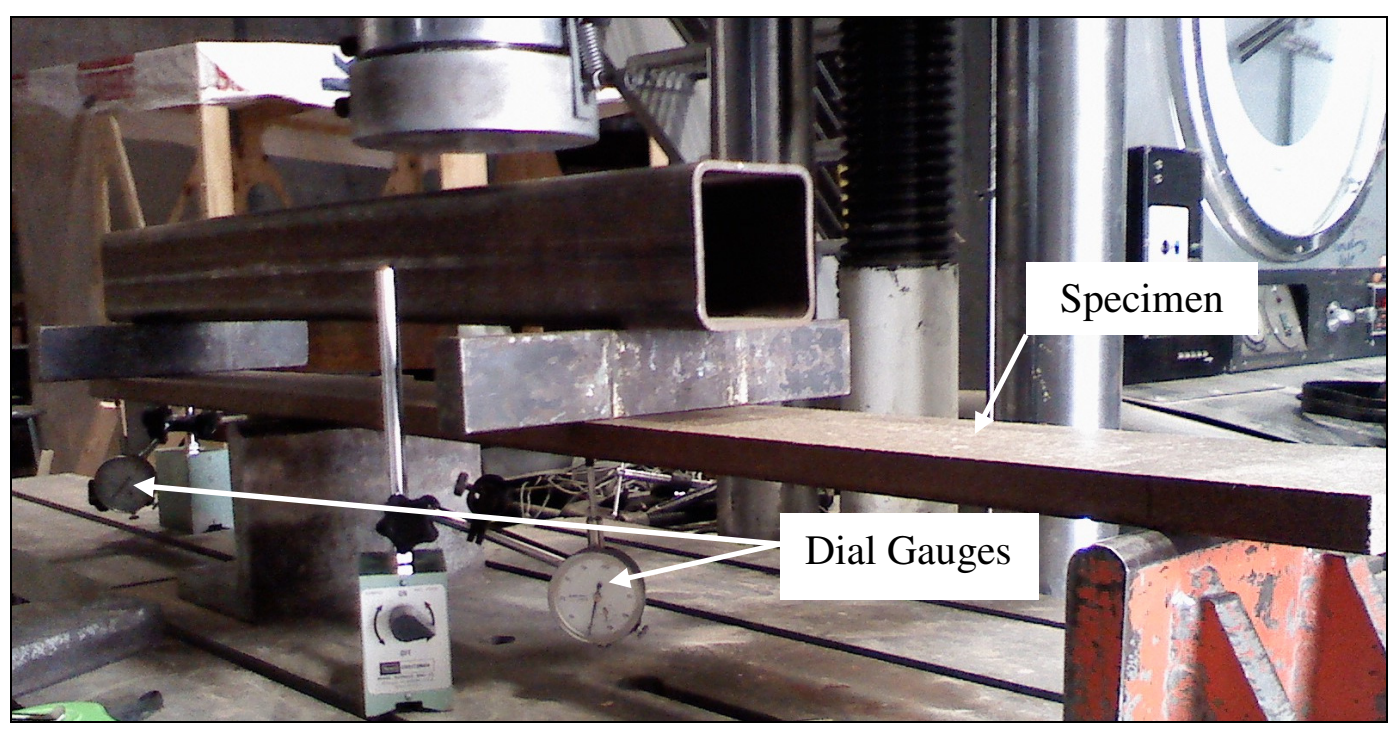

Figure T: Displacement Check

For this check, both gauges were visually read and recorded at a given load. The

difference between the two displacements was found to be less than 5\%. Therefore it was assumed that both points displace the same amount. Also, the displacement given digitally by the universal testing machine was shown to match the displacement given by the dial gauges.

The load $P$ was the applied load from the UTM. Both the load and the vertical head displacement were recorded manually by reading the load gauge and head deflection display. The UTM output devices are shown in Figure U on page 28, following. 


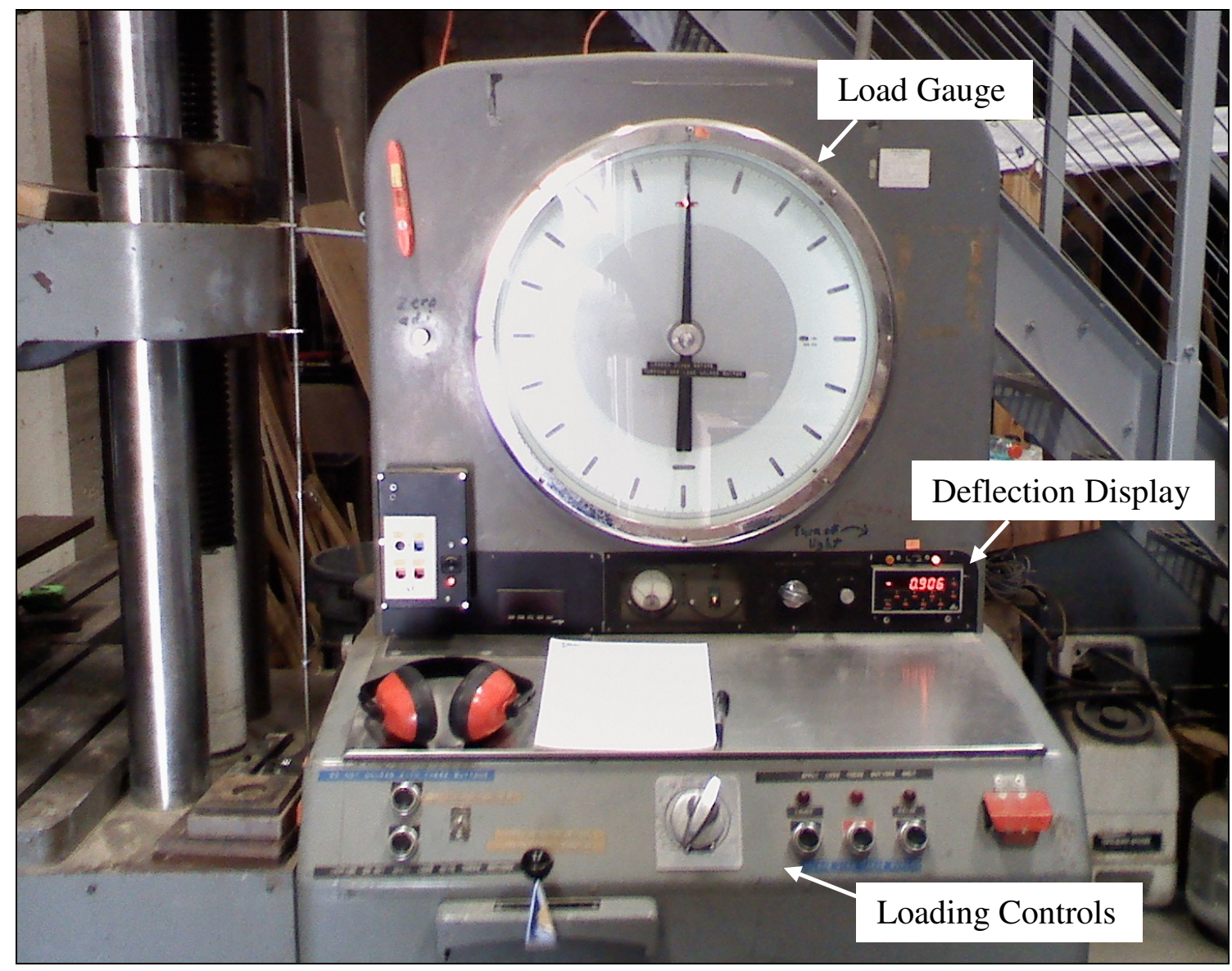

Figure U: Universal Testing Machine Readouts

For each test, deflection vs. load data was collected at increments of 25 and 50 lbs. Since the four-point bending method works best when the material is in the linear-elastic range, data was collected at smaller load increments in the initial stages of loading.

In the first series of tests, four planks were tested without modification, and four of the planks were planed to make the section rectangular. The width of each board was 5.125". The moment of inertia of an unplaned board was found by hand to be 0.479 in $^{4}$. Subsequently, the thickness of the planed boards was reduced to $0.828 "$, and the moment of inertia to $0.242 \mathrm{in}^{4}$. Figure $\mathrm{V}$ on the following page shows an unmodified crosssection. 


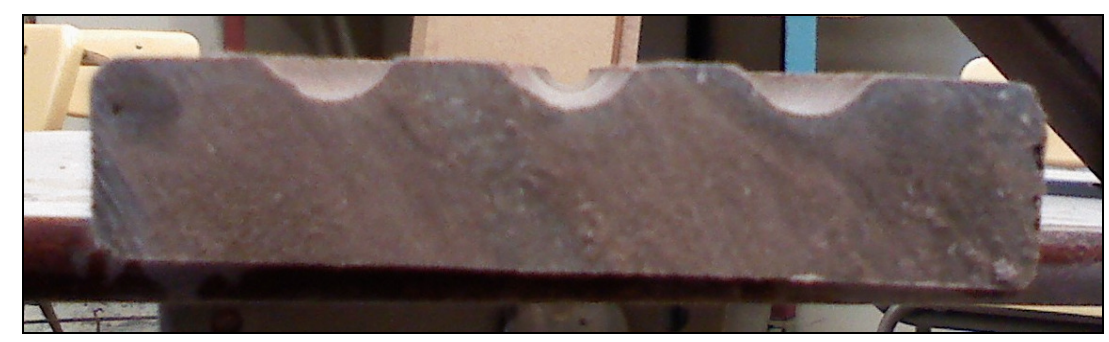

Figure V: Cross-Section of Unplaned Board

As seen in the figure above, the exact height, or thickness, of the unplaned board was difficult to measure because of the uneven top surface. Therefore, exact $h / l$ ratios could not be determined and the unplaned board tests were ultimately not used in determining $G$. Specimen 23 was the only specimen taken from the first series of testing because that series of testing only included one useful $h / l$ ratio. In the second series of testing, the widths of the boards were 4.453 " or 4.328 ", and the heights of the boards varied among 0.664", 0.781", 0.836", and 0.844". The final range of $h / l$ ratios used was 0.0197 to 0.0469 .

$E_{s}$, the apparent Young's modulus assuming only bending deformation, needed to be calculated for each test specimen using the load-deflection data. Eq. 4 on page 25 was rearranged to the slope-intercept form of a line as follows:

$$
\frac{P l^{3}}{432 I}=E_{s} \delta \text {. }
$$

In this form, the left hand side represents the dependent variable, $\delta$ represents the independent variable, and $E_{s}$ is the slope of the line. For each test, the left hand side was plotted as a function of $\delta$, and the slope was recorded as $E_{s}$. A sample plot is shown in Figure $\mathrm{W}$ on the following page. 


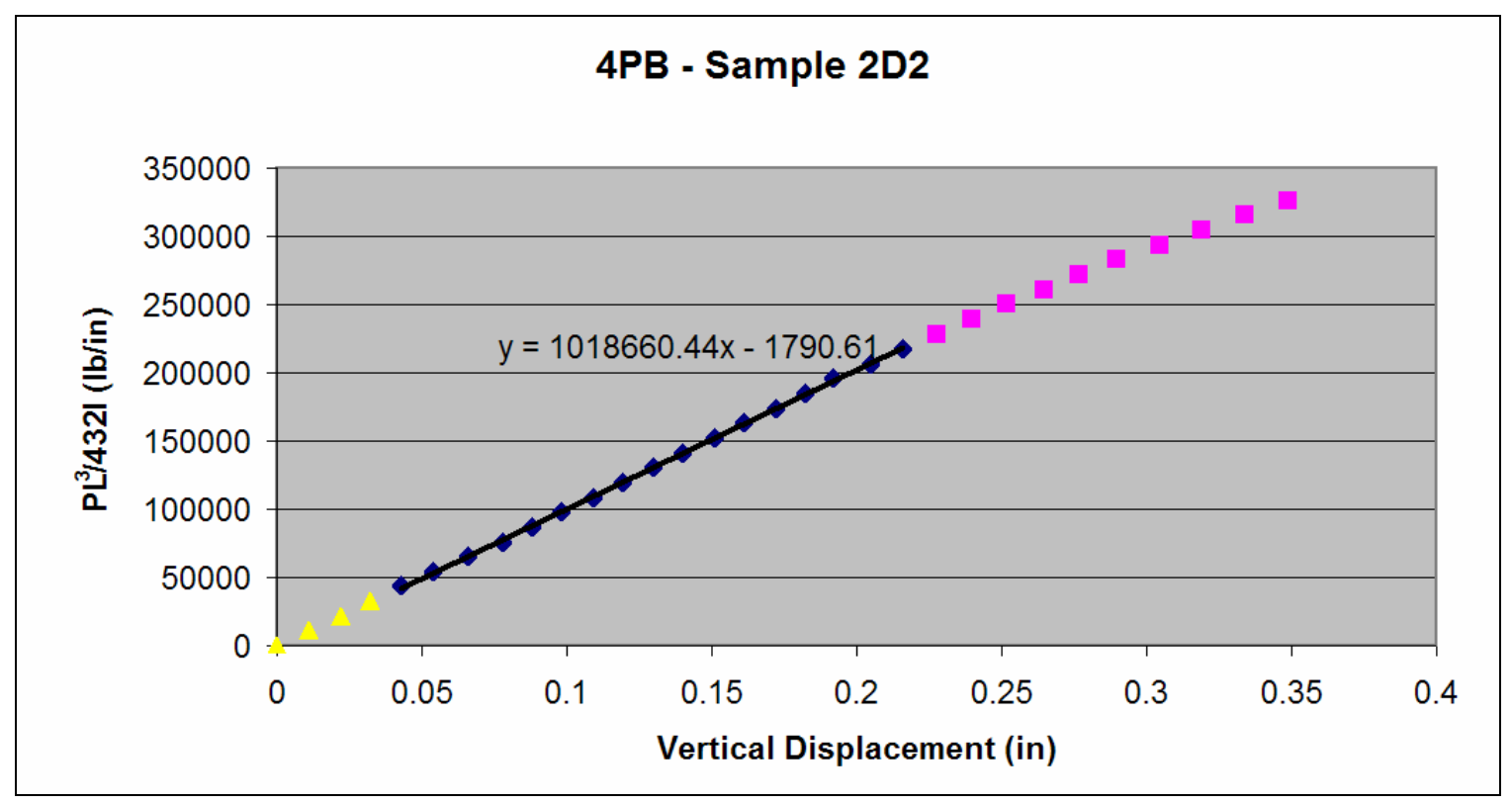

Figure W: Sample Plot of $\delta$ vs. $P l^{3} / 432 I$, Eq. 6, for a Specimen

A best fit line was used to determine $E_{s}$, the slope, for each plot. Table 3 below gives a summary of the specimens, their respective $h / l$ ratios and $E_{s}$ values.

\begin{tabular}{|c|c|c|c|c|c|}
\hline Specimen Label & width (in) & height (in) & length (in) & h/l (in/in) & $\mathbf{E}_{\mathbf{s}}(\mathbf{p s i})$ \\
\hline \hline 23 & 5.125 & 0.828 & 42.0 & 0.0197 & 1006054 \\
\hline 2A1 & 4.453 & 0.844 & 18.0 & 0.0469 & 906425 \\
\hline 2B1 & 4.453 & 0.664 & 18.0 & 0.0369 & 1006502 \\
\hline 2C1 & 4.453 & 0.836 & 18.0 & 0.0464 & 929482 \\
\hline 2D1 & 4.328 & 0.781 & 18.0 & 0.0434 & 943069 \\
\hline 2A2 & 4.453 & 0.844 & 25.5 & 0.0331 & 975654 \\
\hline 2B2 & 4.453 & 0.664 & 25.5 & 0.0260 & 1050863 \\
\hline 2C2 & 4.453 & 0.836 & 25.5 & 0.0328 & 1081665 \\
\hline 2D2 & 4.453 & 0.781 & 25.5 & 0.0306 & 1018660 \\
\hline
\end{tabular}

Table 3: 4pt Bending Experiment Specimen Summary

Then, Eq. 5 from page 25 was used, with the varying $E_{s}$ and $h / l$ ratios, to solve simultaneously for $E_{x}$ and $G$. Rearranging Eq. 5 gives 


$$
G=4.5 \frac{s}{\frac{1}{E_{s}}-\frac{1}{E_{x}}}\left(\frac{h}{l}\right)^{2}
$$

Substituting the values of $h / l$ and the corresponding $E_{s}$ for all nine specimens gave a set of nine equations with two unknowns, $G$ and $E_{x}$. All nine equations were then simultaneously plotted on one graph of $G$ as a function of $E_{x}$, as shown in Figure X below.

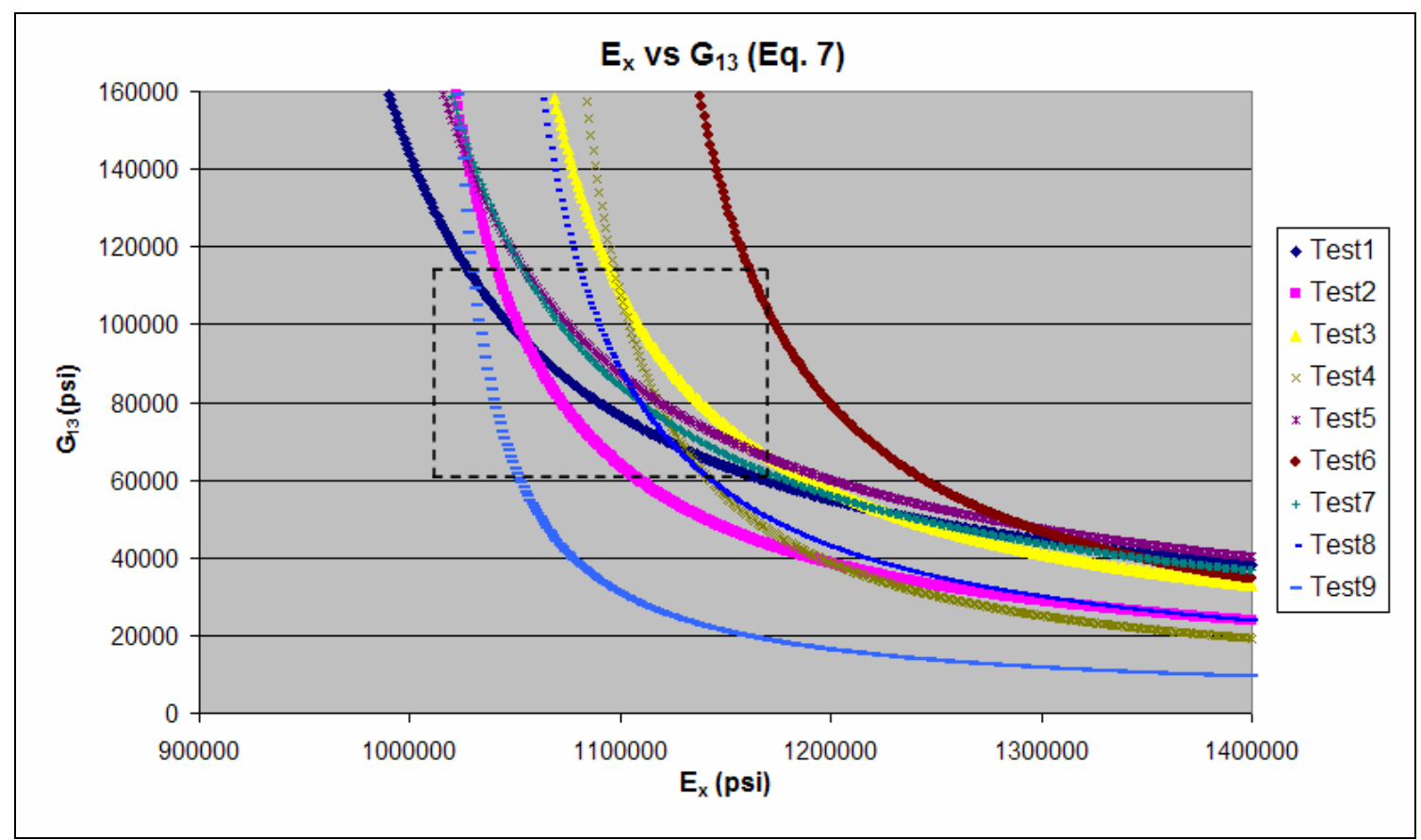

Figure X: Plot Used in Solving for $E_{x}$ and $G_{13}$

Theoretically, all nine functions would cross at the same point and the values of $E_{x}$ and $G$ could be read straight off the graph. However, as experimental results aren't perfect, statistics were needed to determine the desired values. The highlighted area in the graph above shows where many of the functions cross and where the values of $E_{x}$ and $G$ will fall. The method of least squares was used to find values of $E_{x}$ and $G$ that most closely 
satisfied all nine functions at the same time and these values were found to be $E_{x}=$ $1,087,000$ psi and $G_{13}=97,000$ psi.

In conclusion, the values found from this test were reliable. There is less than a $3 \%$ difference between Young's modulus found from this test $\left(E_{x}=1,087,000\right)$ and Young's modulus found from the tension tests $\left(E_{\text {linear }}=1,064,000\right)$. This finding helps to validate this experiment and verify the value found for the shear modulus $G_{13}$.

\subsection{Simulated Four-Point Bending Test}

Four-point bending tests were simulated using a finite element analysis (FEA) in order to assess the accuracy of the four-point bending test and verify the results of the physical experiment.

The experimental values found for Young's modulus and the shear modulus, from the four-point bending experiment, were input as material properties in the finite element model to show that the simulated experiment predicts these same values. The beam was modeled in the finite element computer program ANSYS, using beam elements. The material properties used in these simulations were defined as $E_{x}$ for Young's modulus in the longitudinal direction, $G_{x y}$ for the shear modulus, $v_{x y}$ for Poisson's ratio, and $s$ for the shear factor; the values of these constants were as follows:

$$
\begin{aligned}
& E_{x}=1,064,000 \mathrm{psi}, \\
& G_{x y}=97,000 \mathrm{psi}, \\
& v_{x y} \text { is estimated as } 0.1, \text { and } \\
& s=1.2 .
\end{aligned}
$$

Because there was no experimental data for Poisson's ratio for this material, the value was estimated based on known values for wood materials. The deflection of the four- 
point bending test does not depend on Poisson's ratio and therefore has no effect in this experiment; however, ANSYS required that it be defined to run the analysis.

The initial set of tests was run with the more commonly known shear factor of 1.2 instead of 1.5 as suggested by Yoshihara. The test setup and meshed beam elements are shown in Figure Y below.

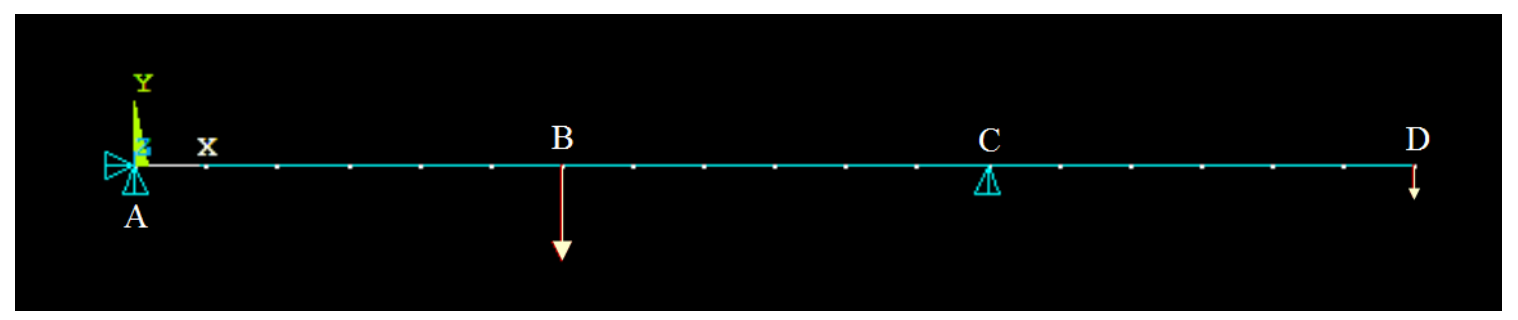

Figure Y: Simulated Experiment Test Setup and Beam Mesh

Three fourths of the total load is applied to the inner loading point B while one fourth of the total load is applied to the outer loading point D. Deflection can be measured at either loading point because they both displace the same amount. For each specimen, a total load of 20 pounds was applied and the resulting deflection was recorded. Then, $\mathrm{E}_{\mathrm{s}}$ for each specimen was solved for using Eq. 6. A summary of the specimens used and their corresponding $h / l$ ratios and $E_{s}$ values is shown in Table 4 on the following page. 


\begin{tabular}{|c|c|c|c|c|c|}
\hline Specimen Label & width (in) & height (in) & length (in) & h/l (in/in) & E $_{\mathbf{s}}$ (psi) \\
\hline \hline 1 & 4.000 & 0.800 & 16 & 0.0500 & 897795 \\
\hline 2 & 4.453 & 0.844 & 18 & 0.0469 & 915032 \\
\hline 3 & 4.453 & 0.664 & 18 & 0.0369 & 966612 \\
\hline 4 & 4.453 & 0.836 & 18 & 0.0464 & 917482 \\
\hline 5 & 4.453 & 0.781 & 18 & 0.0434 & 933818 \\
\hline 6 & 4.453 & 0.844 & 24 & 0.0342 & 974745 \\
\hline 7 & 4.453 & 0.664 & 24 & 0.0277 & 1006923 \\
\hline 8 & 4.453 & 0.836 & 24 & 0.0348 & 976292 \\
\hline 9 & 4.453 & 0.781 & 24 & 0.0325 & 986644 \\
\hline 10 & 4.000 & 0.800 & 36 & 0.0222 & 1026460 \\
\hline 11 & 4.000 & 1.000 & 36 & 0.0278 & 1006492 \\
\hline 12 & 4.000 & 1.200 & 36 & 0.0333 & 983117 \\
\hline 13 & 4.000 & 1.800 & 36 & 0.0500 & 897795 \\
\hline
\end{tabular}

\section{Table 4: 4pt Bending Simulated Experiment Specimen Summary}

Then Eq. 7 was used to plot $G$ as a function of $E_{x}$ for each specimen, shown in Figure Z below.

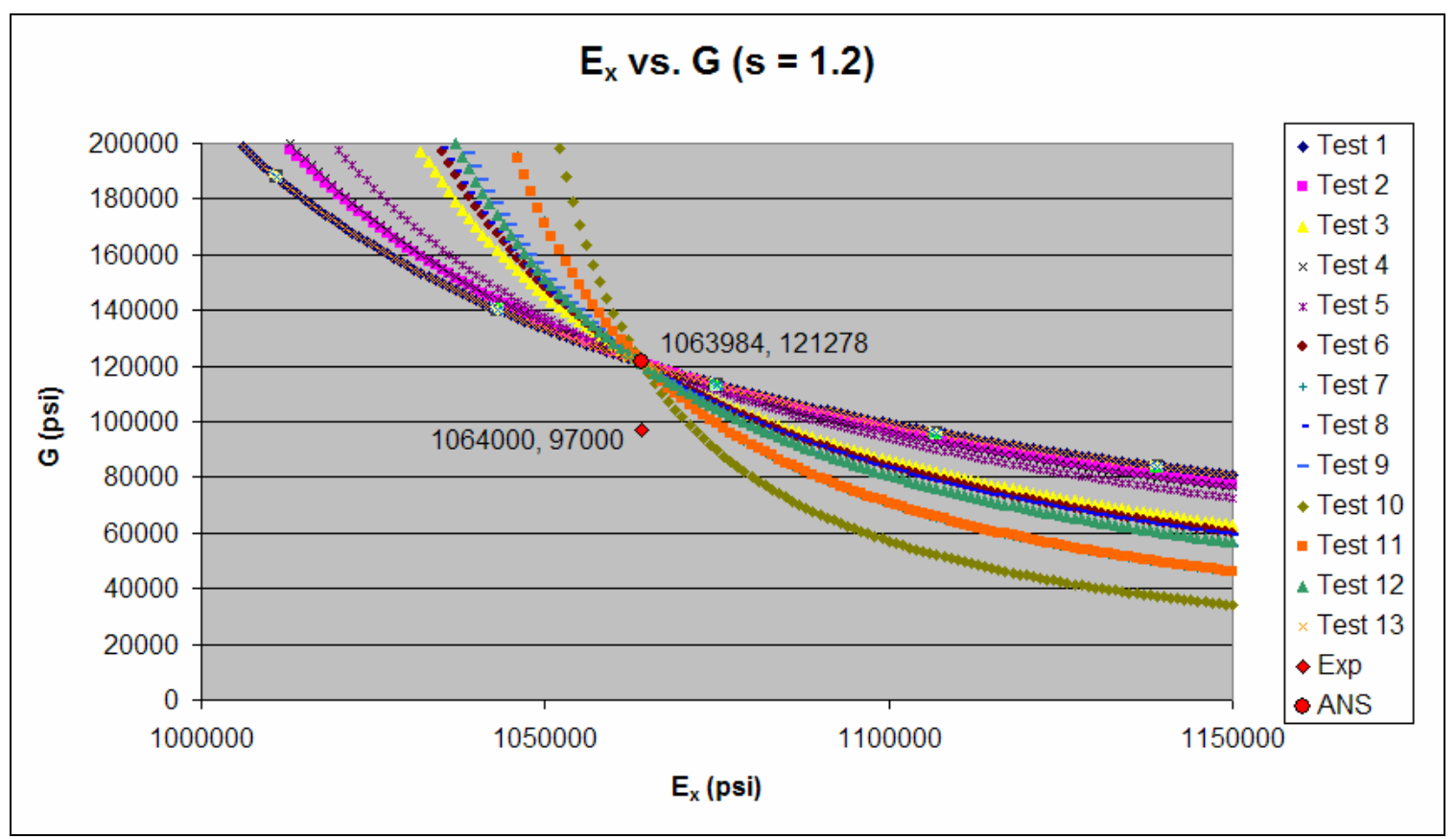

Figure Z: Plot Used in Solving for $E_{x}$ and $G$ for Simulated 4pb Experiment $(s=1.2)$

Nonlinear Modeling of a Sustainable Material 
The resultant $E_{x}=1,063,984$ psi and $G=121,278$ psi are easily read off the plot.

However, the value for $G$ is off by a factor of 1.25 which is also the ratio of the two shear factors, 1.5 and 1.2. Another set of tests were run with the shear factor equal to 1.5 and the resultant $E_{x}$ vs. $G$ plot is shown in Figure AA.

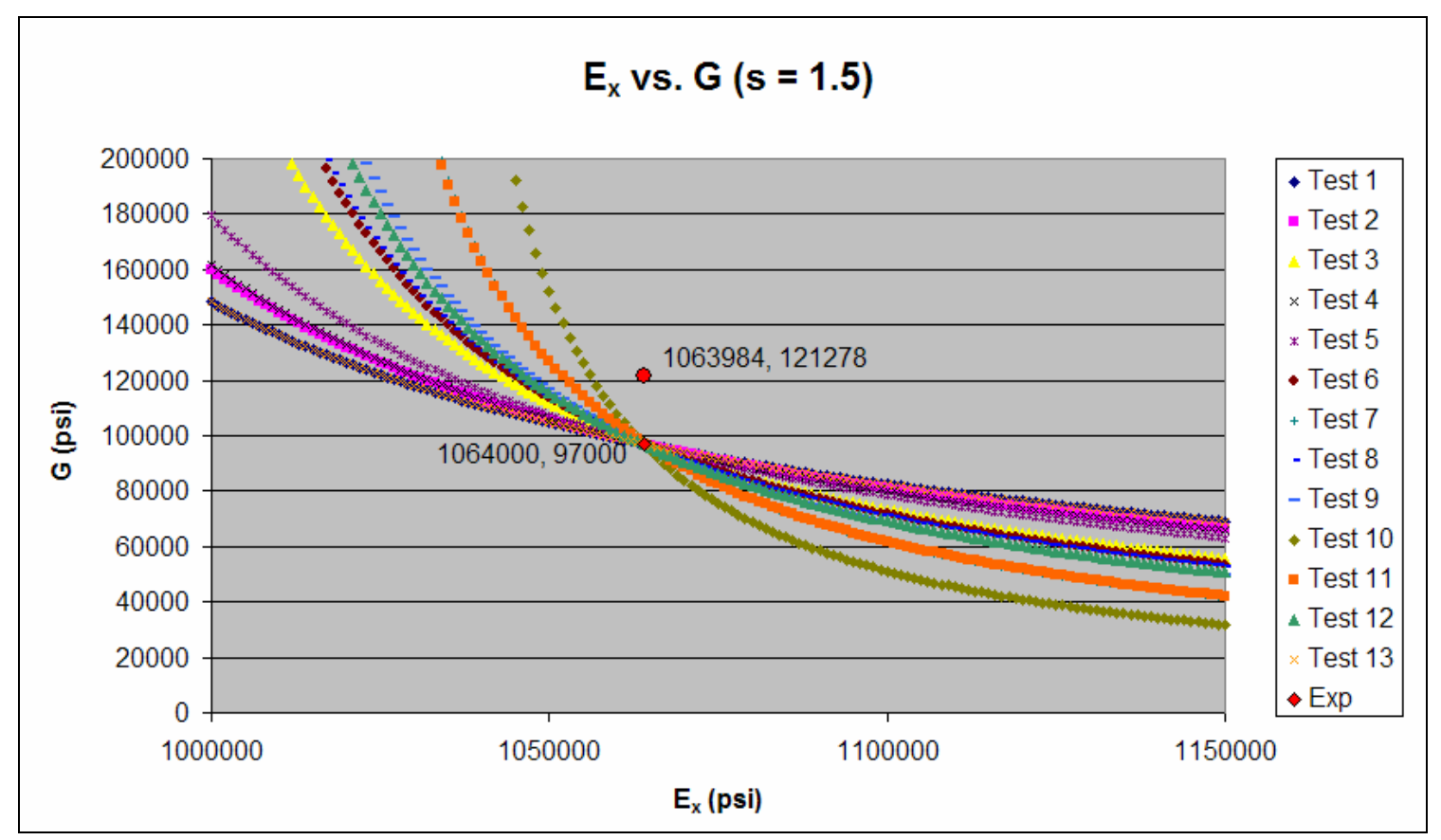

Figure AA: Plot Used in Solving for $E_{x}$ and $G$ for Simulated 4pb Experiment ( $s=$ 1.5)

With a shear factor of 1.5 , the moduli are predicted as $E_{x}=1,064,000 \mathrm{psi}$ and $G=$ 97,000 psi. These results show that, theoretically, the four-point bending test can accurately predict Young's modulus $E_{x}$ and the shear modulus $G$ when using a shear factor of 1.5. Therefore, my experimental values for the longitudinal Young's modulus and shear in the 1-3 plane are reliable. 


\subsection{Experiment Results}

It was initially thought that $G_{12}$ would not differ greatly from $G_{13}$. For wood, the properties in the two weak axes are very similar to each other. However, evidence shows that $G_{12}$ is much larger than $G_{13}$ for this material. Since a four-point bending experiment was not performed in the 1-2 plane, the shear modulus $G_{12}$ was extracted from the classic orthotropic elasticity equation, Eq. 8 (Jones 1975).

$$
\frac{1}{E_{\theta}}=\frac{\cos ^{4} \theta}{E_{1}}+\frac{\sin ^{4} \theta}{E_{2}}-\frac{2 \nu_{12} \cos ^{2} \theta \sin ^{2} \theta}{E_{1}}+\frac{\cos ^{2} \theta \sin ^{2} \theta}{G_{12}},
$$

where $E_{1}$ is known,

$E_{2}$ is known, $v_{12}$ is estimated as 0.1 , $E_{\theta}$ is known for certain values of $\theta$, and $G_{12}$ is unknown.

In this equation, the value of Poisson's ratio was based on known wood values and is inversely related to the shear modulus. Therefore, if $v_{12}$ was estimated to be larger than 0.1 , the resulting shear modulus $G_{12}$ would be smaller.

$E_{\theta}$ was plotted as a function of $\theta$, and a value of $G_{12}$ was found that would minimize the error between Eq. 8 and the tension experiment data. A plot of this equation, with two different values of $G_{12}$, along with the tension data is shown in Figure BB. 


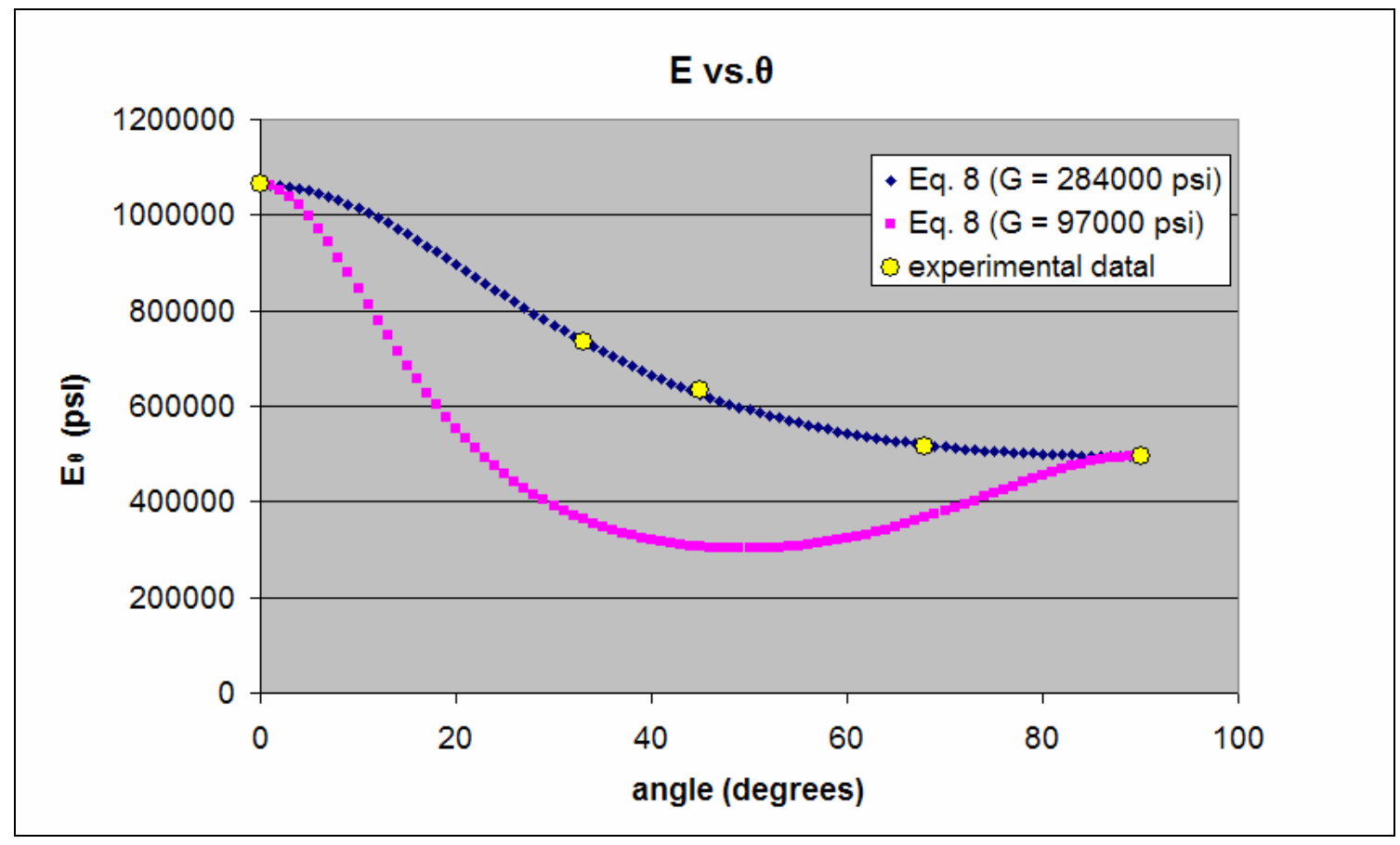

Figure BB: Fitting Eq. 8 to Experimental Data

$G_{12}$ is found to equal 284,000 psi when using Eq. 8. Assuming that $G_{12}=G_{13}$ would show that the classic orthotropic elasticity equation predicts a shear modulus with an error of $193 \%$, too large to be entirely assigned to the inapplicability of the equation to our material. Therefore, the plastic-fiber composite material must have different properties in the 2 and 3 axes. Further testing in both axes is needed to support this claim. For the purpose of this thesis, it is assumed that $G_{12}=284,000$ psi and $G_{13}=97,000 \mathrm{psi}$.

Reasons for differing behavior in the 2 and 3 axes may be partly attributed to the length of the fibers. In wood, the length of the cells ensures that all of them lie in the same general direction, giving it the properties of one strong axis and two similarly weaker orthogonal axes. The fibers in this material are much smaller, $\approx 1 \mathrm{~mm}$, and may not all align perfectly in one direction. When this 1.125 " x 5.125 " board is extruded, the 
fibers may align most towards the 1 axis, second most towards the 2 axis, and the least towards the 3 axis. 


\subsection{CONSTITUTIVE MODEL}

The stress-strain constitutive model for this plastic-fiber composite was developed from two known wood-based constitutive models. The proposed constitutive model predicts the bilinear stress-strain relationship at any orientation to the strong axis, given a nonlinear or bilinear stress-strain relationship in the strong axis.

\subsection{First Wood-Based Constitutive Model}

Research was done to find a constitutive model for this material that could predict the linear-elastic Young's modulus at varying orientations to the strong axis. Three models were considered, and one was chosen that best matched the experimental tension test data for the 1-2 plane.

The first model considered was the classic orthotropic elasticity equation, Eq. 8, which was shown to fit the experimental data when the shear modulus was 284,000 psi. This model was ultimately not chosen because of the dependence on the shear modulus $G$, which was not solved for experimentally in the 1-2 plane.

The second model considered for this material is the empirical relationship for plywood-type panels proposed by Saliklis (2000). This relationship, shown in Eq. 9 below, looks similar to the orthotropic elasticity equation except without the Poisson's ratio term.

$$
\frac{1}{E_{\theta}}=\frac{\cos ^{4} \theta}{E_{1}}+\frac{\sin ^{4} \theta}{E_{2}}+\frac{\cos ^{2} \theta \sin ^{2} \theta}{\left(A^{2 A}\right) G_{12}},
$$

where $A=E_{2} / E_{1}$, and

$G_{12}=284,000$ psi.

A plot of Eq. 9 against the experimental data is shown in figure $\mathrm{CC}$ on the next page. 


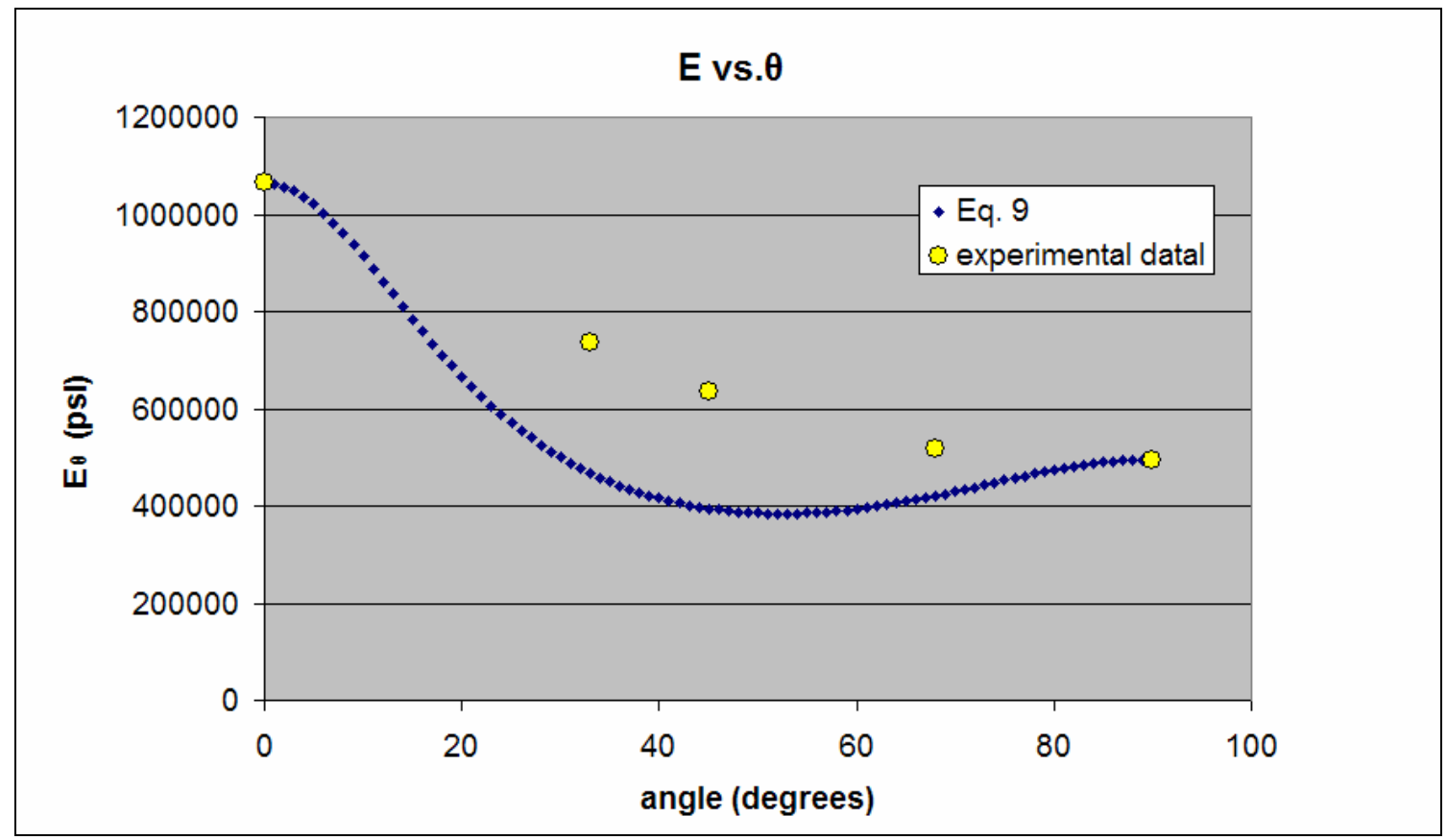

Figure CC: Fitting Eq. 9 to Experimental Data

This model underestimates Young's modulus between the strong and weak axes because it was calibrated to work for plywood, which is strongest at $0^{\circ}$ and $90^{\circ}$, but weaker in between.

The final model tested for this material was the empirically derived Hankinson's formula shown below.

$$
E_{\theta}=\frac{E_{1} E_{2}}{E_{1} \sin ^{n} \theta+E_{2} \sin ^{n} \theta} .
$$

This model has been shown to work well for many different species of wood, with the exponent value $n$ varying between 1.5 and 2. A minimization of error approach was used to find the value $n=1.8$ for this plastic-fiber composite. Figure DD on the next page shows Eq. 10, with $n=1.8$ and $n=2$, against the experimental data. 


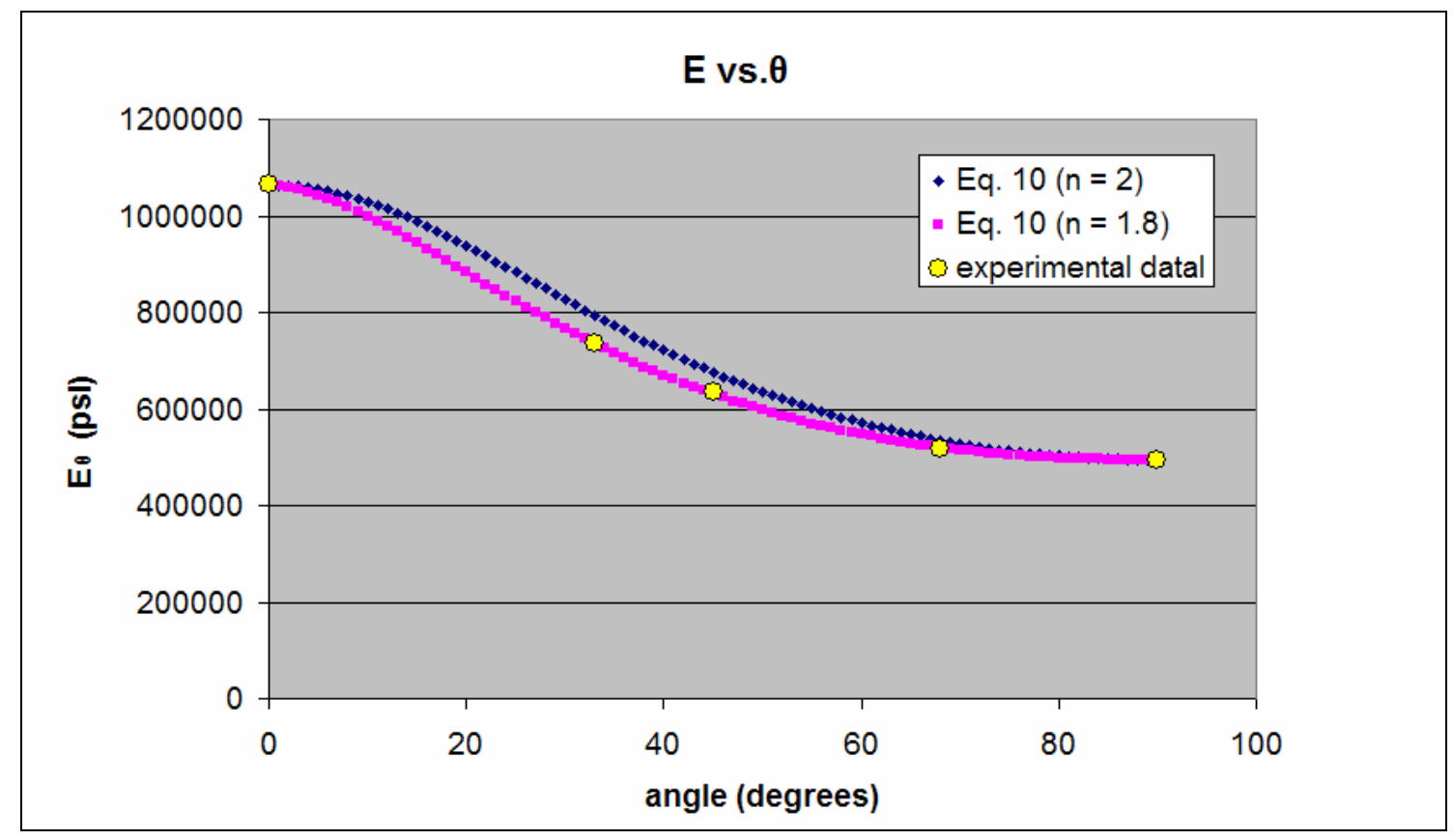

Figure DD: Fitting Eq. 10 to Experimental Data

Hankinson's formula provides the best match to the tension experiment data, whether $\mathrm{n}=$ 2 or 1.8 , and was chosen as the best model that predicts the linear-elastic Young's modulus at varying angles for this material. The only drawback with using Hankinson's formula is that it cannot be used to solve for the shear modulus.

\subsection{Second Wood-Based Constitutive Model}

Further research was done to find a constitutive model that could predict bilinear stress-strain curves in the 1 and 2 axes. The model that was considered was the one proposed by Saliklis (2003). This model can take a bilinear stress-strain curve in the 1 axis and predict the bilinear stress-strain curve for the 2 axis or vice versa. It can also be used to predict the bilinear stress-strain relationship for shear in the 1-2 plane. The bilinear stress-strain relationship is shown in figure EE. 


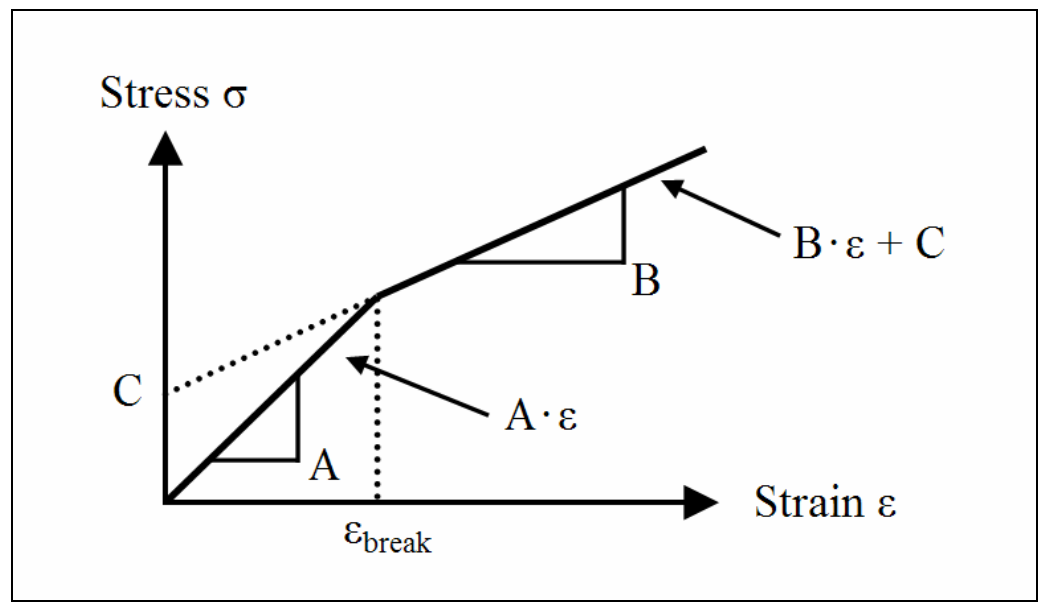

Figure EE: Bilinear Constitutive Model

In this model, A, B and C describe the two straight lines of the stress versus strain curve.

First, the bilinear stress-strain relationship for the 2 axis is defined as follows:

$$
\begin{aligned}
& \sigma_{2}\left(\varepsilon_{2}\right)=A_{2} \varepsilon_{2} \quad \text { for } \quad \varepsilon_{2} \leq \frac{C_{2}}{A_{2}-B_{2}} \\
& \sigma_{2}\left(\varepsilon_{2}\right)=B_{2} \varepsilon_{2}+C_{2} \quad \text { for } \quad \varepsilon_{2} \geq \frac{C_{2}}{A_{2}-B_{2}}
\end{aligned}
$$

Next, the bilinear stress-strain prediction for the 1 axis is as follows:

$$
\begin{array}{ll}
\sigma_{1}\left(\varepsilon_{1}\right)=A_{1} \varepsilon_{1} \quad \text { for } \quad & \varepsilon_{1} \leq \frac{C_{2}}{A_{2}-B_{2}} \sqrt{\frac{v_{2}}{v_{1}}} \\
\sigma_{1}\left(\varepsilon_{1}\right)=B_{1} \varepsilon_{1}+C_{1} \quad \text { for } \quad & \varepsilon_{1} \geq \frac{C_{2}}{A_{2}-B_{2}} \sqrt{\frac{v_{2}}{v_{1}}}
\end{array}
$$

where

$$
\begin{aligned}
& A_{1}=A_{2} \frac{v_{1}}{v_{2}}, \\
& B_{1}=B_{2} \frac{v_{1}}{v_{2}},
\end{aligned}
$$




$$
C_{1}=C_{2} \sqrt{\frac{v_{1}}{v_{2}}}
$$

For simplicity, the Poisson's ratio terms $v_{12}$ and $v_{21}$ were shortened to $v_{1}$ and $v_{2}$ respectively. If the Poisson's ratios are unknown, the ratio $v_{1} / v_{2}$ can be found using the initial moduli in the 1 and 2 axes.

$$
\frac{v_{1}}{v_{2}}=\frac{A_{1}}{A_{2}}
$$

Third, for shear stress $\tau$ and shear strain $\gamma$ in the 1-2 plane, the bilinear stress-strain prediction is as follows:

$$
\begin{array}{ll}
\tau_{12}\left(\gamma_{12}\right)=A_{12} \gamma_{12} & \text { for } \quad \gamma_{1} \leq \frac{C_{2}}{2\left(A_{2}-B_{2}\right)} \sqrt{\frac{A_{2}}{G_{12}}} \\
\tau_{12}\left(\gamma_{12}\right)=B_{12} \gamma_{12}+C_{12} & \text { for } \quad \gamma_{1} \geq \frac{C_{2}}{2\left(A_{2}-B_{2}\right)} \sqrt{\frac{A_{2}}{G_{12}}}
\end{array}
$$

where

$$
\begin{gathered}
A_{12}=G_{12}, \\
B_{12}=G_{12} \frac{B_{2}}{A_{2}} \\
C_{12}=\frac{C_{2}}{2} \sqrt{\frac{G_{12}}{A_{2}}} .
\end{gathered}
$$

If the initial shear modulus $\mathrm{G}_{12}$ is unknown it can be estimated by means of the empirical relationship:

$$
G_{12}=\frac{A_{2} \sqrt{v_{1} / v_{2}}}{2\left(1+\sqrt{v_{1} v_{2}}\right)} .
$$


Poisson's ratio was not known for this material, nor was it solved for experimentally, therefore the previous value of $G_{12}=284,000$ psi was used.

Representative stress-strain curves for the 1 and 2 axes were collected from the tension test data. The 1 axis curve was then fit with a bilinear relationship, shown below,

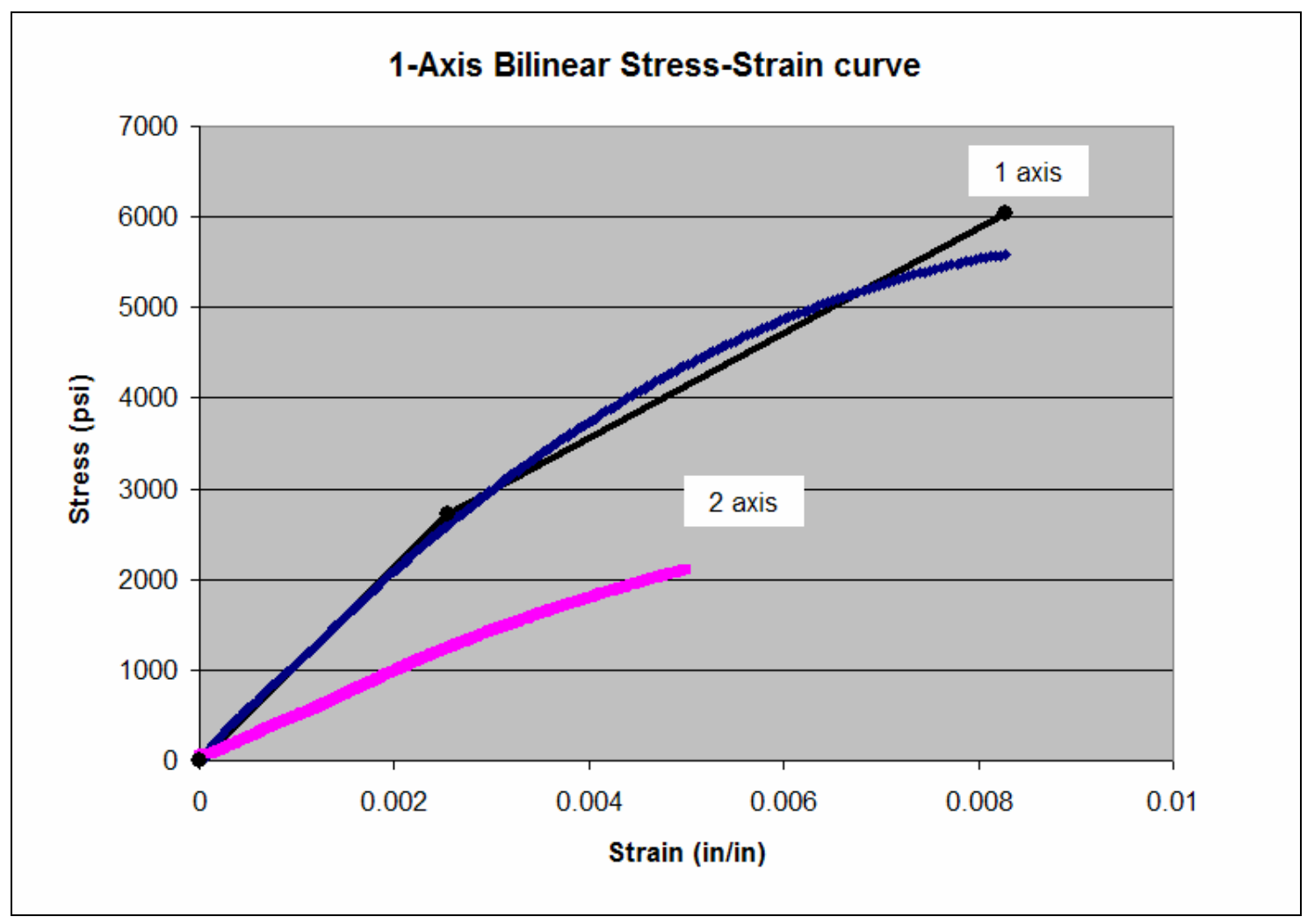

Figure FF: Fitting a Bilinear Curve to the 1-Axis

Then, the initial modulus of the 2 axis, $A_{2}=495,000$ psi, was used to predict the bilinear relationship for the 2 axis. Similarly, the initial shear modulus, $G_{12}=284,000$ psi, was used to predict a bilinear stress-strain relationship for the 1-2 plane. The results are shown on the next page in Figure GG. 


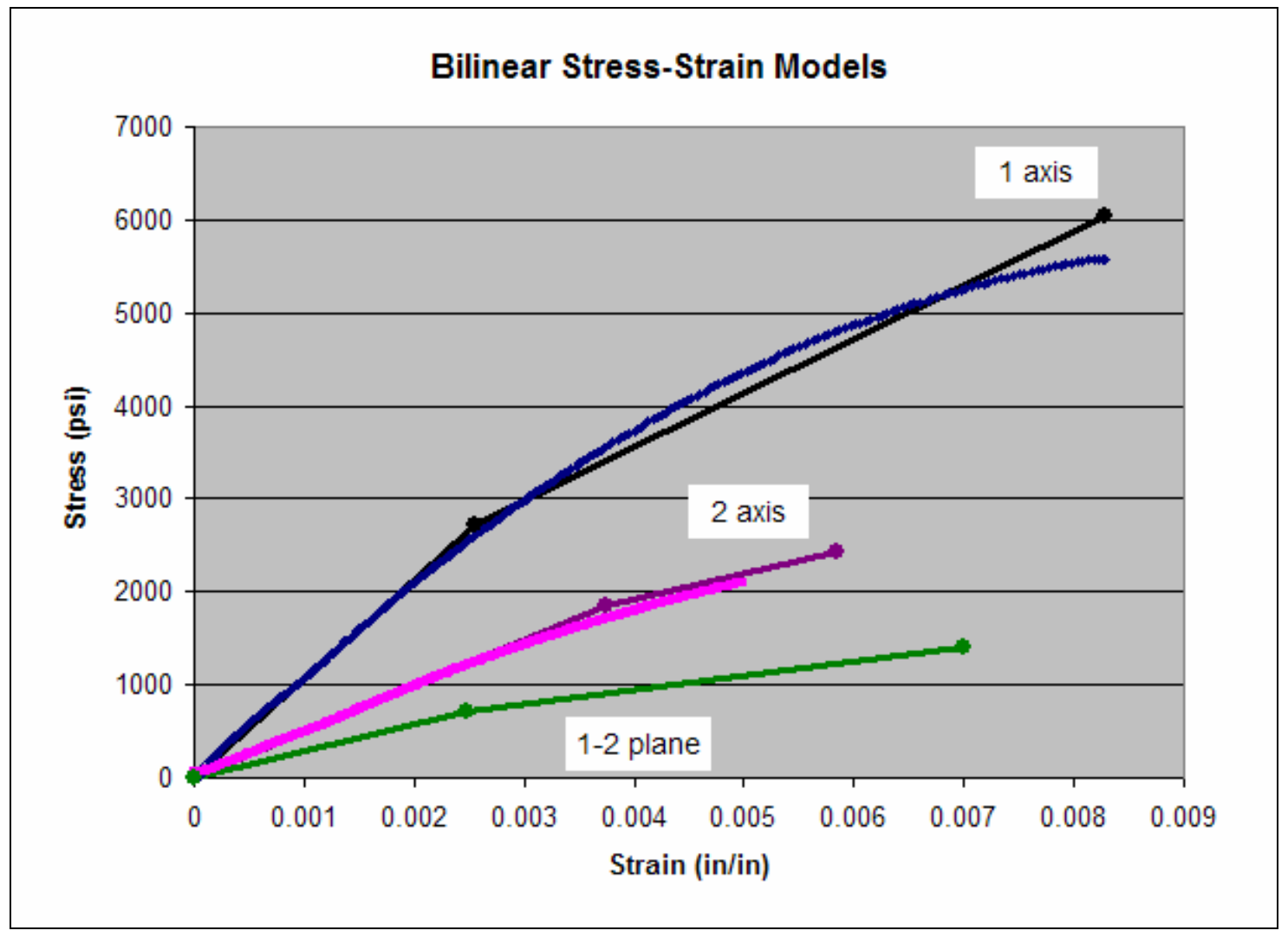

Figure GG: Bilinear Stress-Strain Predictions

This model seems to work well for this material. The nonlinear behavior on the 2 axis is fairly accurately captured by the bilinear prediction. However, the accuracy of the bilinear prediction for shear in the 1-2 plane cannot be assessed due to the lack of shear data past the linear-elastic region. If, however, future data can be found for nonlinear shear response, then this model can be readily applied to such data.

\subsection{New Constitutive Model}

The proposed constitutive model is a combination of both wood-based models as it predicts the bilinear stress-strain relationship at any orientation to the strong axis, given 
a nonlinear stress-strain relationship in the strong axis and the Poisson's ratios or the linear-elastic modulus at $90^{\circ}$ to the strong axis.

A series of simulated off-axis tension tests, under the bilinear stress-strain model, were performed in ANSYS to create a continuous set of data for the linear-elastic modulus $A$, nonlinear modulus $B$, and bilinear point strain $\varepsilon_{b}$. This set of data was compared to the physical experimental data. New equations for $A, B$ and $\varepsilon_{b}$ were developed to include angle to the strong axis $\theta$. These equations were based on a modified version of Hankinson's formula shown in Eq. 18 below.

$$
E_{\theta}=\frac{E_{1} E_{2}}{E_{1} \sin ^{4} \theta+\alpha\left(E_{1}+E_{2}\right) \sin ^{2} \theta \cos ^{2} \theta+E_{2} \cos ^{4} \theta} .
$$

This new formula was created to include the variable $\alpha$, which would make it possible to better fit the $\varepsilon_{b}$ data. It is also important to note that when $\alpha=1$, Eq. 18 reduces to Hankinson's formula. Eq. 18 can be further modified by relating $E_{2}$ to $E_{1}$ or $\varepsilon_{b 2}$ to $\varepsilon_{b 1}$ using the ratio $v_{2} / v_{1}$, see Eq. 19 below.

$$
E_{\theta}=\frac{E_{1}\left(\frac{v_{2}}{v_{1}}\right)}{\sin ^{4} \theta+\alpha\left(1+\frac{v_{2}}{v_{1}}\right) \sin ^{2} \theta \cos ^{2} \theta+\left(\frac{v_{2}}{v_{1}}\right) \cos ^{4} \theta},
$$

and

$$
\varepsilon_{b \theta}=\frac{\varepsilon_{b 1} \sqrt{\frac{v_{1}}{v_{2}}}}{\sin ^{4} \theta+\alpha\left(1+\sqrt{\frac{v_{1}}{v_{2}}}\right) \sin ^{2} \theta \cos ^{2} \theta+\sqrt{\frac{v_{1}}{v_{2}}} \cos ^{4} \theta} .
$$


Because the bilinear point strain is smaller at $0^{\circ}$ and larger at $90^{\circ}$, see Figure $\mathrm{JJ}$, but still linked through the Poisson's ratios, it uses $v_{1} / v_{2}$ instead of $v_{2} / v_{1}$. Using this form of the equation, both the $A$ and $B$ data could be matched well when $\alpha \approx 1$ and the $\varepsilon_{b}$ data could be matched well when $\alpha \approx 2$. The $A, B$, and $\varepsilon_{b}$ data along with their corresponding forms of Eq. 19 are shown in Figures HH through JJ.

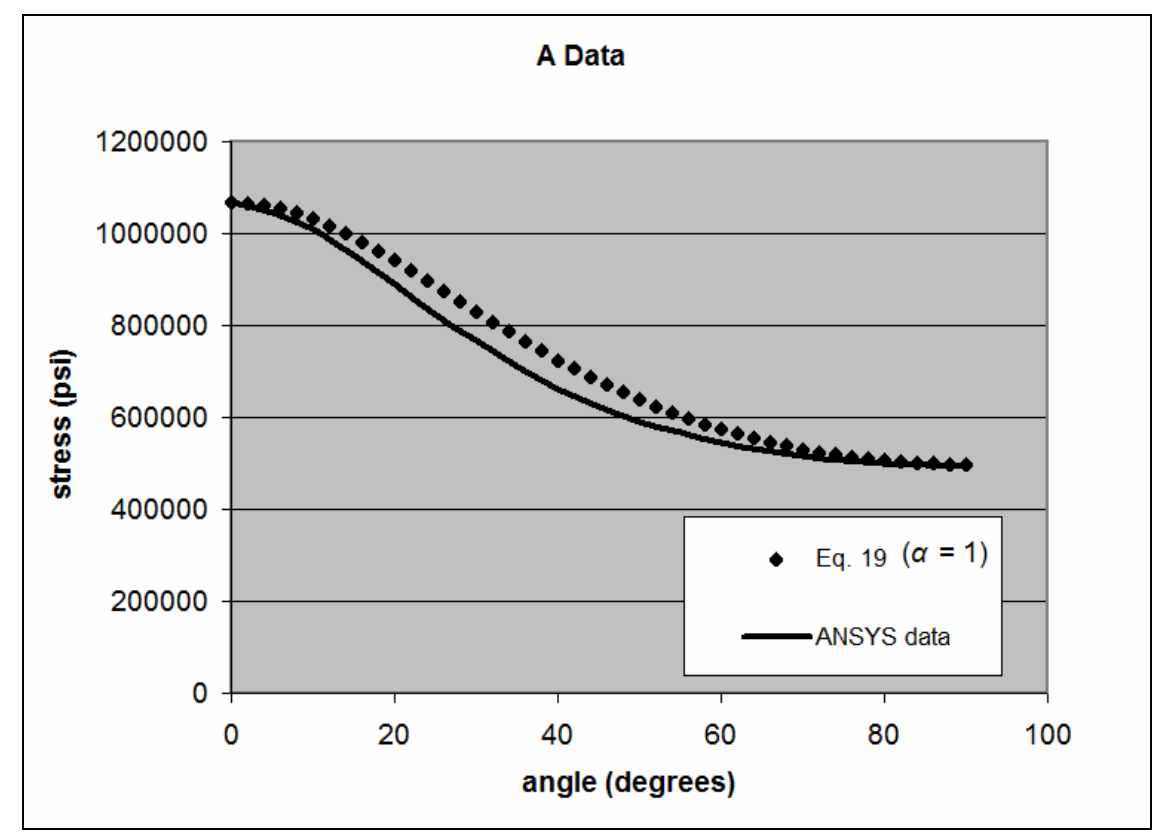

Figure HH: Matching $A$ Data 


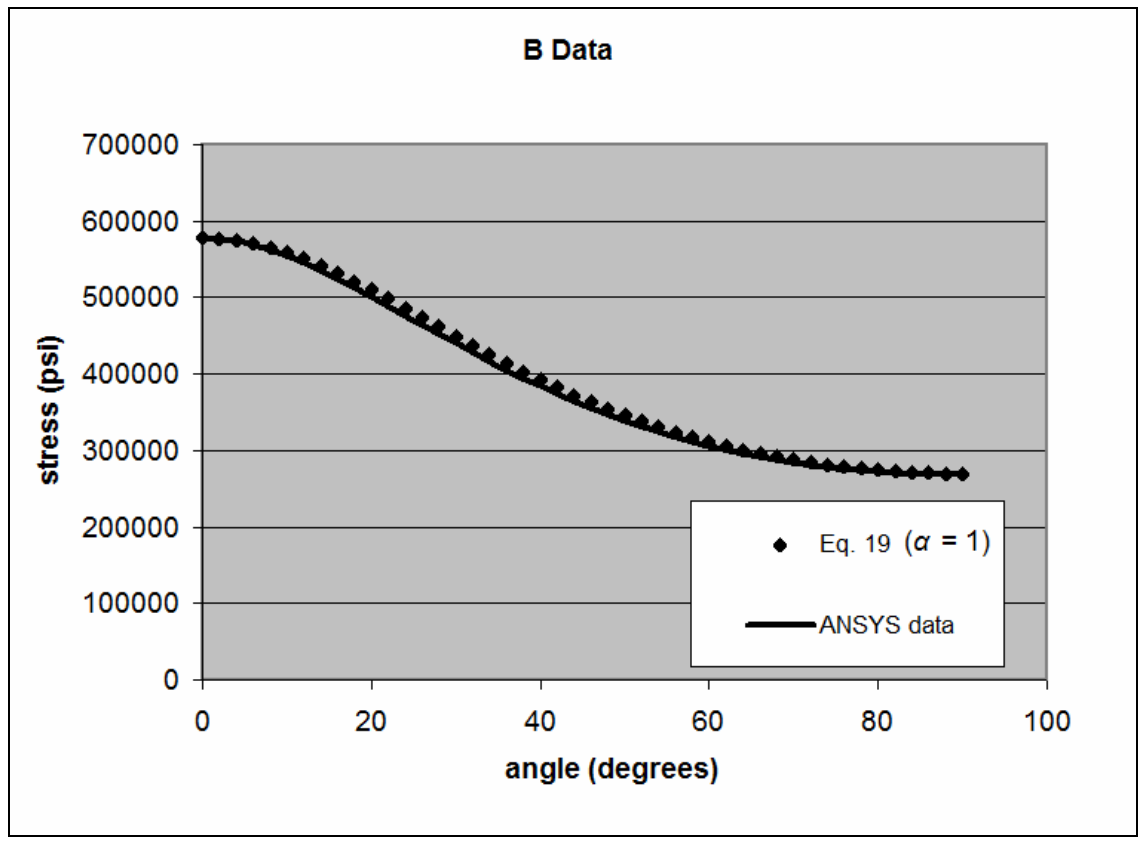

Figure II: Matching $B$ Data

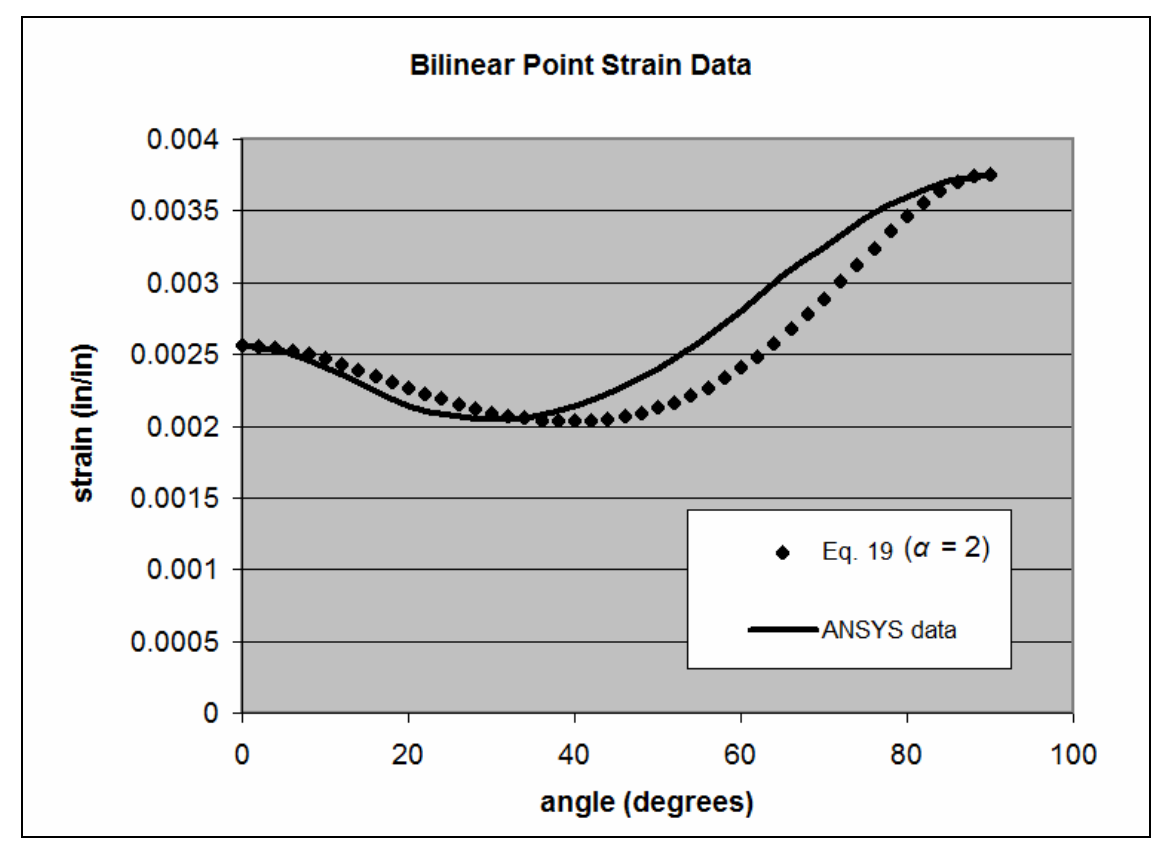

Figure JJ: Matching $\varepsilon_{b}$ Data 
Through Eq. 19, the bilinear model can consider any orientation between the $0^{\circ}$ and $90^{\circ}$ axes. A summary of the new constitutive relationship is given in Eq.'s 20 and 21 following.

$$
\begin{array}{cc}
\sigma_{\theta}\left(\varepsilon_{\theta}\right)=A_{\theta} \varepsilon_{\theta} & \text { for } \quad \varepsilon_{\theta} \leq \varepsilon_{b}, \\
\sigma_{\theta}\left(\varepsilon_{\theta}\right)=B_{\theta} \varepsilon_{\theta}+C_{\theta} & \text { for } \quad \varepsilon_{\theta} \leq \varepsilon_{b},
\end{array}
$$

where

$$
\begin{aligned}
& A_{\theta}=\frac{A_{1}\left(\frac{v_{2}}{v_{1}}\right)}{\sin ^{4} \theta+\left(1+\frac{v_{2}}{v_{1}}\right) \sin ^{2} \theta \cos ^{2} \theta+\left(\frac{v_{2}}{v_{1}}\right) \cos ^{4} \theta}, \\
& B_{\theta}=\frac{B_{1}\left(\frac{v_{2}}{v_{1}}\right)}{\sin ^{4} \theta+\left(1+\frac{v_{2}}{v_{1}}\right) \sin ^{2} \theta \cos ^{2} \theta+\left(\frac{v_{2}}{v_{1}}\right) \cos ^{4} \theta}, \\
& \varepsilon_{b \theta}=\frac{\varepsilon_{b 1} \sqrt{\frac{v_{1}}{v_{2}}}}{\sin ^{4} \theta+2\left(1+\sqrt{\frac{v_{1}}{v_{2}}}\right) \sin ^{2} \theta \cos ^{2} \theta+\sqrt{\frac{v_{1}}{v_{2}}} \cos ^{4} \theta}, \\
& C_{\theta}=\varepsilon_{b \theta}\left(A_{\theta}-B_{\theta}\right),
\end{aligned}
$$

and

$$
\frac{v_{2}}{v_{1}}=\frac{A_{2}}{A_{1}} .
$$

To demonstrate the effectiveness of this model, bilinear stress-strain curves were predicted for orientations of $33^{\circ}, 45^{\circ}$ and $68^{\circ}$ to the strong axis. These bilinear 
predictions were then compared to the experimental stress-strain data and can be seen in Figures KK through MM following.

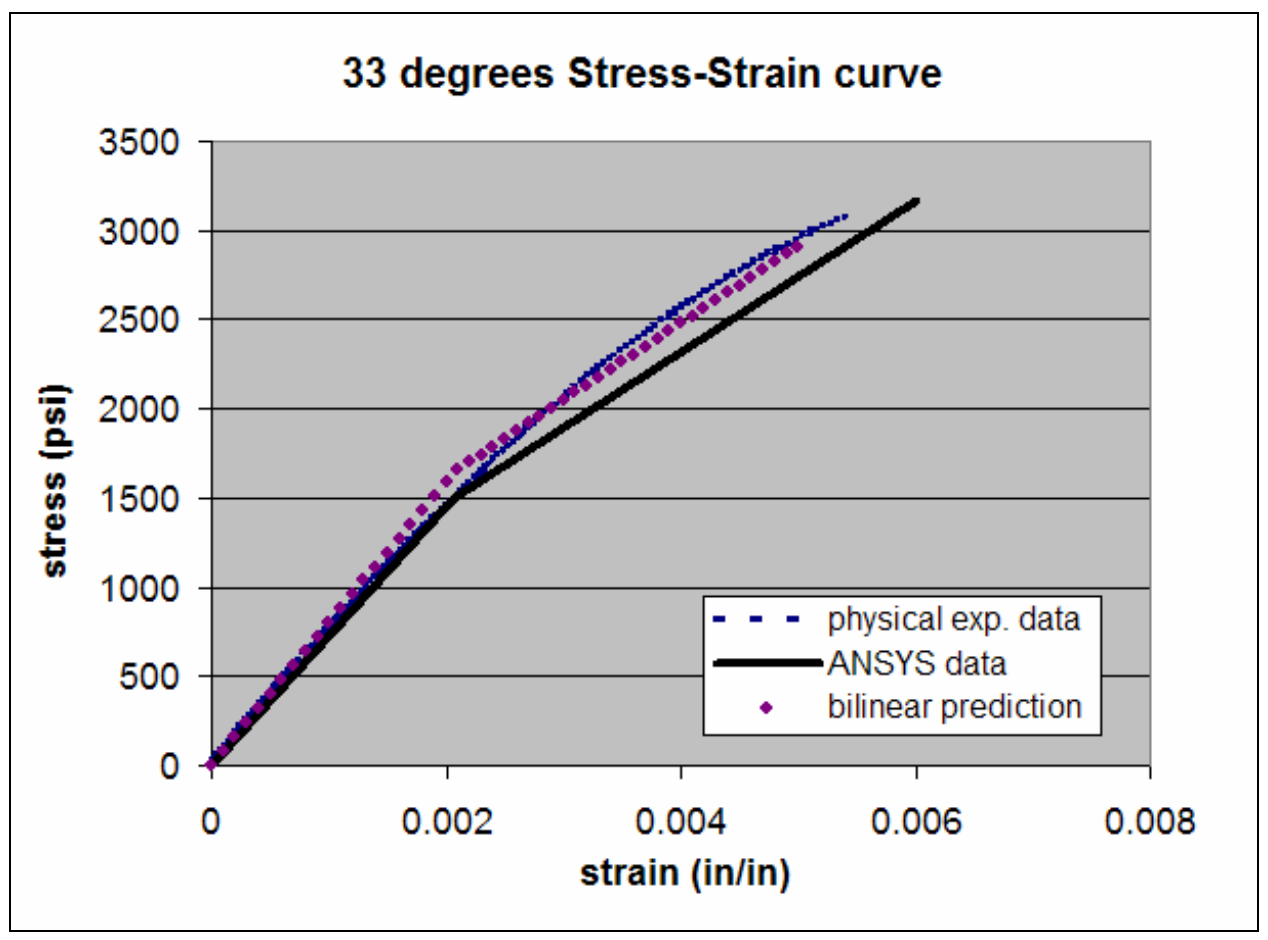

Figure KK: Bilinear Prediction for $33^{\circ}$ Data 


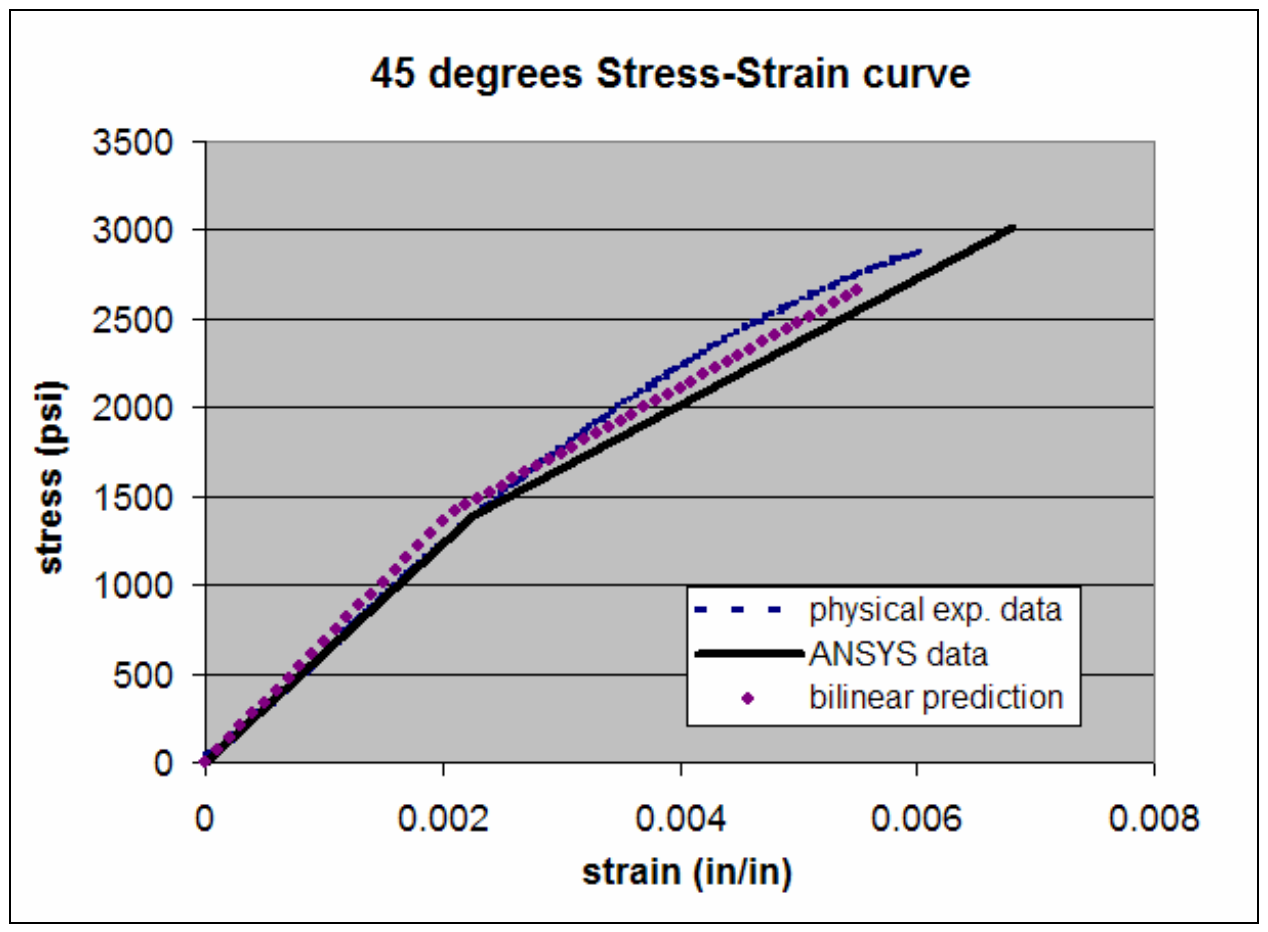

Figure LL: Bilinear Prediction for $45^{\circ}$ Data

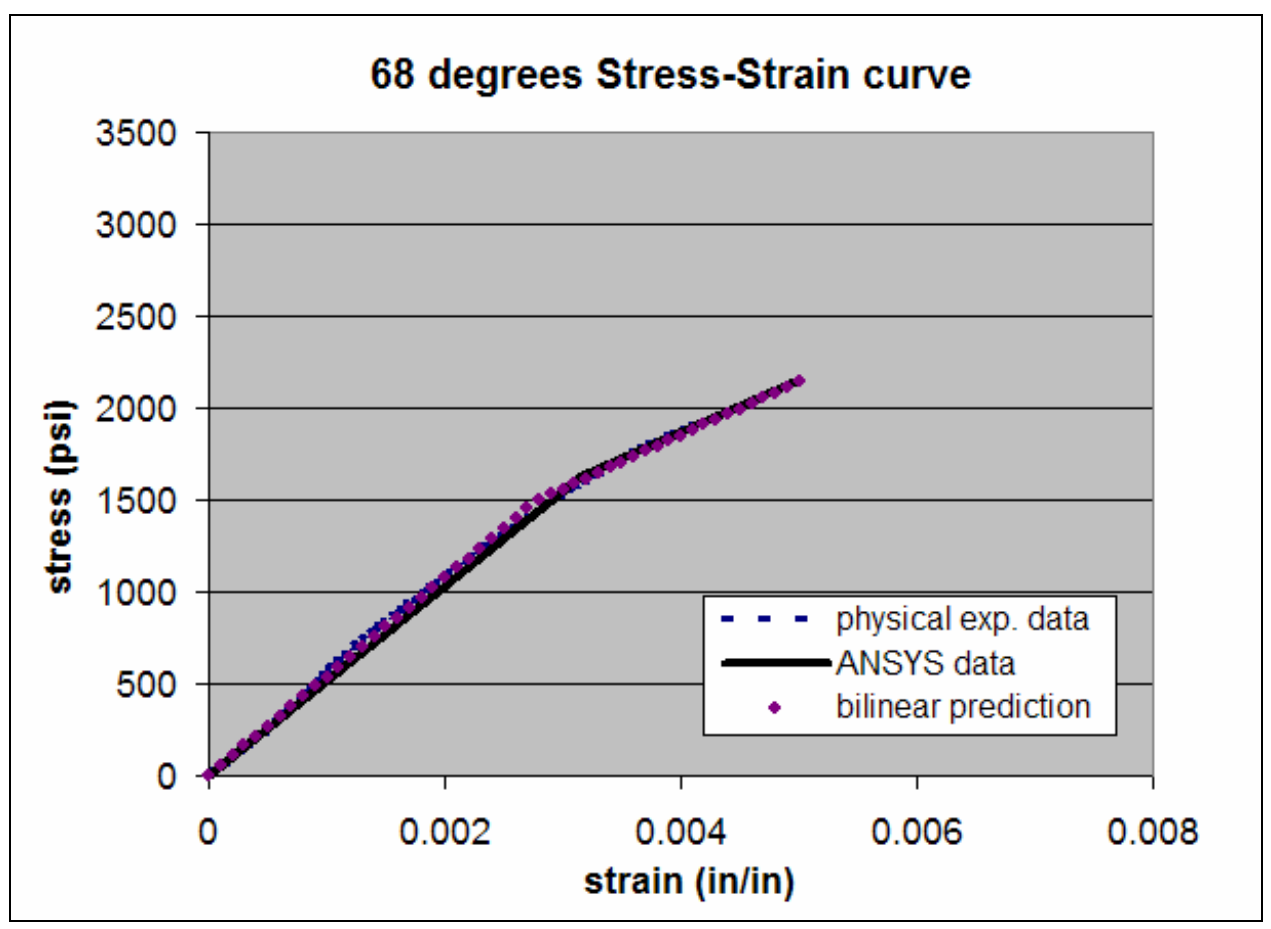

Figure MM: Bilinear Prediction for $68^{\circ}$ Data 
The previous figures show that this proposed constitutive model accurately captures the stress-strain relationships for any orientation to the strong axis. A summary plot of the bilinear constitutive model with the experimental data is shown in Figure NN.

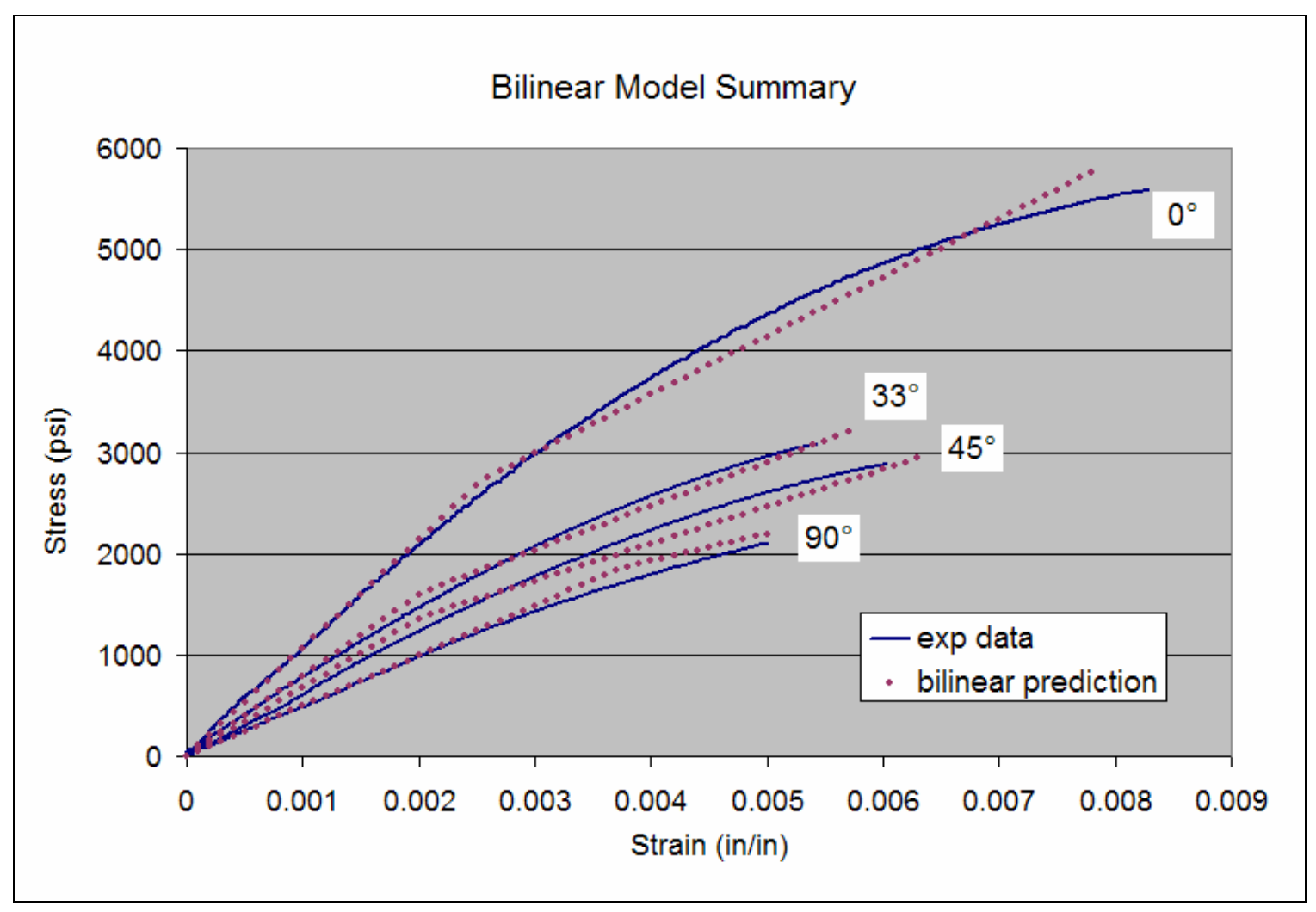

Figure NN: Summary of Bilinear Stress-Strain Predictions

The plot above shows a comparison of bilinear stress-strain predictions for $0^{\circ}, 33^{\circ}, 45^{\circ}$ and $90^{\circ}$. The $68^{\circ}$ orientation is not included in the summary plot for visual clarity. As expected, the bilinear predictions for angles between $0^{\circ}$ and $90^{\circ}$ are enveloped by the bilinear curves of each. 


\subsection{APPLICATION IN FINITE ELEMENT ANALYSIS}

The bilinear constitutive model was used to perform a nonlinear finite element analysis (FEA) using the computer program ANSYS. A one-story lateral load resisting frame with a distributed load at the roof level was analyzed.

\subsection{Computer Modeling}

Plane elements with a thickness of 0.5 inches were used to model all parts of the frame, resulting in a braced frame with moment connections. The frame elevation with dimensions is shown in figure OO below.

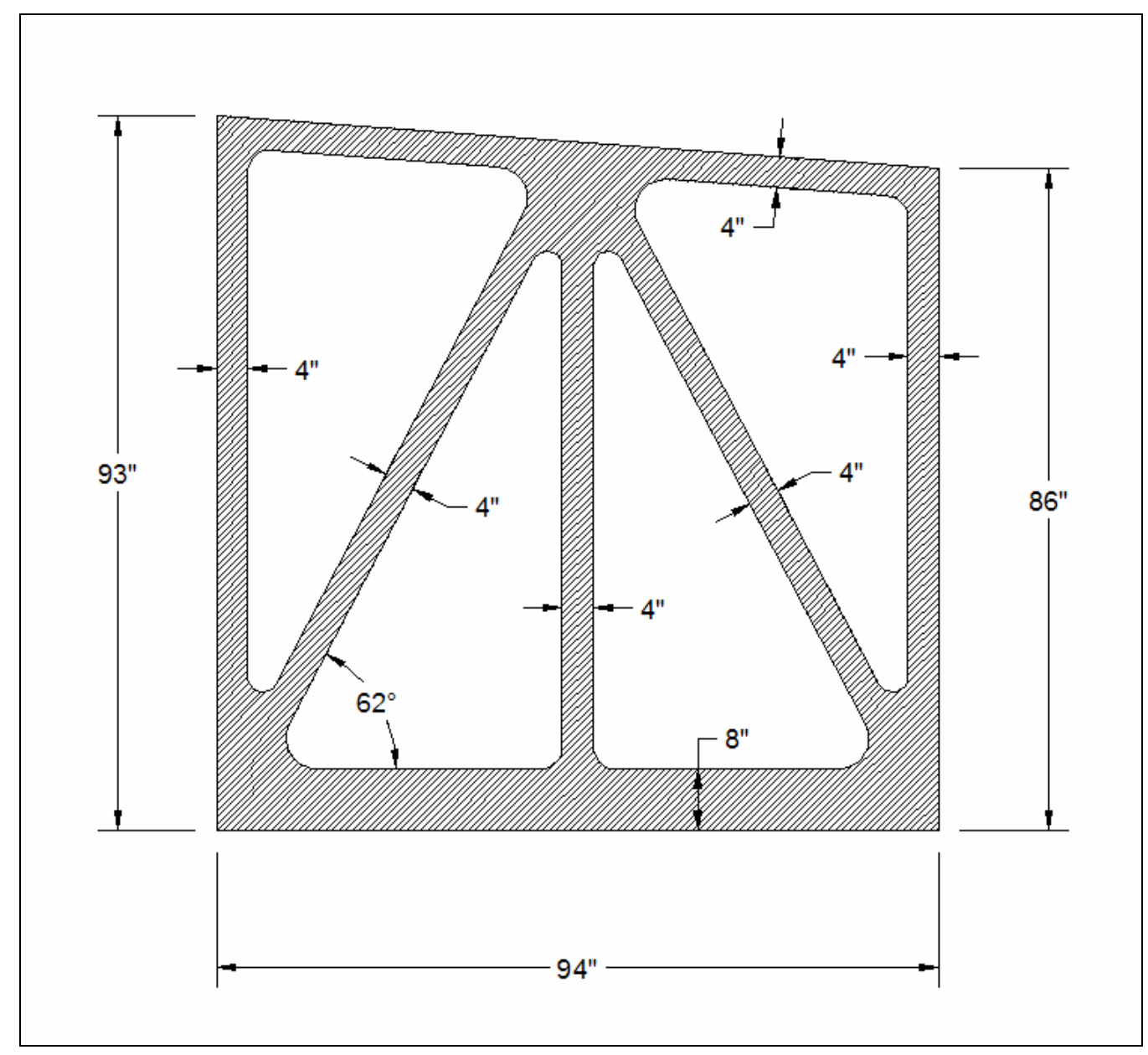

Figure 00: Analyzed Frame

Nonlinear Modeling of a Sustainable Material 
The linear-elastic material properties used in this simulation were defined as $E_{x}$ and $E_{y}$ for Young's modulus in the longitudinal and transverse directions, $G_{x y}$ for the shear modulus, and $v_{x y}$ for Poisson's ratio; the values of these constants were as follows:

$$
\begin{aligned}
& E_{x}=1,064,000 \mathrm{psi}, \\
& E_{y}=495,000 \mathrm{psi} \\
& G_{x y}=284,000 \mathrm{psi}, \text { and } \\
& v_{x y} \text { is estimated as } 0.1 .
\end{aligned}
$$

Generalized Anisotropic Hill Potential was used to simulate nonlinearity by defining yield stresses and post-yield moduli. Although this PP/kenaf material was not proven to yield, this nonlinear model still applied because plasticity was not an issue. The required constants included $\sigma_{\text {xyield }}$ and $\sigma_{\text {yyield }}$ for the yield stresses in the $x$ and $y$ directions, $\tau_{x y y i e l d}$ for the yield shear stress, and $E_{x p l a s t i c}, E_{y p l a s t i c}$ and $G_{x y p l a s t i c}$ for the corresponding post-yield moduli; the values of these constants were as follows:

$$
\begin{aligned}
& \sigma_{\text {xyield }}=2727 \mathrm{psi}, \\
& \sigma_{\text {yyield }}=1857 \mathrm{psi}, \\
& \tau_{\text {xyyield }}=703 \mathrm{psi}, \\
& E_{\text {xplastic }}=578,000 \mathrm{psi}, \\
& E_{\text {yplastic }}=268,000 \mathrm{psi}, \text { and } \\
& G_{\text {xyplastic }}=154,000 \mathrm{psi} .
\end{aligned}
$$

The frame mesh is shown in figure PP on page 55 following. 


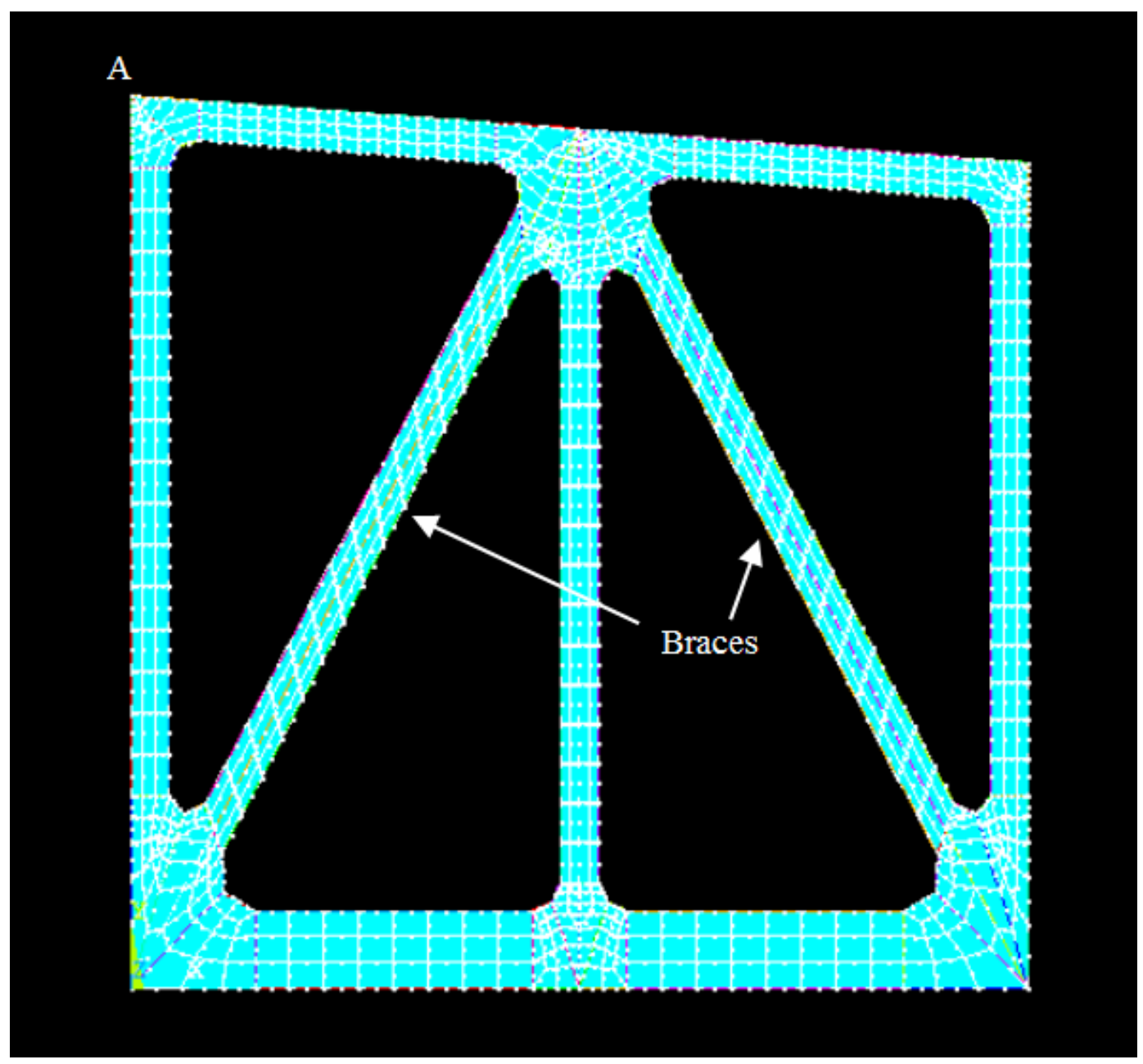

Figure PP: Frame Mesh

The element coordinates were adjusted to align the strong axes along the lengths of the members, simulating fiber alignment from extrusion or injection molding. Therefore, the strong axes of the braces are in the same orientation as the braces themselves. It was modeled this way because injection molding is one possible construction solution using this material. Another construction solution would be to take one large sheet of extruded material and cut out pieces leaving one continuous frame. The strong axis would then be aligned at all points on the frame including the braces. This would have resulted in the 
braces having a lower Young's modulus and failure load. A pushover analysis would have shown decreased frame stiffness and a decreased ultimate load and deflection. The simulated fiber alignment can be seen in figure QQ below.

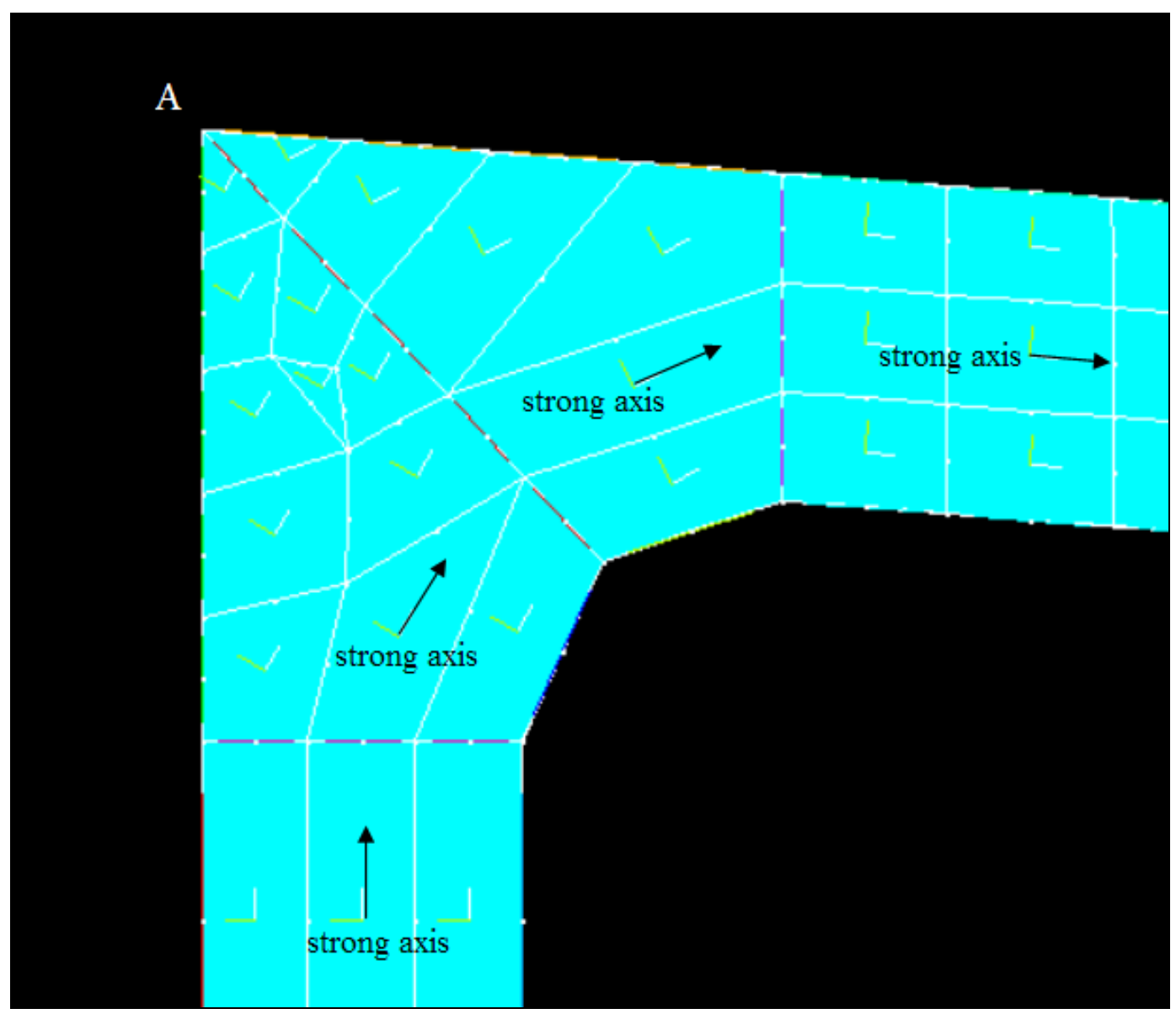

Figure QQ: Close-up of Element Local Axes

Lateral load was placed at the roof level and horizontal deflections were monitored at point A. The frame was pushed until the stress in the braces reached $4650 \mathrm{psi}$, the average failure stress at $0^{\circ}$ from the tension tests; see Table 2 on page 21 . A force displacement relationship was recorded. 


\subsection{Analysis Results}

A simple hand calculation using the bilinear-stress strain prediction shows the effectiveness of this model in both hand and finite element analyses. The forcedisplacement relationship for both is shown below in figure RR.

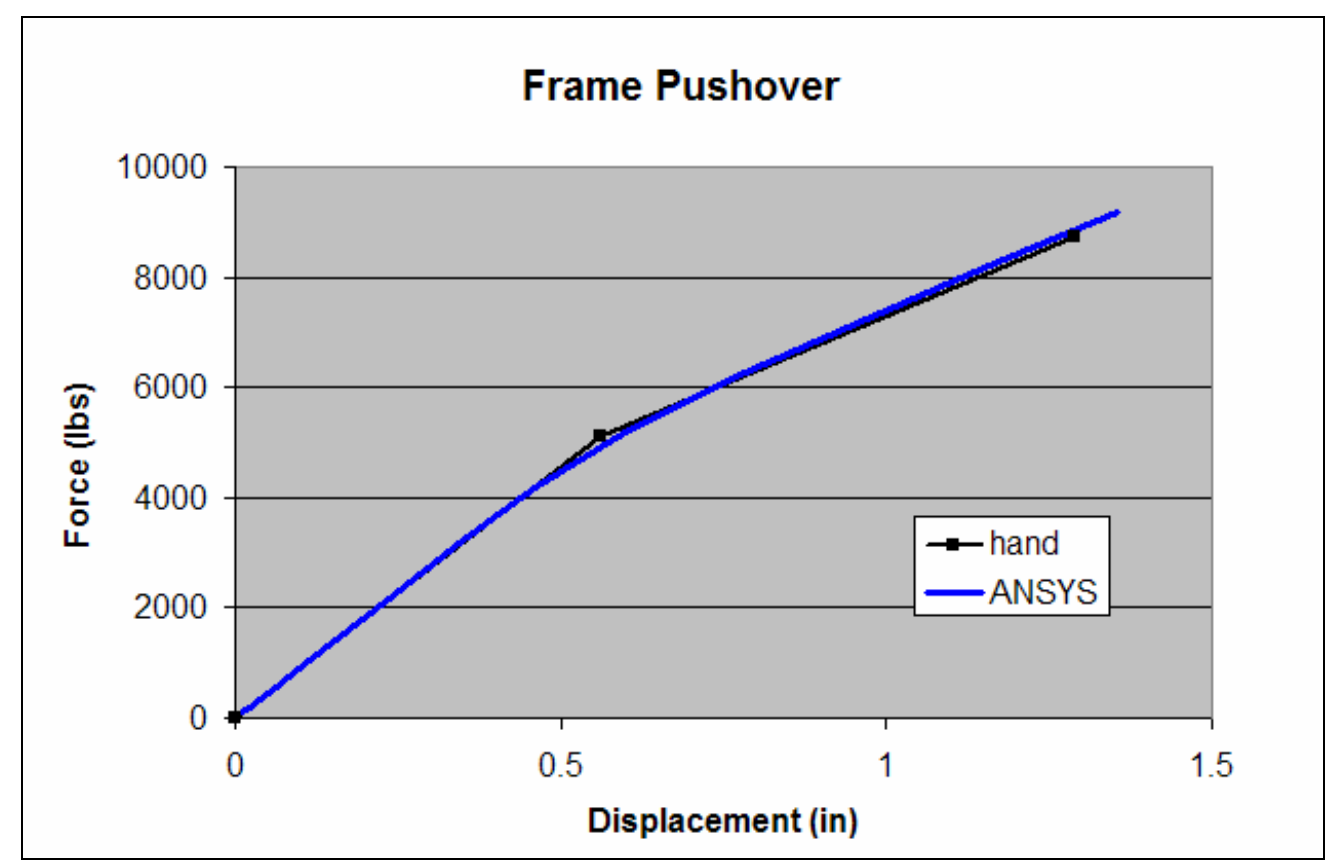

Figure RR: Force-Displacement Relationship for the Analyzed Frame

Both models predict very similar pushover curves and demonstrate the effectiveness of the proposed constitutive model in nonlinear analyses of this plastic-fiber composite. 


\subsection{CONCLUSIONS}

Experimental work was done for Young's modulus at varying orientations $E_{\theta}$ and for the shear modulus $G$. Physical experimental results were verified through simulated experiments using ANSYS. The experimental testing done for the shear modulus gives evidence that this PP/kenaf material has noticeably different properties in the two weak axes which is contrary to the initial assumptions. However, there was difficulty encountered when trying to relate the shear stress $\tau$ to the shear strain $\gamma$ past yield. New experimental data is required to complete the linkage between $E_{\theta}$ and G. Therefore this study focused on a nonlinear constitutive model of $E$.

A semi-empirical approach was used to create a new constitutive stress-strain model that has been demonstrated to predict the nonlinear stress-strain response of this material at varying orientations to the strong axis. An important strength of this model is the application of simple bilinear curves to distinctly nonlinear stress-strain data. This simplification is demonstrated through hand analyses of load resisting systems.

The second strength of this model is that it relates the apparent yield strains in all orientations to the strong axis. Bilinear approximations also assure distinct yield points, which is why ANSYS uses the bilinear model for orthotropic materials. Nonlinear finite element analyses of the larger emergency shelter were performed to demonstrate the effectiveness of this model when implemented into ANSYS.

The third strength of this model is that it captures the stress-strain response in any possible orientation, whereas previous methods only linked the three orthogonal axes. 
Weaknesses stem from the lack of shear data available, which is why this model is based on the empirical Hankinson's formula. Further testing may be done to see if a theoretical relationship, such as the classic orthotropic elasticity equation, can be used to derive a model similar to the one proposed here. This constitutive model has been shown to work well for this PP/kenaf material; however, further research may also be done to investigate the effectiveness of this model with other orthotropic materials such as wood. 


\subsection{REFERENCES}

(Bindzi and Samson 1995) Bindzi, Isaac, and Marcel Samson. "New Formula for Influence of Spiral Grain on Bending Stiffness of Wooden Beams." J of Structural Engr November (1995): 1541-302.

(Carpenter 2009) Carpenter, Christine. "Effects of Accelerated Weathering on the Flexural Properties of Kenaf-Reinforced Polypropylene Composites." California Polytechnic State University, Senior Project, instructor: Saliklis, Edmond P. (2009).

(Jalali and Taheri 1999) Jalali, S. Javad, and Farid Taheri. "A New Test Method for Measuring the Longitudinal and Shear Moduli of Fiber Reinforced Composites." J of Composite Materials 33.23 (1999): 2134-2160.

(Johnson and Urbanik 1984) Johnson, M.W. Jr., and T.J. Urbanik. "A Nonlinear Theory for Elastic Plates With Application to Characterizing Paper Properties." J of Applied Mech 106 (1984): 146-152.

(Jones 1975) Jones, R. M. Mechanics of composite materials. McGraw-Hill, New York.

(Lee 2007) Lee, Erik. "Plastic/Wood Strength Test Experiment." California Polytechnic State University, instructor: Saliklis, Edmond P. (Summer 2007).

(Raj and Kokta 1991) Raj, R. G., and B. V. Kotka. "Reinforcing High Density Polyethylene With Cellulosic Fibers. I: The Effect of Additives on Fiber Dispersion and Mechanical Properties." Polymer Engr and Science September (1991) 31: 146-152.

(Rosen 1972) Rosen, B. Walter. “A Simple Procedure for Experimental Determination of the Longitudinal Shear Modulus of Unidirectional Composites." J of Composite Materials October (1972) 6: 552-554.

(Saliklis, Urbanik and Tokyay 2003) Saliklis, Edmond P., T.J. Urbanik, and B. Tokyay. "Bilinear Modelling of Cellulosic Orthotropic Nonlinear Materials." J of Pulp and Paper Science 29.12 (2003): 407-411.

(Saliklis and Falk 2000) Saliklis, Edmond P., and Robert H. Falk. "Correlating Off-Axis Tension Tests to Shear Modulus of Wood-Based Panels." J of Structural Engr May (2000): 621-625. 
(Saliklis and Arens 2009) Saliklis, Edmond P., and Robert M. Arens. "Rapidly assembled emergency shelters made from 'green' materials." Proceedings of the Int'l Association for Shell and Spatial Structures Symposium (IASS), Valencia. September 28 to October 7, 2009. Universidad Politecnica de Valencia, Spain.

(Shih and Lee 1978) Shih, C.F., and D. Lee. "Further Developments in Anisotropic Plasticity.” J Engin Materials Technol Am Soc Mech Engin 100 (1978): 294-302.

(Yoshihara 1998) Yoshihara, Hiroshi, et al. "Measurement of the shear modulus of wood by static bending tests." The Japan Wood Research Society 44 (1998): 15-20.

(Yoshihara 1999) Yoshihara, Hiroshi, et al. "Applicability of the Iosipescu shear test on the measurement of the shear properties of wood." The Japan Wood Research Society 45 (1999): 24-29.

(Yoshihara 2002) Yoshihara, Hiroshi, et al. "Measurement of the shear modulus of wood by asymmetric four-point bending tests." The Japan Wood Research Society 48 (2002): 14-19. 


\subsection{APPENDIX}

Four-Point Bending Test Displacement Check

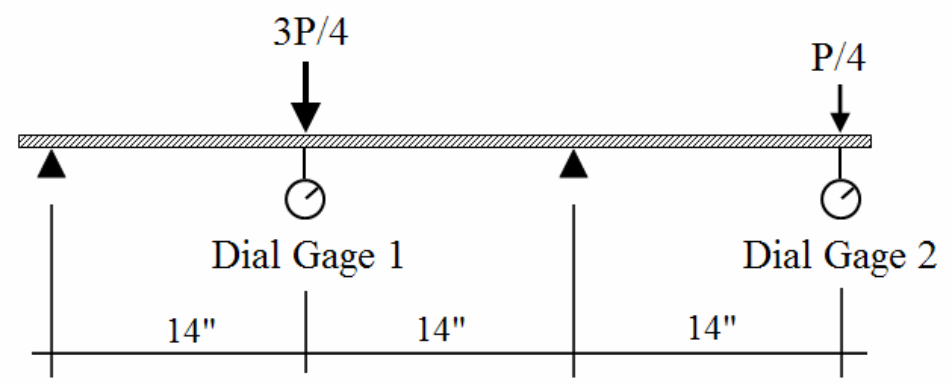

Check displacements at a load of $300 \mathrm{lbs}$

Machine Reading $=0.210$ ”

Dial 1 Reading $=0.214 "$

Dial 2 Reading $=0.225 "$

$\%$ error between dials $=(0.225-0.215 ") / 0.225 " \mathrm{x} 100=4.89 \%$ error $\leftarrow$

Predicted displacement from dials (through geometry) $=0.217$ "

$\%$ error between predicted and machine $=$

$$
(0.217-0.210 ") / 0.217 \text { " x } 100=3.11 \% \text { error } \leftarrow
$$

Check displacement at a load of $900 \mathrm{lbs}$

Machine Reading $=0.663 "$

Dial 1 Reading $=0.681^{\prime}$

Dial 2 Reading $=0.715^{\prime}$

$\%$ error between dials $=(0.715-0.681 ") / 0.715 "$ x $100=4.76 \%$ error $\leftarrow$

Predicted displacement from dials (through geometry) $=0.690$ ”

$\%$ error between predicted and machine $=$

$$
(0.690-0.663 ") / 0.690 " \text { x } 100=3.84 \% \text { error } \leftarrow
$$


Frame Pushover Hand Calculation:

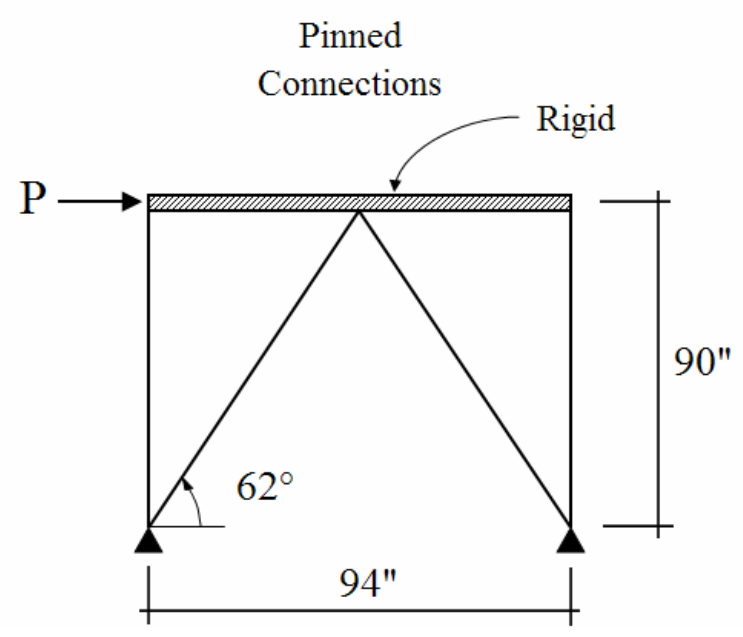

Given:

$$
\begin{aligned}
& A_{\text {brace }}=0.5 " \times 4 "=2 \mathrm{in}^{2} \\
& \mathrm{~L}_{\text {brace }}=\sqrt{ }\left(47^{2}+90^{2}\right)=102 " \\
& \mathrm{E}_{\text {elastic }}=1064000 \mathrm{psi} \\
& \mathrm{E}_{\text {plastic }}=578000 \mathrm{psi} \\
& \sigma_{\text {yield }}=2720 \mathrm{psi} \\
& \sigma_{\text {fail }}=4650 \mathrm{psi}
\end{aligned}
$$

Solution:

$$
\begin{aligned}
& \mathrm{K}_{\text {before yield }}=(2)\left(2 \mathrm{in}^{2}\right)(1064000 \mathrm{psi}) \cos ^{2}(62) /(102 ”)=9196 \mathrm{lb} / \mathrm{in} \\
& \mathrm{F}_{\text {brace yield }}=(2720 \mathrm{psi})\left(2 \mathrm{in}^{2}\right)=5440 \mathrm{lbs} \\
& \mathrm{P}_{\text {yield }}=(2)(5440 \mathrm{lbs}) \cos (62)=5108 \mathrm{lbs} \leftarrow \\
& \Delta_{\text {yield }}=(5108 \mathrm{lb}) /(9196 \mathrm{lb} / \mathrm{in})=0.555^{\prime \prime} \leftarrow
\end{aligned}
$$

$\mathrm{K}_{\mathrm{after} \text { yield }}=(2)\left(2 \mathrm{in}^{2}\right)(578000 \mathrm{psi}) \cos ^{2}(62) /(102 ”)=4996 \mathrm{lb} / \mathrm{in}$

$\mathrm{F}_{\text {brace fail }}=(4650 \mathrm{psi})\left(2 \mathrm{in}^{2}\right)=9300 \mathrm{lbs}$

$\mathrm{P}_{\text {fail }}=(2)(9300 \mathrm{lbs}) \cos (62)=8732 \mathrm{lbs} \leftarrow$

$$
\Delta_{\text {fail }}=0.555^{\prime \prime}+(8732-5108 \mathrm{lbs}) /(4947 \mathrm{lb} / \mathrm{in})=1.288^{\prime} \quad \leftarrow
$$




\subsection{GLOSSARY}

bilinear point - the point at which the stress-strain curve changes slope.

isotropic - material properties are the same in every direction.

linear-elastic range - the range in which the maximum strain is less than the bilinear point strain for any portion of a specimen.

nonlinear modulus - the slope of the stress-strain curve past the bilinear point.

nonlinear range - the range in which the minimum strain is more than the bilinear point strain for any portion of a specimen.

modulus of rupture - the normal stress on the tension side of a specimen loaded in bending, when the material starts to yield.

orthotropic - material properties depend on the direction in which they are measured. 


\subsection{ACRONYMS}

PP

HDPE high-density polyethylene

FEA finite element analysis

ASTM American Society for Testing and Materials

NDS National Design Specification

UTM universal testing machine 